\title{
An Undetectable Work-Ethic: \\ Design Activism's detour into subcultures \\ of queer disability and innovation.
}

by

Juan Saavedra

A thesis submitted to the Faculty of Graduate and Postdoctoral Affairs in partial fulfillment of the requirements for the degree of

Master of Design

in

Industrial Design

Carleton University

Ottawa, Ontario

(C) 2019, Juan Saavedra 


\begin{abstract}
LGBTQ communities globally continue to gain momentum in social acceptance yet continue to be challenged in their pursuits of equality. This research inquiry seeks to acknowledge and illuminate Design-Activism's relevancy among LGBTQ communities with a focus on Activism, Entrepreneurship and (Invisibility) Disability Culture. This study uses a mixed methods approach to understand Design-Activism's capacity towards accessing new and alternative modes of social inclusion. Drawing from theoretical and design-driven generated forms of knowledge, this study aligns pre-assumed disconnected concepts and highlights the unnoticed interconnectedness. Discovered are the boundaries and forces needed to expand Design's potential role towards championing Design beyond traditional commercial applications, with a concentrated effort towards service gaps and accessibility. These communities, although historically antagonized, demonstrate genuine insights and contributions towards the practice of Design Activism, thus revealing much more about people and the importance of Design.
\end{abstract}

Keywords: LGBTQ, Design Activism, Disability Knowledge, Access, Queer Theory 


\section{Acknowledgements}

This journey wasn't easy. Starting on a negative note never seems right, however it is the truth of this story, making this journey a lot more exciting than it needed to be. There are many thanks to give. First off, I wish to express gratitude for the support from my supervisors: Bjarki Hallgrimsson and Dr. Xuan Thuy Nguyen. Thank you Bjarki for reassuring my thesis belonged within the School of Industrial Design. Thank you, Dr. Nguyen, for highlighting that self-awareness continues to be one of my strongest traits. I am even more grateful to the many queer individuals who took the time to contribute to this study. Especially the radical queers and weirdos who helped make the Photo-Voice activity more interesting than I anticipated.

I'd also like to thank John Rich and Ehsan Mohseni, both of whom taught me lessons school could never teach. Having met both of you during my studies has been strange and beautiful; after all, there is no harmony without the harm.

Lastly, to any young or old queers or weirdos ever reading this, it never really gets better. Nonetheless, may you find the courage to change for the better, especially when you are constantly told change is impossible. Stay weird. And always be queer AF. 


\section{Table of Contents}

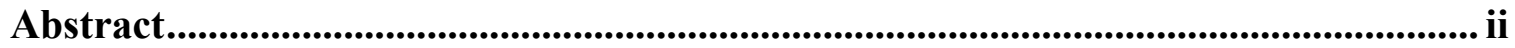

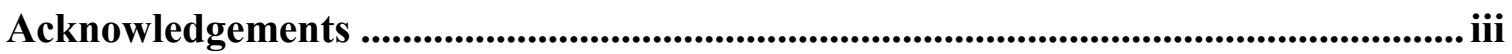

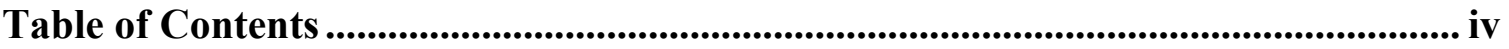

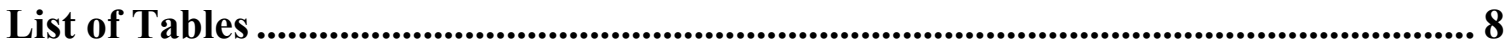

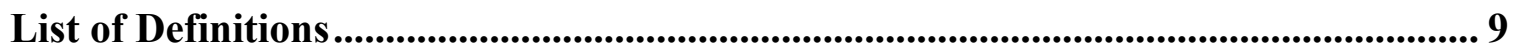

List of Figures, Graphs and Images ........................................................................... 12

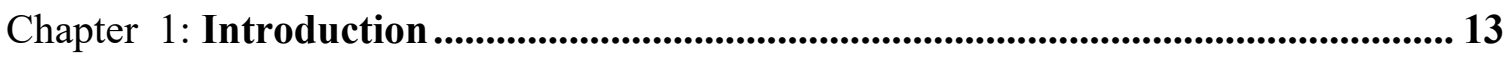

1.1 Background, Starting Point, and Original Motive ................................................ 13

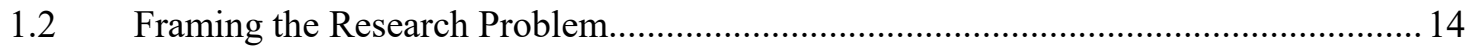

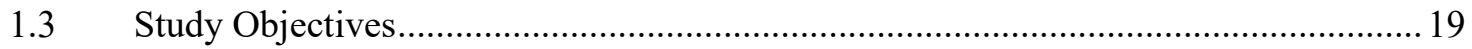

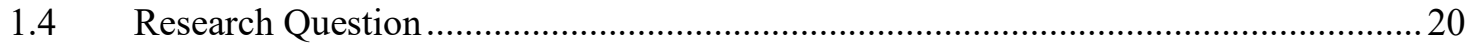

Chapter 2: Literature Review..................................................................................... 20

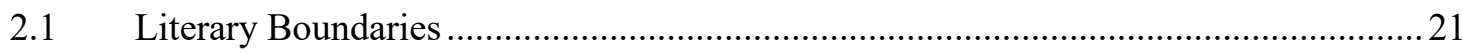

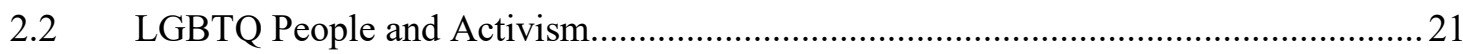

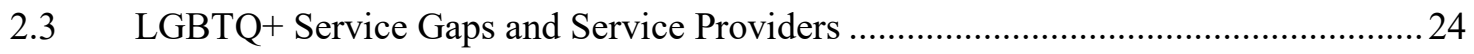

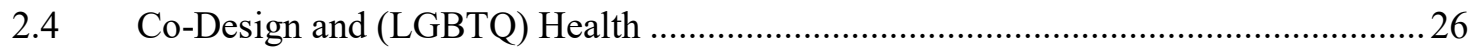

2.5 Consumers and Producers: Advertising-Activism, Services and HIV .......................27

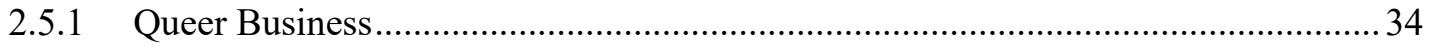

2.6 Entrepreneurship, Design and Social Innovation. ................................................ 36

2.6.1 Design and its relationship to Social Innovation.................................................... 36

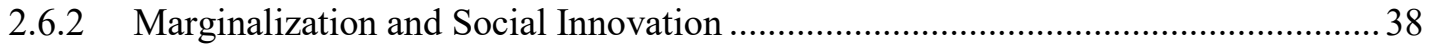

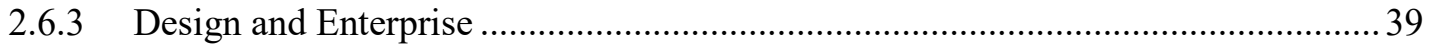

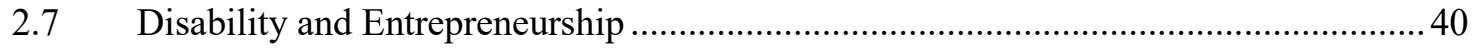




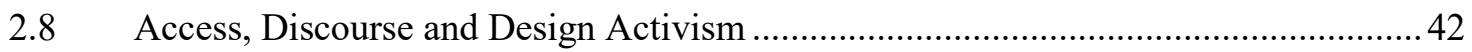

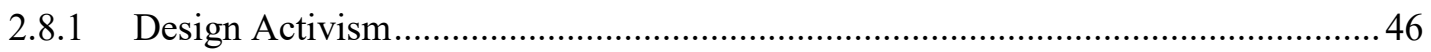

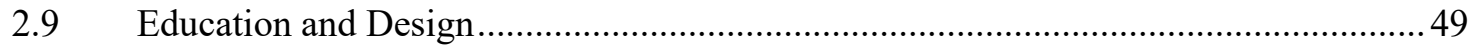

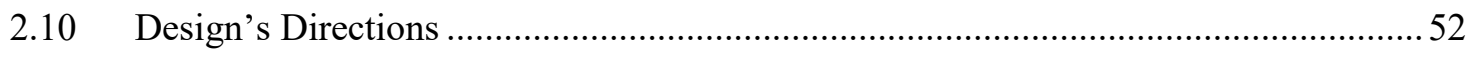

Chapter 3: Method ......................................................................................................... 54

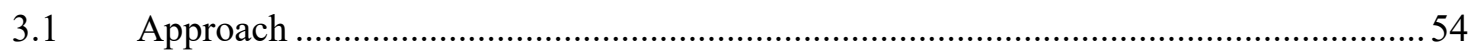

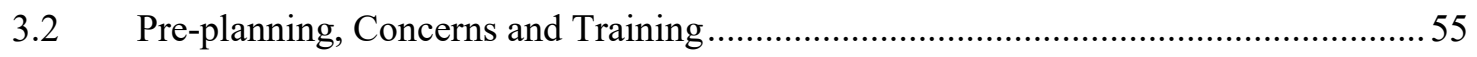

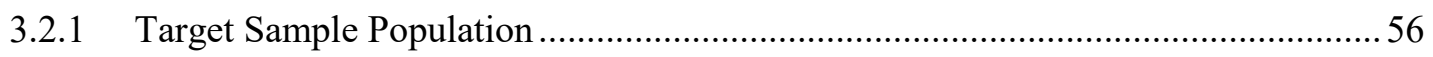

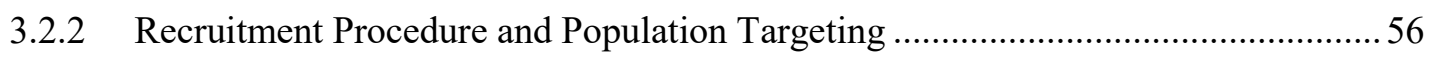

3.3 Method 1 - Terms and Definition Online Questionnaire Form ..................................58

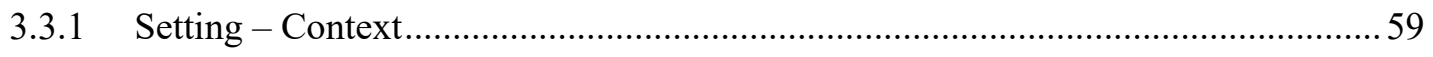

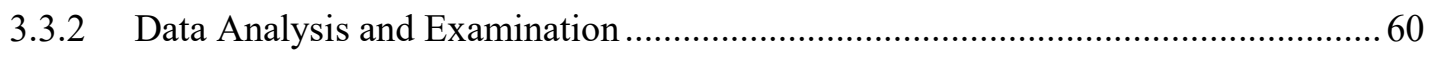

3.4 Method 2 - Card-Sorting and Semi-Structured Interviews ....................................... 60

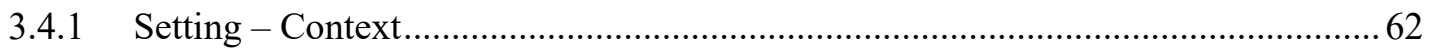

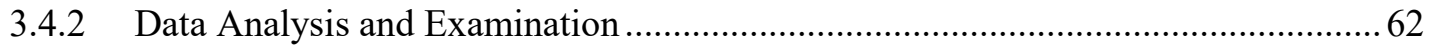

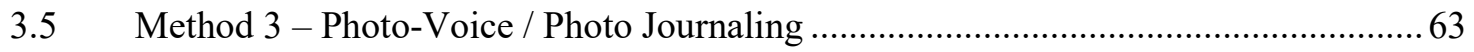

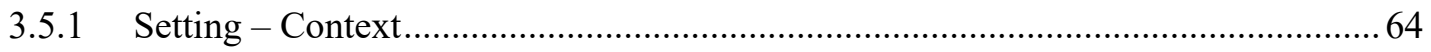

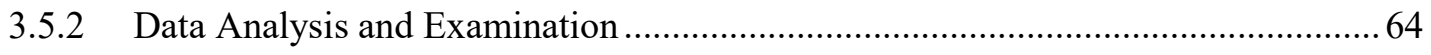

Chapter 4: Findings .................................................................................................................... 65

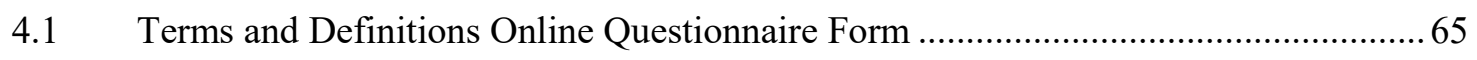

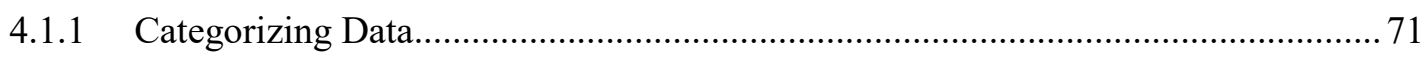

4.1.2 Comparing Service Users and Providers....................................................... 71

4.2 Card-Sorting and Semi-Structured Interviews ................................................... 72

4.2.1 Final Card-Sort of Service Providers …............................................................. 73

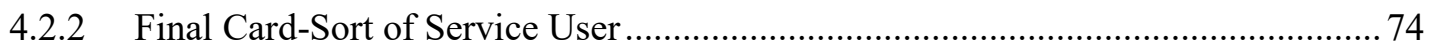


4.2.3 Semi-Structured Interviews - SWOT (Analysis) and Overview

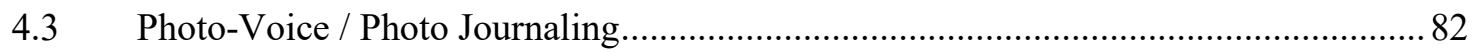

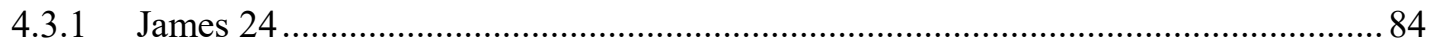

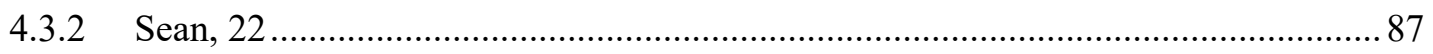

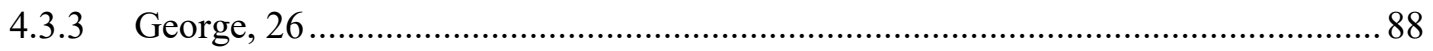

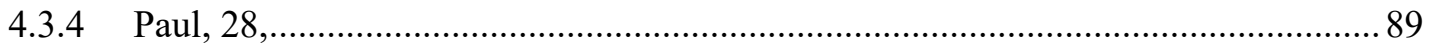

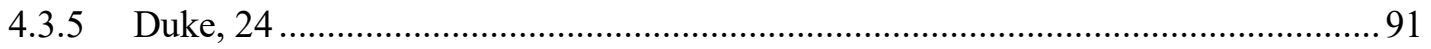

4.3.6 Themes and Motif: Location, Accessibility, Financial and Community. ................ 92

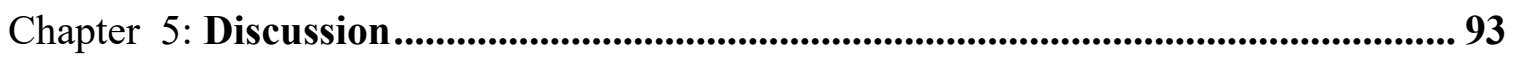

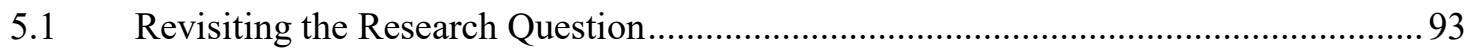

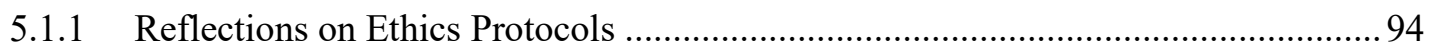

5.1.2 Legitimizing discrimination, but discrimination none the less ............................ 95

5.1.3 Attempting Factor Analysis and a lesson in failure ............................................ 96

5.1.4 Donut Charts -Affiliation, Most Concerning and Unnoticed ................................ 100

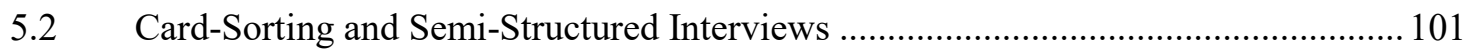

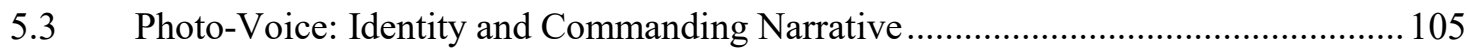

5.3.1 Location, Accessibility, Financial, Community and Self \& Agency ..................... 110

5.4 SQ1: What is the relationship between Design Activism and Social Innovation? (In the context of LGBTQ Communities living with, affected by Invisible and Episodic

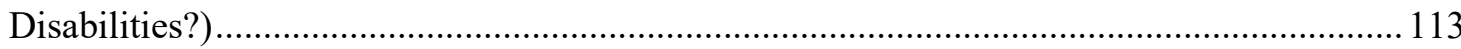

5.5 SQ2: What barriers or limit exists that result in the discouragement Design-Activism?

5.6 SQ3 How might the considerations of Critical Disability Studies and Queer Theory

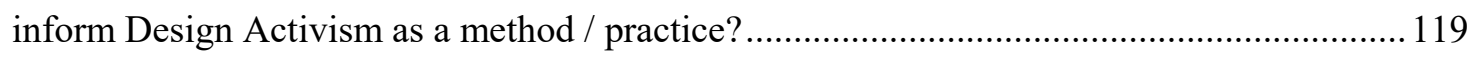

5.7 Summary: Design Activism as an Interdisciplinary Faction of Design..................... 122 
Chapter 6: Conclusion: What is Design Activism?

6.1 LGBTQ, Disability and Social Innovation and the Micro-Manifesto 125

6.2 Future Recommendations. 128

6.3 Final Self-Reflections 130

Appendix B Oral Script for Card-Sorting Interview 134

Appendix C Terms and Definitions Online Form + Consent . 137 Appendix D Participant Response Email Template 140

Appendix E Photo-Voice Online Form and Consent Form .............................................. 141

Appendix F Recruitment Email for Service Providers and Initiation of Study...... 143

Appendix G Visual Sorting Card- Activity Deck - Set Up ...................................... 144

Appendix H - Factor Analysis Source Code .......................................................... 145

Appendix I) Final Sorts of All Participants Users and Providers ........................... 145

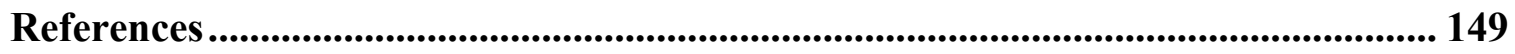




\section{List of Tables}

Table 1 Self-Reported Familiarity of Terms..................................68

Table 2 Factor Analysis Results...........................................69

Table 3 Gay Sub-Categories.............................................. 70

Table 4 SWOT Analysis of Semi-Structured Interviews............................ 76

Table 5 Low and High Priority x Service Provider and Service User .................88 


\section{List of Definitions}

The list of definitions comes the researcher's own tacit knowledge and understanding of terms. For your own benefit, readers are advised, to keep a loose interpretation of these term too. Alternatively, some definitions are provided with more clarity from reputable sources, and this will be noted within each term, when possible.

Accessibility: A multi-modal terms often associated to physical environments and ecologies, in references to one's capacity to enter and engage in a contextualized space.

Activism: Forceful campaigning targeting desirable social or political change.

Cis Gender: A person who identifies with gender assigned at birth.

Co-creation: Any act of collective creativity, shared by two or more people. (Sanders and Stappers, 2008).

Co-Design: A broad term, referring to collecting creative collaboration between designers. (Sanders and Stappers, 2008) and has specific instances in reference to cocreation.

Crip Theory/ Aesthetics: An interdisciplinary field focused on queerness and disability as site of critical examination. The word "Crip" is a nod to be an "insider" of the world of disability, appreciating and respecting all forms of disability and its cultures and aesthetics. (McRuer, 2006)

Critical Disability Studies: An interdisciplinary field that challenges traditional notions of disability as form a pity and victimization stances to critiquing social and institutional structures as enforcers of disability as a means of control. It centralizes the perspectives of people living with disabilities as experts in understanding their own disabilities and environments. (McRuer, 2006)

Decolonization: The act of returning and respecting the ownership of traditional knowledge makers. In a Canadian context, it is often associated with Indigenous and Aboriginal groups. In other social circles it is associated with departure of European centrism and colonialism as the standard of beauty, ideas and social norms.

Design Activism: A growing field of study and practice within the design communities that contribute to challenging and evolving the definition of design.

Design Thinking: A visual focused approach which aim to grapple complex challenges

Entrepreneur: An individual who engages in activity of entrepreneurship.

Entrepreneurship: The act of relocating resources to create monetary benefit from the exploitation and utilization of chosen allocated resources. 
Episodic Disability: "Long-term conditions that are characterized by periods of good health interrupted by periods of illness or disability. These periods may vary in severity, length and predictability from one person to another. Some common examples of episodic disabilities include multiple sclerosis, arthritis, diabetes, HIV/AIDS, hepatitis C, chronic fatigue syndrome, migraines, chronic pain, some forms of cancer and mental illness. Unlike permanent disabilities, episodic disabilities are periodic - the episodes of illness come and go — but because they are also unpredictable.” (EDEN Canada n.d)

Exclusion: The removal of dignity as a means of undermining, omitting and denying groups and individuals from meaningful involvement among and with their built and social environments, as well as their communities.

Heterosexism: A belief that heterosexuality is the norm and/or superior to all other forms of sexuality, whereas other sexualities may be considered abnormal, unnatural or not considered at all (Mulé et al, 2009).

HIV / AIDS: Human Immunodeficiency Virus / Acquired Immunodeficiency Deficiency Syndrome.

Inclusion: A sense of belonging and purpose that acknowledges the differences and variances of identities of people as collective whole, rather than as divisive boundaries.

Invisible Disability: A condition that cannot be detected visually, often synonymous with episodic disability, but not completely similar.

LGBTQ: Lesbian, Gay, Bi, Trans and Queer. Other variations include LGBTQ+, 2SLGBTQ, and LGBTQI. $(2 \mathrm{~S}=$ Two Spirited and I = Intersex $)$

Neoliberalism: An ideology that shifts the responsibility of the state on to its citizen, making any forms of power imbalances between any entities almost indistinguishable. Often used in context of "Privatization" and "Deregulation" of enterprises (Julier, 2013).

Ontario Disability Support Program (ODSP): A social assistance program administered by the province of Ontario's Ministry of Community, Children and Social Services. The program offers financial and employment supports.

Queer Theory: A field of study that is seeks to destabilize power through challenging notions of static identity, sexuality, and binary labels by challenging the norm.

Service Provider: An individual who operates in a professional capacity to offer care or support for service users.

Service User: An individual who access professional services from a non-profit or community based / health-based organizations. 
Social Determinates of Health (SDOH): refer to a specific group of social and economic factors within the broader determinants of health. These relate to an individual's place in society, such as income, education or employment. Experiences of discrimination, racism and historical trauma are important social determinants of health for certain groups such as Indigenous Peoples, LGBTQ and Black Canadians. (Government of Canada, 2019)

Social Enterprise: A business activity operating with the intent of creating social innovation or reduce, combat or eliminate society's most challenging issues.

Social Innovation: A practice of achieving and executing solutions that address social needs in more creativity and improves methods than the current ones. Mainly activities and services that are motivated by the goal of meeting a social need (Mulgan, 2006).

Sustainability: The enduring and lasting conditions that support survival of humans and planet without the exhaustion or over exploitation of natural resources.

that are ill-defined through a process of empathy, definition, ideation, prototyping and testing.

Trans Gender: A person who does not identify with their assign gender at birth.

UN Convention on the Rights of Persons with Disabilities (CRPD): An international treaty which shifts the view of persons with disabilities as "objects" of charity, medical treatment, and social protection towards viewing persons with disabilities as "subjects" with rights, who are capable of claiming those rights and making decisions for their lives based on their free and informed consent as well as being active members of society.

(UN.ORG, n.d). 


\section{List of Figures, Graphs and Images}

Figure 1.0 Literature Review Visual Tool Guide..................................20

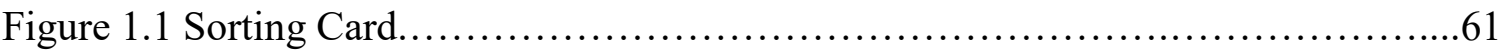

Figure 1.2 Age of participants from Terms and Definition Participants.................65

Figure 1.3 Service User Familiarity - Likert-Type Scale ................................66

Figure 1.4 Service Provider Familiarity - Likert-Type Scale ..........................67

Figure 1.5 Sree Plot Graph.....................................................

Figure 1.6 LGBTQ Community Affiliation of Participants..............................68

Figure 1.7 - Q23 from Terms and Definition Questionnaire ..........................69

Figure 1.8 - Q25 from Terms and Definition Questionnaire............................70

Figure 2.0 Final Cart-Sorting of Service Providers - High and Low Priority..............73

Figure 2.1 Final Cart-Sorting of Service Users - High and Low Priority.................. 74

Figure 2.2 Card Sorting Process Flow Chart Comparison..............................79

Figure 2.3 Service Providers' Final Thought .......................................... 81

Figure 2.4 Service Users' Final Thought........................................... 100

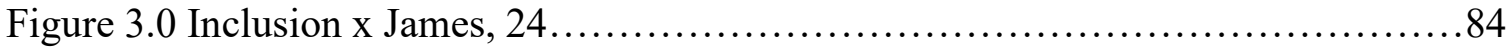

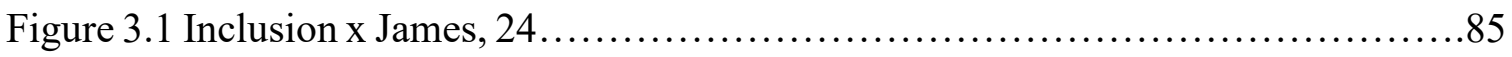

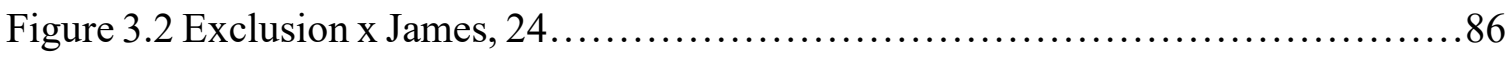

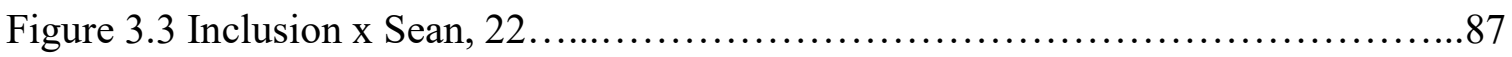

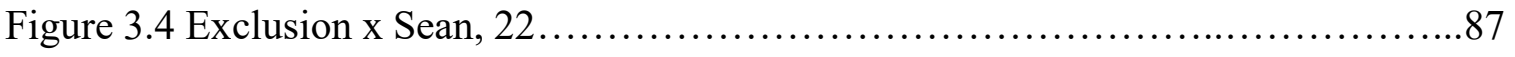

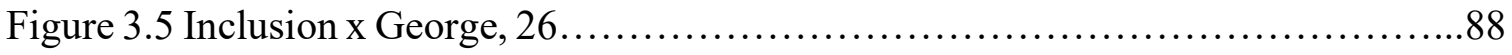

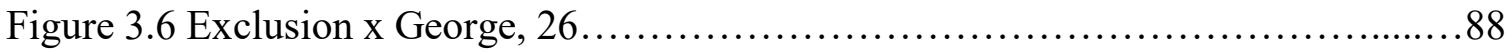

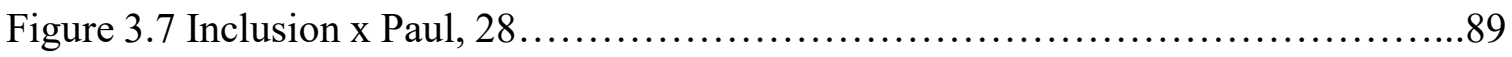

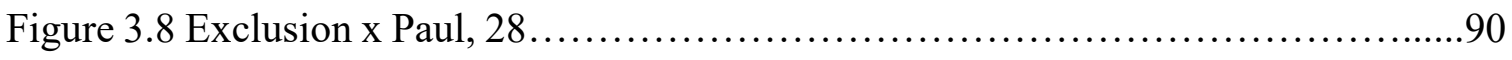

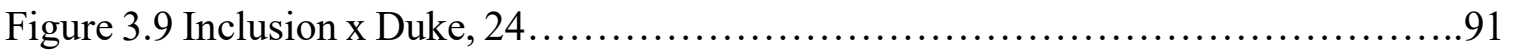

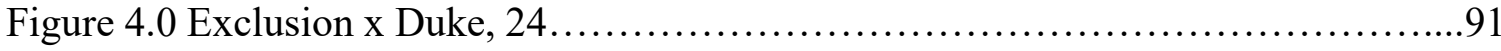

Figure 4.1 Content Map of Photo Voice........................................... 92 


\section{Chapter 1: Introduction}

\subsection{Background, Starting Point, and Original Motive}

This research explores the criteria of Design Activism in the context of LGBTQ people living with episodic conditions and invisible disabilities and its relationship to Social Innovation. The aim of this research was to expand and explore on narratives which are dismissed due to histories and stigmas associated with LGBTQ people. This research originally intended to challenge current attitudes against LGBTQ people by capturing emerging sentiments towards disability related issues, social inclusion and activism through the perspectives of practicing LGBTQ designer and LGBTQ service organizations. Considering the recent change in Ontario's provincial government, (Boisvert and Xing, 2018; Block, 2019; The Canadian Press, 2018; Income Security Advocacy Centre, 2019) the legacies, initiatives and research efforts of the previous government have been scrapped, compromising the quality of living of Ontario's Disability Support Program (ODSP) Users by modifying proposed increase in financial allowance; even changing the definition of disability; and even further disregarding LGBTQ people by completely reverting to an out of date sexual health curriculum. The researcher, as an ODSP user and LGBTQ identified individual, can assert that they are strategically advantaged to research this area from a new and Designer-ly (Cross, 2006) perspective.

Originally, this study aspired to understand how Design Activism can utilize entrepreneurship to facilitate the disruption of poverty within LGBTQ communities. Although no longer directly addressed within this study, the same tenacity and curiosity continue to drive it. . 


\subsection{Framing the Research Problem}

The goal of this section is to encapsulate the complexities and "wickedness" of the LGBTQ Communities, Disability and Designer-ly (Cross, 2006) concern such as innovation related research. Gaining further insights from existing literature reveals topics related to social services and activism. Explored are considerations of LGBTQ community cultures with an emphasis on entrepreneurship, disability, activism and queerness. Topics considering within this study include concerns such as the medical vs. social models of health and LGBTQ consumer culture.

The historical context of LGBTQ people demonstrates the saturation of literature skewed towards the medical context. LGBTQ people have been studied as health risks, complicating the understanding of how these populations maintain good health outcomes. (Colpitts \& Gahagan, 2016.) Since LGBTQ identified populations are no longer classified as individuals with mental impairments based on sexual orientation, the future of LGBTQ health and LGBTQ research, has become a vast and more daunting subject with a major potential for future research (Dukes, 2011).

In a Canadian context, a content-analysis of federal level documents, website scans and interviews reveal and render the LGBTQ people almost invisible, with a cultural understanding that there will be no further action to change the current circumstances. Its review revealed that LGBTQ people are mainly identified and mentioned within the documents of Health Canada and the Public Health Agency of Canada, mainly relating to HIV/AIDS with disregards to other health concerns. This approach fails to understand the cultural nuances of LGBTQ issues but gains deep cultural insights from the interviews. This review confirms the federal government 
heterosexists views by revealing that "LGBTQ communities are not considered a part of the social determinants of health model. When recognized, the LGBTQ populations are seen as "illness based" (Mulé \& Smith, 2014; pg. 242) rather than social, focusing on treatment rather than vulnerabilities (Mulé et al, 2009). An example is in its funding for programs primarily focused on campaigns for public awareness targeting Sexual Transmitted Infections and MSM (Men who have sex with Men) passively implying to the public that LGBTQ people are unsafe (Mulé \& Smith, 2014). De-medicalizing LGBTQ people has been advocated as critical and an important step to improving the quality of living for LGBTQ people (Eckhert, 2016).

Misconstrued for progress, the implementation of S/GBA+ (Sex and Gender Based Analysis) has been introduced at the Federal level, policy development continues to fail LGBTQ people by narrowly promoting programing targeting MSM, which contributes to the additional marginalization of the other identities of the lesbian, bi and trans communities, solely focusing on the gay demographic groups, stratifying the complexities and challenges (Mulé \& Smith, 2014). Through current cultural understanding, it is highly assumed that LGBTQ is representative of a singular community, where in fact it should be better known as LGBTQ communities (Meyer, 2016). Among LGBTQ communities, activist efforts towards gay marriage mask greater concerns around inequality. The idea of affluent LGBTQ people participating in marriage undermines other concerns around wage gaps and health disparities (Hollibaugh and Weiss, 2015). To expand further on the illusion of progress, media representation plays a role, a critique of the original hit TV series, Queer Eye for the Straight Guy demonstrates a challenge with LGBTQ identity and communities. While its production 
may be interpreted as a victory for LGBTQ representation, it continues to highlight that LGBTQ concerns are focused around the identity of gay white cis-gendered men (McRuer, 2005).

Queer theory brings a stronger understanding in participatory design and codesign, as it helps illuminate power dynamics (McWilliams 2016). By identifying these power dynamics and addressing the political agendas and roles, queer theory demonstrates its value as it is applied into the role of activism, providing "tools to mix and hybridize" (Northridge, Mcgrath, and Quan-Krueger 2007; pg.461) it is an attempt to destabilize sustained power structures on multiple levels. "Queer Theory knowns no, nor respects any boundaries or margins. Change and the invention occur at the margins, not at the center" (Northridge, Mcgrath, and Quan-Krueger 2007; pg.461) ultimately, design [activism] is about not just about good design, but challenging and changing behaviors with design (Martilla, 2011).

Studies with LGBTQ people have explored alternatives to sexual health such as working conditions, built environments and attitudes towards homophobia as needed possibilities (Willis 2010; Webster et al 2018). By introducing critical disability and queer theory as an accomplice of design, specifically participatory design methods and co-design, it can "level the playing field" by disregarding traditional roles given by institutions. Designers can contribute to social innovation beyond conceptualizing ideas. The participation of designers in non-traditional practices expands the definition of design as a social practice (Zajzon, Bohemia and Prendville, 2017). The issues around pro-bono work does invite questionable behaviors and attitudes, considering design is a profession, however, pro-bono design projects allow community groups and 
organizations to benefit from design-thinking. A key important principle involves giving the community ownership, presence and space. The participation and contribution of community-based projects as a designer is best understood as a mutual rewarding relationship. Through pro-bono design work, the opportunity for new and alternative learning is achievable. This practice is a method of acquiring new skills and application of entrepreneurship and community development (Shea 2012). The solutions to social innovations do not always need to be new or novel, but it must bring ideas into a new territory (Nicholls and Murduch, 2012). A benefit from social innovation is its focus on capacity building and leadership (Alvord, Brown and Letts, 2004). From a capacities approach, it helps understand the relationship between marginalization and social innovation. Areas in Europe have advocated for using social innovation for tackling marginalization. To truly understand social innovation, it is best realized by acknowledging its shift from economic development to human development (von Jacobi, Edmiston, \& Ziegler 2017).

Additional thematic comparison between LGBTQ literature and Social Innovation literature reveal a similarity which champion the idea of there never being singular "center" points of view. Marginalization is not meant to be interpreted for its traditional negative connotations. Rather marginalization is a vantage point offering a fresh perspective. Emerging from this review reveals that queerness has much to do with social innovation; there are no static boundaries. Entrepreneurship, Activism and LGBTQ people share commonalities as responsive agents to societal issues, however, share different motivation; concepts such as survival and territory resonate in all areas of literature yet express their concerns uniquely within varying context. 
By exploring the blurring points, and where the values align between LGBTQ issues and Social Innovation, it may provide a deeper understanding of Design Activism. Within LGBTQ and Social innovation literature, studies in critical disability and design are tempted to converse. While design begins to adopt concerns located in disability studies, it can be assumed that this relationship is one directional. So, how might disability adopt from design, and vice versa? And is that even necessary? Considering the heavy reliance of visual cues within design, how might design fail or prevent itself from acknowledging other ways of knowing. Impairment and limitations, like, disability and inability, are not the same, they just offer a different path to discovery. Current attitudes towards disability in design, specifically universal design, fail to understand its true potential, thus these failures are opportunities as queer theorists and critics have mentioned. Following Halberstam (2011) and Muñoz (2009), both attribute failure as an underestimated adventure few choose to explore. There is a saturated fascination and skewed definition of success, contributing to a repeated cycle of ignorance and complacency. Rather than accepting disability as a deficiency, accepting disability as an alternative way of knowledge generation is an indicator of respect; appreciating disability as a movement towards social justice, values the power of difference.

Queer Theory and Critical Disability Studies, share a lot in common; exploring and shattering misconception of what it means to be "broken." Unbecoming, and unknowing, provide explosive imaginary possibilities. It is often the logics of neoliberalism and capitalisms that mask and deny access to alternative metrics of understanding and knowing. Queer Theory and Critical Disability studies, champion and support dissent, as a needed condition of design activism, and as a necessity to facilitating 
new ways of knowing.

Exploring the queering of disability and design warrants further investigations and would be a meaningful contribution for "designer-ly" (Cross, 2006) ways of knowing. Especially considering Questioning whether this relationship between queer disability and design offer mutual rewards, heighten the possibilities of inquiry as it explores the "In-Between" living and "moving back and forth between embodied states of identities" (Vick, 2012; pg. 46). Queerness, Entrepreneurship, and Activism have overlapping issues regarding its conceptualization of risk, riskiness, and collective organizing. The intellectual territory of design and queer disability are complex systems, and promises unknowing, failure, and dissent as forms of knowledge yet to be imagined or accessed.

\subsection{Study Objectives}

This study's objectives are to (1) understanding Design Activism, when considering LGBTQ community organizations by borrowing from Queer and Crip Theory and Critical Disability Studies, through literature, aesthetically considerations and its relations to social innovation, (2) expanding on design research methods as a form of design activism itself by introducing card-sorting into various LGBTQ social service settings, a SWOT analysis and photo voice/journaling and factor analysis, (3) critically examining the impacts and criteria of Design Activism in practice with LGBTQ communities and Disability in deliberation by co-creating and designing artifacts with LGBTQ community members as partners. 


\subsection{Research Question}

Q1: What is Design Activism in the context of LGBTQ communities, living with invisible disabilities and episodic conditions?

SQ1: What is the relationship between Design Activism and Social Innovation? (In the context of LGBTQ Communities living with, affected by Invisible and Episodic Disabilities?)

SQ2: What barriers or limit exists that result in the discouragement Design-Activism?

SQ3: How might the considerations of Critical Disability Studies and Queer Theory inform Design Activism as a method / practice?

\section{Chapter 2: Literature Review}

Below is visual tool intended as an abstract, marking connections between literature. Its function is not intended to be interpreted as an absolute articulation, but as loose guide.

\section{Literature Review Visualization}
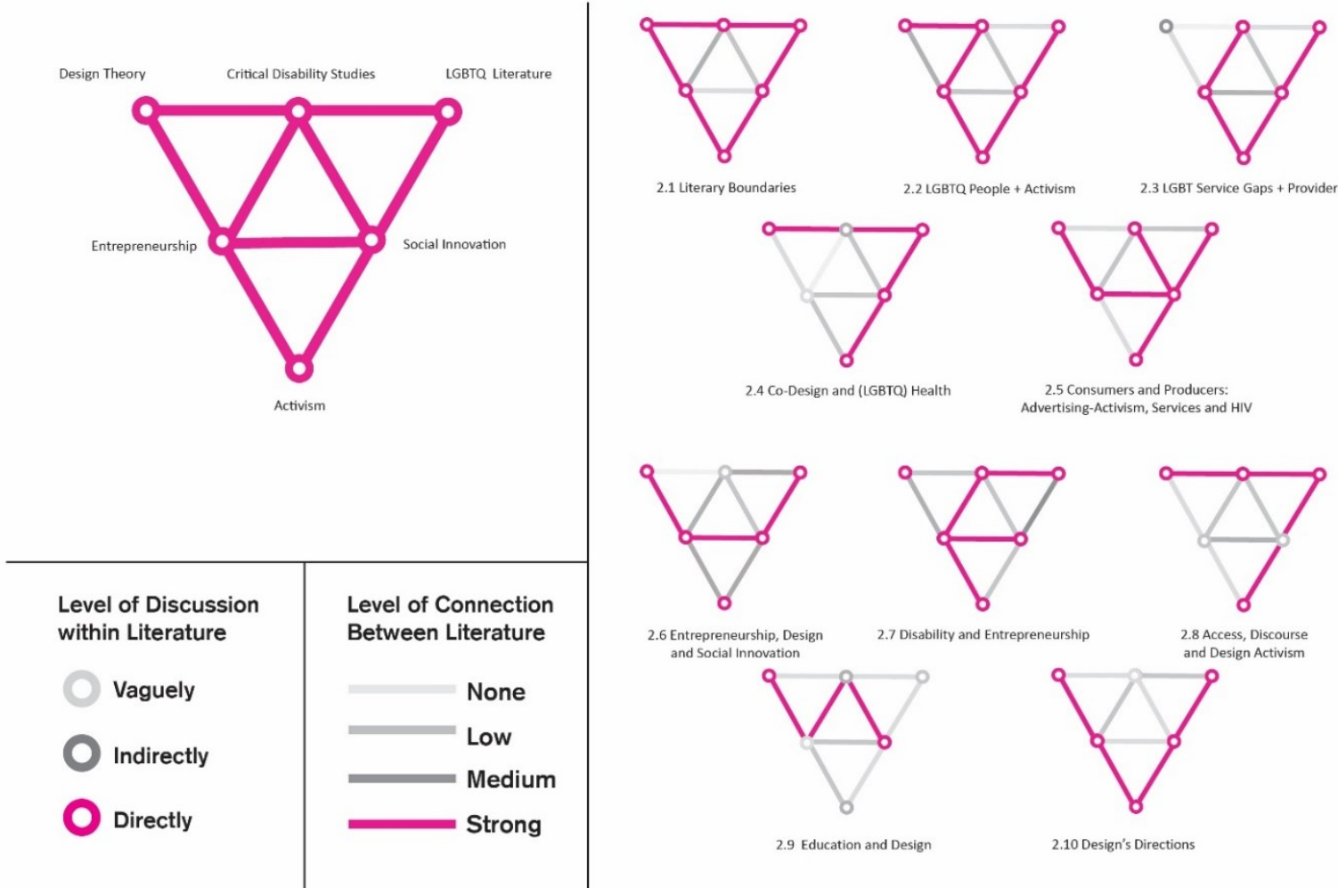

2.1 Literary Boundaries

2.2 LGBTQ People + Activism

2.3 LGBT Service Gaps + Providers

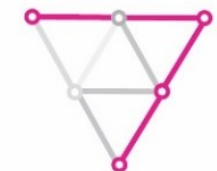

2.4 Co-Design and (LGBTO) Health

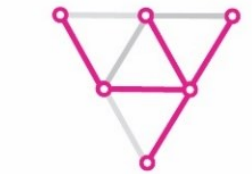

2.5 Consumers a d producers: Advertising-Activism, Services and HIV
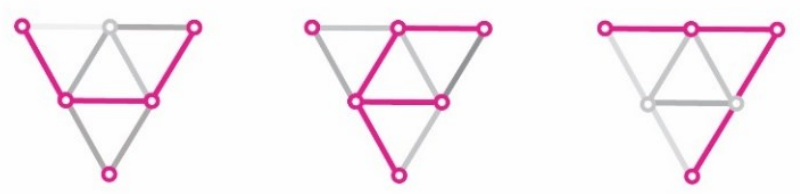

2.6 Entrepreneurship, Design

2.8 Access, Discourse and Social Innovation
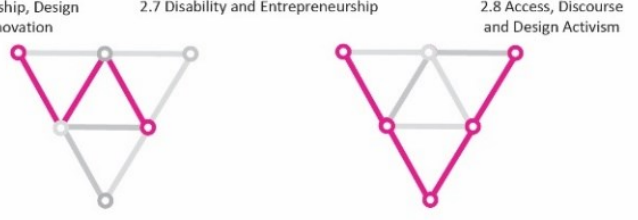

2.9 Education and Desigr

2.10 Design's Directions

Figure 1.0 - Literature Review Visualization 


\subsection{Literary Boundaries}

This literature review bridges literature from a range of fields, from social innovation and entrepreneurship to LGBTQ+ Health, Disability, HIV and Public Policy and Design Theory. Although not exhaustive, the intent of this literature review is to capture the complexity of LGBTQ+ culture and its conflicts as it is articulated in existing research, to better understand how it may benefit or inform Design Activism. This literature review is divided into three major segments. First, taking a sharp departure from LGBTQ activism to social policy, then turning towards social service considerations. This is to contextualize and to understand the starting point of LGBTQ+ people, its history of activism as it relates to community-based research (codesign/participatory based research) as a strategy for engaging LGBTQ+ populations. Secondly, exploring social innovation, design and entrepreneurship as alternative pathways of addressing marginalization. This section explores how entrepreneurship has forcibly or indirectly shaped the world and LGBTQ+ communities and disability under the context of capitalism. Last, exploring the context of Design theory, praxis and future directions. This review concludes the importance and interconnectedness of LGBTQ+ identity to disability, design and social innovation as it relates to the futures of LGBTQ+ people living with invisible and episodic conditions and the practice of Design Activism.

\subsection{LGBTQ People and Activism}

LGBTQ+ activism history documented in literature presents a range of emotional intensity (Gould, 2009). Activism within LGBTQ communities is a direct response to persecution and attacks from the state and religious institutions. While gains in developed countries are worthy of celebration, much of other parts of the world remain behind. 
Advances and momentum were gained during the 60 s and 70 s with homosexuality no longer classified as a mental illness. Eventually, LGBTQ activism would be disrupted and invigorated by the discovery of HIV/AIDS (Gould 2009; Elbaz,1995). This disruption was transformative to activism, revealing hidden power dynamics between governments, corporations and LGBTQ people (Elbaz,1995). Since the 80s and 90s, new science and knowledge has been produced. However, the challenge that remains involves access to medications globally (UNAIDS.org, 2018) and LGBTQ pursuit of equality. This is completely underscored by LGBTQ people constantly encountering health disparities compared to their heterosexual peers (Meyers and Northridge, 2003; Northridge, McGrath and Quan-Krueger 2007; Mulé and Smith, 2014; Kelleher, 2009).

LGBTQ as an umbrella terms assumes this community is unified as a singular community identity.. Studies on LGBTQ people, often in medical and demography studies, suggest that LGBTQ people can be better represented and understood as a range of communities with sets of subs set identities and subcultures (Meyer, 2016). Argued by Warner (2004):

Queer research should stop adding letters to LGBT research and should instead form a body of knowledge about how these categories come to be, and are lived, on a daily basis. To queer methods and research is understanding that knowledge can be produced within the margins; seeking social knowledge in a biological framework is a journey to failure (pg. 335.)

Illustrated within this quote is the expansive and growing communities of LGBTQ people, complicated by its need to be studied, as these groups are mainly studied from a medical perspective (Mulé and Smith, 2014). The identities of Lesbian, Gay, Bi, Trans and Queer people often form the commonly known form acronym as LGBTQ. Often associated with "+" sign and is sometimes a perceived invisible plus sign. The expansion 
of this acronym is a reminder of the ever-evolving understanding of identities within these sub-set of communities and should never be assumed as singular identity (Meyer, 2016). Fredriksen-Goldsen's (2016) article on research and policy highlights the importance of generational changes and that language becomes an indicator and reflection of societal values. In some cases, other forms of the acronym are expressed as, LGBT2SQ, 2S, meaning Two-Spirted, addressing the gender and sexual dynamics of aboriginal and indigenous groups. In some cases, there is an "I" at the end representing Intersex people and an " $\mathrm{A}$ " for asexual.

Issues around homophobia and transphobia have antagonized the LGBTQ community for a notable period of the last few decades. Using sexual orientation and gender expression as a marker of minority or marginal status is evident that people, inherently aspire to create multi-tier values systems of people. This is best expressed in a critique by Roberts and Marx (2018) on protectionist policies and LGBTQ advocacy research. Roberts and Marx (2018) challenge protectionist policies by asking: "How can queer people survive and thrive when the law views them as second class - a lower citizen whose behaviors are so aberrant and repulsive that they must be regulated at every turn?" (pg. 2) The rhetoric and logics of protectionist social policies that aim to support LGBTQ communities contradict their own purpose. Roberts and Marx (2018) expand further, borrowing from Foucault's $(1988,1978,2003)$ theories of disciplinary power and biopower, and apply these concepts vis- à-vis LGBTQ youth who are bullied in school, by highlighting that protectionist policies are investments of power. More specifically, three aspects of sustained investments of power are done by: universalizing the individual, prioritizing individuals over structural change and, finally, addressing 
inequities through punishment and surveillance. These protectionists policies encourage silence, inadvertently, reproducing exclusion for queer bodies and anyone outside of the majority (Roberts and Marx, 2018). Other examples include, GLSEN's (2018) study on promotion of homosexuality within public schools, and its relationship to state regulation. Its review highlights that schools that are located within states that support health promotions of LGBTQ people encounter more frequent political hostility than schools in states that do not support LGBTQ health.

Despite failures to cease, forms of discrimination against LGBTQ people, form of service and policy innovation exist from within LGBTQ communities (FredriksenGoldsen, 2016). Activism continues to play a critical role in changing health and social policy, more importantly, it has challenged the definition of access regarding medication and even same-sex marriage. (Elbaz,1995; Fredriksen-Goldsen, 2016).

\subsection{LGBTQ+ Service Gaps and Service Providers}

The rise of LGBTQ health services has a direct connection to the rejection, mobilization and protest of homophobia, particularly due to homosexuality becoming declassified as mental illness. The advancement of LGBTQ liberties means equal consideration for access of services. Although there has been an increase for LGBTQ health services, it continues to be neglected among general population health concerns, often as an afterthought (Martos, Wilson, and Meyer 2017). Limited considerations can create and expand service gaps due to cultural incompetency. Furthermore, a service provider's heterosexual identity itself has been documented as a barrier for practicing empathy when caring for LGBTQ people, especially for youth. A clinician's social location and heterosexual identity can foster cultural incompetency, in both professional 
and personal interactions. These social barriers can contribute to patients feeling guilty and disempowered. For care provers, feeling anxious and inadequately skilled can lead to poor assessment and treatment (Knight et al, 2014). More precisely, Knight et al have argued that:

Bringing the 'social' into the clinic represents an important opportunity to address influences that render individuals and populations vulnerable to inequitable social and health outcomes; as a result, integrating practices that address social issues (e.g. heterosexism) within clinical practice have the opportunity to decrease health inequity not only among LGBTQ youth but also among older generations of LGBTQ (pg. 668).

In many medical curriculums, doctor training and competencies remain unaudited for inclusion of LGBTQ content curriculum, contributing to further service delivery gaps (DeVita, Bishop, \& Plankey, 2018). Additional consideration around gender and racial discrimination and the experience of racialized workers are also a site of conflict and barrier to access (Greene et al, 2015; Giwa \& Greensmith, 2012; Logie \& Rwigema, 2014; Kattri, 2017). Championing for safe and inclusive spaces has shown to have major benefits for LGBTQ people, it has demonstrated impact through improved access to services as suggested by Martos, Wilson, and Meyer (2017) especially for youth, particularly in the area of suicide contemplation as noted in Saewyc et al's (2014) suicided related study. When shifting the focus on older LGBTQ people, it is important to know that older LGBTQ people have experienced higher concentrations of repressive politics, meaning, generational differences may present themselves as a barrier to inclusion, and that the struggles of today's LGBTQ communities may not be the same as the previous generations. Understanding resiliency in previous generations of LGBTQ communities is vital to understanding how resilience is conceptualized historically and 
invites questions regarding its relevancy to younger generation of LGBTQ people, many whom have not experienced the same forms of political oppression. (Brotman et al, 2015).

When exploring concerns with LGBTQ population research, the most notable method that emerges is community-based participatory-research (Northridge, Mcgrath, and Quan-Krueger 2007; Institute of Medicine, 2011; RHO 2012,). Community-based methods share a bottom-up approached rooted in the spirit of advocacy and activism, contributing diverse perspectives, thus beneficial to for Public Health agencies and everyone. These participatory methods have been useful to improving health services and research among LGBTQ communities (Northridge, Mcgrath, and Quan-Krueger 2007; Logie \& Candice Lys; 2015; Winterbauer, et al. 2016).

\subsection{Co-Design and (LGBTQ) Health}

Participatory methods have a connection to Co-design and is not all that new of a practice (Sanders \& Stappers, 2008). Variations of its practice can be found as early as the 60s from literature exploring Scandinavian democratic design approaches, with a focus on social wellbeing (Guffey, 2015; Lie, 2016). More specifically, Sanders \& Statppers (2008) state "Co-design refers, for some people, to the collective creativity of collaborating designers. We use co-design in a broader sense to refer to the creativity of designers and people not trained in design working together in the design development process" (pg.6). Yin et al, (2017) suggest that health goes beyond the individual level, public health is a social concern, and desired outcomes can be achieved through extension of co-design and co-creation. Design plays a critical role in the future of healthcare due to its capacities to define parameters and identify limitations, and it will be 
even further impactful through co-design and co-creation (Chamberlain \& Partridge, 2017). While innovations may be pursued through design methods, designers must be aware that they too play a critical role in facilitating the process. This can be achieved when a designer's practice rejects its monopoly on design, as suggested by Manzini (2007). Emancipating from dominating practices will lead to diverse network which resonates with what Sanders \& Statppers (2008) describe as the new landscape of design, meaning, by releasing power and traditional roles of design, designers are changing as designers and shifting into "hybrid roles of design researchers and researcher designers." (pg.15.)

\subsection{Consumers and Producers: Advertising-Activism, Services and HIV}

When exploring the overlapping of, LGBTQ inclusion and Design, much can be learned from the domain of advertising (McNamara and Descubes, 2016; Branchik, 2002; Raley and Lucas, 2006; Tsai, 2004). Advertising becomes critical in reflecting societal values, inviting opportunities to comment and create new ways of knowing. Through exploring video content and prime time TV, with such shows as Will \& Grace and Friends, LGBTQ representation continues to build momentum by introducing LGBTQ characters in major film and television productions (Raley and Lucas, 2006). As a result, much of this is becoming more reflected in advertising. McNamara and Descubes' (2016) study on Targeted Marketing to the LGBT communities contends that the rise of LGBTQ consumerism relates to the progress and advancement of LGBTQ civil rights in the US. Specifically, notable changes occurred during the 1970s as advertisers noticed the "economic value of the gay community" (pg. 2). Prior to the 70s, a majority of LGBTQ advertising came from LGBTQ owned businesses (Branchik, 2002). Major areas of 
LGBTQ advertising came from product and service categories in hospitality for sectors such as bars, restaurants, brothels and bathhouses. Outside of this category, clothing, was another dominant product category, targeting LGBTQ people as clothing and fashion enabled hidden cues to signal and communicate one's LGBTQ identities without repercussions. LGBTQ people have been inadvertently influencing the market of products and services, thus revealing a behavioral pattern of study interdependent to various facets of society. (Branchik, 2002). In 2013, a study by Witeck Communications (2013), estimates the purchasing power, money deemed as disposable income, of LGBTQ consumers in America was near the total of $\$ 830$ billion.

Report by LGBTQ Capital, shared by INTO (2018), a millennial-focused digital platform, suggest that most companies continue to miss this opportunity to reach this audience. The US LGBTQ domestic spending is now valued at $\$ 965$ million (USD). On a global market, LGBTQ communities command a total of $\$ 5$ trillion in spending power. This target audience continues to grow and become an area of major interest; LGBTQ people as consumers are lucrative, not only influencing market demand, but also forecasting future consumer trends.

When connecting back into the context of LGBTQ youth, digital spaces become arena and site for engaged consumer discourse, LGBTQ youth are discovering themselves and their politics in digital spaces (Gray, 2009; Middaugh, Bowyer, \& Kahne, 2017) and in some cases, through music playlists and pornography (Harper et al, 2016; Dhaenens \& Burgess, 2018; Aronoff \& Gilboa, 2015). Digital spaces become an important form of access because they allow communities to grow, connect and discover one another. This can include such digital platforms such as Reddit Forums, Spotify 
Playlists, and YouTube Channels. Although consumer centric, participation on these platforms generate and contribute the evolving definition co-creation and co-design (Sanders and Stappers, 2008). These individuals play a passive role, since they do not own the platform, and through its participatory involvement, greater potential can be found in "interactions and the use language" (Miller, 2017; pg. 522) as it can contribute to forming identity, because in online spaces, participants exercise choice, decisions making and social interactions, whether conscience of it or not. Further, LGBTQ youth in digital space are seeking information that is not so readily available to them, such as sexual health and romantic interactions. Through these digital spaces, LGBTQ youth are seeking validation, affirmation and "managing identities" (Miller, 2017; pg. 516).

While diversity of such identities is important, a major challenge within LGBTQ research is that much of it is focused on gay, cis-gendered, white men, or the denial of racism within its communities (Balsam et al, 2011). Considering that LGBTQ people are continuously challenged by health disparities (DeVita, Bishop, \& Plankey, 2018), the development and awareness of these issues need to be heard beyond medical institutions with public awareness campaigns. While advertising focused on public awareness regarding social inclusion of LGBTQ people is crucial, understanding a culture's readiness of change is just as important (Gheorghe, 2016). Another area of concern involving the creation of LGBTQ awareness campaigns is its understanding of evaluation. Consumer research suggests measuring and encouraging behavior change or attitude change as a means of confronting underlying beliefs and cultural regimes. (Gheorghe, 2016).

In the process of developing advertising content for LGBTQ audiences, the 
intentions of the advertiser and who they portray play a critical role (Branchik, 2007). Much more is still left to be discovered in LGBTQ advertising and marketing, this is due to its field of research being in its infancy (Ginder and Byun, 2015; McNamara and Descubes 2016). Such an infancy is an indicator that much more can be done to advance LGBTQ research and community growth. Advertising and communications design play a larger role in society due to its access in public spaces. It can inform both practitioners and academics about the culture and the changes within the market. Advertisements themselves serve as relics and indicators of the era's current social and political tensions (Branchik, 2007).

For those considering creating LGBTQ campaigns, it is much advised to consider the negative consequences of producing such content; negative meaning, the anticipation of hate speech and acts of protest. Another area of concern within the area of LGBTQ advertising and marketing is backlash from heterosexual communities, more specifically, heterosexual consumers (Tsai, 2004). Companies that are not genuinely LGBTQ friendly would be advised not to present themselves as such, for LGBTQ communities are historically known for their capacity to self-organize and discover hidden agendas (Gheorghe, 2016).

Calling on a to a Queer and Crip perspective, Clare (2015) states: "Queer and cripple are cousins: words to shock, words to infuse with pride and self-love words to resist internalized hatred, words to help forge a politic.” (pg. 84). Then, it would make possible to imagine the strange and direction connection between advertising to activism (Heller, 2013; Lasn, 2006), as conceptually similar. Advertising and activism are more like gay twins, alike, but different, obnoxiously in your face; perhaps more like Siamese 
twins, and inescapable of each other; mirroring a politic vulnerable to replication, yet highly effective in their similarities and notably capacity to garner attention for its glaring oddity and gawk worthy invitation. Its similarities are almost freak-like, leaving it vulnerable for critique, yet yielding authority in unknown ways. It is the embracing of such oddity and potential contradiction that co-create such tension needed for critically engagements.

Historically, LGBTQ groups, specifically gay organizations such as ACT UP, a grass-roots movement of the 80s AIDS crisis, resonate with Clare's (2015) ideas of queer and cripple in action, mainly forging a politic, only to be articulated by repurposing many techniques from advertising. This was achieved by Gran Fury, a division of ACT UP, its propaganda arm, focused on creating political messaging. Even the name of Gran Fury was taken and given new meaning; its origins are of the Plymouth car used by New York Police cars during the 80s, which has even further implication with LGBTQ histories of violence (Mallory, Hasenbush, and Sears, 2015; Heller, 2013). Heller (2013) in an interview with Loring McAlpin, an original member of Gran Fury, retells "We thought the name of the squad car described our anger and urgency, with humour, a slightly camp sensibility, and a nod to the ordinary - a mid-range Plymouth" (Location, 1075 of 1194). Gran Fury's efforts are known for gaining media attention and applying political pressure for social reform and medical access (Elbaz,1995). McAlpin, shares further, regarding the acceptance of their activities and initiatives, by stating: "In general, we tried to remain aware of what was permitted in public spaces. If our passage was too radical, we risked access as well as a broader public reception" (Heller, 2013; location, 1104 of 1194). This sentiment demonstrates the negotiation of publics spaces, as public spaces became site 
and platform for protest and site power negotiations. Additionally, in relation to

advertising and language, McAplin shares:

We simply used the tools that were available to us, and of course the language of advertising and appropriation were two of the first places we looked, even as we sought to insert unexpected messages in those vocabularies... We wanted our activist voice to fill that void...therefore, we tried to insert our message seamlessly in those spaces that were normally occupied by authority, and we used whatever we could to grab attention. It didn't matter to us if that was a borrowed strategy or not

(Heller, 2013; Location, 1080 of 1194).

HIV's notable and unique affiliation with the LGBTQ communities are noted through its history of activism, part of which was fueled by the anger from pharmaceutical profiting of HIV medications (Elbaz,1995). LGBTQ people, in a western context, continue to be at higher risks for HIV acquisition (Northridge, Mcgrath, and Quan-Krueger 2007; Institute of Medicine, 2011). Historically, early medication had debilitating effects, due to toxicity, thus categorizing HIV as a disability. Now, medical advances refer HIV as a chronic medical condition. In Canada, HIV is considered a permanent medical condition that makes one living with HIV eligible to apply for longterm disability benefits (CATIE.ca, n.d). In response to the medicalization of HIV and disability, an intersectionality of HIV/AIDS and disability has emerged as an important global policy development (Health Canada, 2009). By framing HIV and disability as disease oriented, it fails to underscore financial, emotional and social related challenges associated with living with an invisible and episodic conditions (Porch and Yates, 2018). Policy development introduced by Health Canada (2009) was a challenge to the medical understanding of disability and HIV, a result of the UN Convention on the Rights of Persons with Disabilities (CRPD); a policy instrument aimed to be an 
"advocacy tool to counteract negative perceptions of people living with disabilities" (pg. 12.) The instrument in effect, shifts disability from a medical understanding to a rightbased approach, describing "disability as an outcome of the interactions between the challenged person and the obstacles (social, environmental, institutional, legal) that limit full and activity participation in society" (pg. 12.) Further, the Ontario Human Right Commission (1996; 2008) highlights "AIDS (HIV) is much more than just a medical/scientific phenomenon. It challenges our fundamental values such as a commitment to a compassionate society, to justice and to the elimination of all aspects of discrimination that undermines these values" (pg. 4).

In the titled book, Seeing Red, a collection of engaged commenters on HIV policy, share multiple viewpoints on the intricacies, limits and obstacles placed on the systems of HIV care. Through policy developments, collectively, they all argue that the systems created fail to express true potential; the systems in place remains an artifact of our lost humanity and lack of justice. Seeing Red offers insight into a hopeful, yet critical horizon mainly due to HIV care and services shrouded in secrecy, through means of client confidentiality. This sector remains largely elusive due to confidentiality policies, that are assumed to protect the identities of its service users, and top-down organizational structures. These organizational dynamics politically charged, thus limiting any democratic decision-making, as its logic are outsourced into a hierarchal process, thus silencing any dispute regarding any power imbalances. These processes are then used against LGBTQ communities, people of colour, incarcerated people and indigenous and aboriginal communities. Unimaginable labour burden, which comes through the acts of remaining silent or complacency, impacts minorities and marginalized people by further 
due to their inability to react due to punitive measures, such as the denial of access of services. As a result, much of the knowledge generated within the HIV and Gay Health sector never reach beyond the professional boundaries (Hindmarch, Orsini, Gagnon, 2018). Other examples that complicate HIV and LGBTQ communities are advances of science, and the laws' capacity to keep up with science. This is evident in Canada's leading record in criminalization of HIV people for non-disclosure (Canadian HIV/AIDS Legal Network,2019.) This only exacerbated considering the federal government views LGBTQ and MSM as "illness based" (Mulé \& Smith, 2014;pg 242.) Another example which further strides the complexity of HIV and society at large are its patent and research mechanism. These systems been described as a "ticking time bomb" as argued Hoen et al (2011) meaning, the costs of medications and advances in medications will continue to rise. Treatment becomes further inaccessible to the poor and those who may be financial impaired by the cost of medication. Research systems used to assign rewards towards innovative research and medications are then hoarded by patent laws, again, further entrenching medications inaccessibility (Hoen et al, 2011). Like the arguments made in Seeing Red (Hindmarch, Orsini, Gagnon, 2018), Hoen et al, (2011) calls upon the need political activism's capacity to instigate social pressure on governments for needed change. This comes more pressing, particularly in Canadian context, as a report in 2017 revealed a total of 2,402 new HIV cases, an increase of 3\% compared with 2016 and an increase of $17.1 \%$ since 2014. (Haddad et al., 2017)

\subsubsection{Queer Business}

When exploring LGBTQ communities and economic impacts and benefits, entrepreneurship becomes a critical entry point. Often it begs the question about what an 
entrepreneur's role might be towards the greater LGBTQ communities, what obligations exist and how might entrepreneurship grant or prevent access. Irving (2009) argues, in the context of Trans identities, that "neoliberal governance takes place in large part through equating morals with economic productivity.” (pg. 385.) The capacity to work is an indicator of value and legitimacy, and that neoliberalism, as ideology, see a shift in human's citizenship to a consumer identity. Economic discourse contributes to the erasure of trans people, thus displacing and disadvantaging access to rights. In response, the means of survival, for trans identities and by extension, queer individuals, are to assimilate and sustain "insidious operations of power" (pg. 395) that perpetuate marginalization by participating in "hegemonic bargaining" (pg.375) with those with greater access to social capital and privileges.

An example of "hegemonic bargaining" demonstrated in LGBTQ communities are Pride Festivals. Originally intended as a riot and protest, Pride Festivals have evolved over the decades from a riot and protest to corporate sponsorships, as form of acquisition, and growth through means of negotiating funding and monetary capital. Such occurrences are aligned with what Julier (2013) argues about [design] activism not immune to neoliberalism, and Irving's "hegemonic bargaining" (pg.375.) A report on Pride Festivals indicate that these festivals have major economic benefits. One report commissioned by the province of Ontario, Canada's Ministry of Tourism, by PFK Consulting (2009) indicated significant economic contributions to tourism and culture, as well as civic pride and inclusion, elusive and buzz worthy ideas that can be reinvested to gain further economic gain. In effect, Pride Festivals are victims of funding dependency from corporate sponsorship. 


\subsection{Entrepreneurship, Design and Social Innovation.}

There is a striking similarity between design and entrepreneurship, primarily with the dimension to create new ideas and innovative solutions. Efforts in collaboration among diverse teams and product development are notably different between designers and entrepreneurs, as a result, it allows designers to be well situated for entrepreneurship as suggested by Nielsen, Wikström, \& Tollestrup (2013). Strangely, Nielsen, Wikström, \& Tollestrup's (2013) argument conflicts within their own study, as they imply that designers "may lack competitive aggressiveness." Contrary to this claim, design is often employed by entrepreneurship and business to gain competitive advantages; this is done by creating new tools and products; innovation for competitive gain is better understood through changing meaning. It is those who interpret the world differently which allows for further innovation (Verganti, 2009). As designers are situated and trained to envision

products and services differently, they command the language for both design and business. This is because design and business share a focus on problem definition and discovery as noted by Matthews (2009). Effectively, there will be personal and profitable gains for the generating of an ideas and adoption into market (Anderson, 2014). As a result, publication journals in the field of design, trade and academics have increasingly gained awareness of its monetary and social value; trade organizations, industry conferences and seminars are rapidly flooding the market (Anderson, 2014).

\subsubsection{Design and its relationship to Social Innovation}

Within the field of Design, a major shift needs to occur, specifically imagining beyond the singularity of issues to adopting a holistic approach (Cooper, 2005). Additionally, Rezai \& Khazaei (2017) argue for the importance to shift design from 
serving market-based form of production and consumption. Specially, Design researchers and practitioners' capacities to establish new relationships through method is important because "design is not merely an outcome of environments but also a source of production of environments" (pg. 7.) However, the argument for a holistic shift presents many barriers to adoption, specifically around replicability and intent. As enterprises and replicable models emerge from interactions from designers, these initiatives require independence from designers. As part of the creation of initiatives, all concerned stakeholders and designers need further consideration of an exit strategy; considering how a project journey ends are as equally important as its inception (Fassi, Meroni, \& Simeone, 2013).

Taking a holistic approach needs to involve understanding the process of decision making and its relation to applicable evidence (Cooper, 2005). Globally, the acceptance of design as a path for social innovation becomes questionable, for its limited replicability, resources and inconsistent forms of success metrics, which itself becomes a barrier for adoption (Shaw and Bruin, 2013; Groots \& Dankbaar, 2014; Austin, Stevenson, \& Wei-Skillern, 2006). Further complicating the debate around the path to social innovation are its focus on people vs. planet (von Jacobi, Nicholls, ChiappereMartinetti,2017). Groots \& Dankbaar's (2014) suggest that social innovation is not solely produced by social entrepreneurship; entrepreneurship can equally contribute to socially innovative outcomes. Comparatively, social enterprises, as suggested by Hechavarría \& Welter (2015) tend to be more socially innovative than "normal" enterprises. 
Defined by Mulgan, (2006) Social Innovation is "innovative activities and services that are motivated by the goal of meeting a social need" (pg. 146) thus, enterprises that focus on social need become social enterprises, but mainly in start-up context (Hechavarría \& Welter, 2015). More so, its focus and criteria are contributed to the ingenuity of people working together, particularly in times of uncertainty and economic instability (Tiwari, 2017). Criticism on the definition cite Social Innovation to be a conflated "buzzer" word, extending the purpose of communication strategies with further context and scrutiny on corporate entities; distinguishing social innovation from regular innovation is in its motivations for improvement of quality of living rather than financial profit motives. (Pol \& Ville, 2009; Cooper, 2005) Other critics of social innovation acknowledge the high levels of unnoticed labor. Social innovations are easily dismissed and devalued because of the logics of capitalism, (Mongelli \& Rullani, 2017) particularly because capitalism operates within a "Winner-Take-All" mentality, further marginalizing and perpetuating inequalities. While studies argue in favor of Social Innovation of its capacity for positive societal change (Tiwari, 2017; Nandan, London,\& Bent-Goodley, 2015), and its favorable outcome; social innovation truly occurs at the blurring of sector boundaries, thus challenging the merit of capitalism (Shaw and Bruin, 2013).

\subsubsection{Marginalization and Social Innovation}

Social innovations emerge out of failures from both private and public sectors (Austin, Stevenson, \& Wei-Skillern, 2006) and has been studied and argued for its capacity to challenge marginalization (von Jacobi, Nicholls, Chiappere-Martinetti,2017; Phillips et al, 2015; Mongelli \& Rullani, 2017). The presence of a social innovator is 
necessary as suggested by Ziegler et al (2017) and in most cases, they are often from marginalized communities themselves. Their identity and community affiliation become a distinct advantage for launching an innovative and responsive project.

\subsubsection{Design and Enterprise}

Social innovation and social enterprise have been considered a responsive alternative to social problems (Austin, Stevenson, \& Wei-Skillern 2006; Selloni \& Corubolo, 2017). Existing literature in the field of design argues that social innovation is a by-product of design-thinking (Verganti, 2009; Çeviker-Çınar et al, 2017; Martin, 2009; Brown, 2009). Design-thinking can be applied to support social innovations; however, it does come with limited formulas for consideration. A specific benefit and strength are the opportunities for new perspective on systemic thinking, which can be usually supported through visualization of complex concepts. A drawback includes unknown variable costs and limited understanding of measurable metric in a traditional economic sense. As a result, there is often a fear of adoption or a skills gap for implementation (Selloni \& Corubolo, 2017).

When considering how social innovation is taught in business schools, social innovation is conceived and simplified as solutions for social problems, yet this definition often fails its potential because its success is measured in "effectiveness" and "efficiency" (Cairns, 2017). The foci in business schools are driven by management studies, which often derails the focus on human development (Cairns, 2017; Gözde et al,2017; Rogers, 2009). Such terms dilute and limit the application and capacity of social innovation rendering it as another commodified process. Social innovation is not intended to be an absolute resolution to the wickedness of social problems, nevertheless 
there a gains and benefits that come from its capacity to reframe an issue, if not already highlighting inequalities.

\subsection{Disability and Entrepreneurship}

According to Leah Lakshmi Piepzna-Samarasinha, the author of Care Work:

Dreaming Disability Justice, Disability is articulated as a "set of innovative, virtuosic skills" (pg. 126), adding "When I see disability justice flourishing, it comes from years if relationship building, and building trust, from fucking up, making repair, learnings from mistakes, and showing up for each other" (pg. 127). The use of personal identity become a site of manifesting political and influential drive; self-identifying as a femme, queer, disabled, sick and person of colour operates as a rally call for other marginalized identities. Much of Piepzna-Samarasinha approaches and criticism pay respect to Disability Justice Collective, a group of activists with a spectrum of intersecting sets of identities, whose membership also includes the previously mentioned Clare (2011), whom collectively, with its members, coined the term "Disability Justice" as a "movement building frame-work of intersectional, revolutionary disability politics" (pg. 122.) This approach is a type of politic that considers healing, revolutionary outcomes, and confronts shame through the process of unpacking histories. Piepzna-Samarasinha reminds communities that people with disabilities "are more than just their access needs" (pg.129) and that what abled bodied people describe as failures, are in fact expressions of Disability Justice. Its approach embraces the slow, and "when it's really happening, [Disability Justice] is too messy, and wild to fit into traditional movement and nonprofit industrial complex structures" (pg. 124). Additionally, "foundations have rarely ever given disabled people money to run our own shit. Nonprofits need us as clients and get 
nervous about us running the show. Disability justice means the show has to change- or get out of our way.” (pg.125). This unique approach to hack and circumvents challenges is an indicator that "Disabled people are experts and designers of everyday life. But we also harness technoscience for political action, refusing to comply with demands to cure, fix, or eliminate disability" (Hamraie and Fritsch, 2019; pg. 2).

On the other hand, when denied opportunities, entrepreneurship has been identified and studied as a path to inclusion for people with disabilities to participate in the labour market (Barclay et al, 2014; Shaheen, 2016; Caldwell et al., 2016; Maritz \& Laferriere, 2016). Inclusive entrepreneurship, as explained by Shaheen, (2016) can be located within the context of disability recovery and rights literature, which remain a stark contrast and opposition to critical disability claims from Hamraie and Fritsch (2019) regarding disability as a problem to be eliminated. Including in Shaheen's (2016) theoretical consideration are the negotiating process of agencies and state-supported programing. Within the context of disability, entrepreneurship can be considered an interchangeable term, with one focus on gainful employment granting themselves forms of income and the other focus concerns the creation of innovation (Maritz \& Laferriere, 2016).

High rates of failure in small business has been noted as a reason for discouragement for people with disabilities from pursuing entrepreneurship (Shaheen, 2016). Other barriers and deterrents include access to capital, and pre-conceived lower expectations of success from able bodied service providers (Caldwell et al., 2016). In contrast to the discouragements, the benefits of pursuing entrepreneurship have been noted to include "creative problem-solving skills, flexibility, grace under pressure, 
persistence, sense of humour, willingness to ask for help, and resourcefulness" (pg. 49) as suggested by Maritz \& Laferriere (2016). Entrepreneurship can foster community, connecting with other entrepreneurs, as a form of collective organizing and peer identification (Shaheen, 2016). Entrepreneurship has cultural and theorical connections to creativity, decision making and leadership (Fillis and Rentschler, 2010).

Entrepreneurship's innovative and creative ethos is almost recognizable as a form of Disability Justice, it shares concerns regarding political-economic and socio-cultural factors, particularly due to its ability to grant access and developing untapped potential (Harris, Renko \& Caldwell, 2014). Between disability and entrepreneurship, common ground can be noted, both are about survival, simply because it is not discussed does not mean it does not exist. Grook and Danbaar (2014) emphasizes this by opening their article with a quote from Anita Roddick (1942-2007), founder of the Body Shop, which states "Nobody talks of entrepreneurship as survival, but that's exactly what it is" (pg.17).

\subsection{Access, Discourse and Design Activism}

Understanding Access is a form of political discourse (Guffey, 2015). Elsharkway (2017) describes discourse in two areas; structure of the words themselves, and function of those words. Discourse analysis becomes needed for its "insight that text and talk play a key role in maintaining and legitimizing inequality, injustice, and oppression in society" (pg. 7) - designers can improve their capacities through understanding political discourse (Maun and Myhill, 2005) and more so through writing itself. Engaging in design criticism can improve the overall practice of design as suggested by Crisp (2009). Further, Thorpe (2011) argues in favor of understanding words because "another 
common way that design has taken up "activism" is through the notion of criticism and criticality" (pg. 3) and that "thinking through the definition of design as activism gives designers a language for communicating more broadly" (pg. 14). However, writing has its drawbacks, McRuer (2006) suggests that writing and composition, when focusing on queerness and disability, are sites of hegemonic practice, like Irving's (2009) “hegemonic bargaining," particularly in the arena of academia and education. Writing itself is a "practice that is focused on a fetishized final product" (McRuer, 2006;pg 151), as authorship and publish-driven interest are monitored, individuals and institutions are seeking a "return on their investments" (McRuer, 2006;pg 152). This includes queer and critical disability scholars, their collaborators, as well as universities themselves.

Through reconceptualizing one's own understanding of Access, designers can be more discerning when it is needed, especially when developing products and services (Guffey, 2015). Embracing “Access" as a multi-dimensional term would acknowledge the hostile terrain in which the word itself exist. Fritsch (2016), expands further on "Access" (pg. 23) by stating its purpose is associated to power, determining whom may and may not have "Access." Its application denotes both form of "contact" and "attack" to navigate such hostile terrains.

Discourse analysis when informed by critical disability studies can create a unique path to highlighting the promises and authorities that grant or deny "Access" (Phelan, Wright \& Gibson,2014). The Disability Rights Movement gained momentum by reframing attitudes of disability away from medical dimensions, refocusing disability as a societal issue. Design practice and education's focus on built environments, mainly physical, with less attention to the socially constructed, fails to reveal disability as a form 
of oppression (Guffey, 2015). Design, passively, or unknowingly undermines disability and people living with disabilities, by focusing on physical aspects of environments (Hamraie, 2016). Additionally, the capacity to "see" has historically been a form of "knowing," passively reinforcing that those who cannot "see" as a limitation, reminding that alternative ways of knowing are undesirable, defective or deficient (Featherman, 2016). Disability conflicts with design-thinking in the discourse of how new forms of knowledges are created. Any form of "empowerment" that relies on permission being granted or transferred refuses to understand that this transfer of "empowerment" itself exists due to sustained power imbalances. "Empowerment" is a performative empty gesture, focusing on individualism, rather than collective organizing (Riger, 2002; Hamraie, 2016).

Design Activism can be inferred as an extension of discourse analysis (Denison, 2011) particularly for its capacity to notice the unnoticeable. By knowingly responding to circumstances, design activism operates on self-awareness, incorporating a range of concepts, including sociology, economics and code of personal ethics (Julier,2013; Denison, 2011). Employed in unconventional ways, design activism is strategically executed to challenge communication barriers. Its presence and deployment can range from post-cancer dialogs (Butcher et al, 2016) to even small, quiet personal activities such as knitting (Hackney, 2013). A form of soft revolution, minor activities, often devalued activities, can become transformational of social relationships, becoming tactics and methods of reshaping, and affording agency in marginalize spaces, or lack of spaces (Hackney, 2013). Further, Design Activism operates with the intent or intervention to demand change within a system (Heidaripour and Forlano, 2018). Additionally, Design 
Activism is about a "counter-narrative" (Fuad-Luke, 2009; pg. 27) and can be utilized either knowingly or unknowingly.

Prior to digital spaces, Hal Fischer’s (1977) Gay Semiotics, a visual essay, can interpreted as critical contribution to queer and accessibility discourse. Its study, a series of photos, featuring queer men, and their strategies of engagement. On a surface level, the image and text inter-play to reveal men in various poses and artifacts, but collectively, it is the systems of communications that articulate a hidden existence of labor. More specifically, Fischer describes his work as a "lexicon of attraction" in an interview with Julia Bryan-Wilson (2015) for Aperture magazine.

Samples from this visual study, include a system of colour coded hanker chiefs, placement of earrings and key changes, and images of men in various archetypal characters such jocks, hippies and blue collared uniformed workers, and in Design may be referred to as personas. This contribution and imagery offer a critical essay and examination of life queer life prior to the HIV/AIDS crisis among the streets of San Francisco's Castro and Haight Ashbury districts. Parts of Fischer's interrogation on gay culture state:

In gay culture, the reverse is true. Signifiers exist for accessibility. Obviously, one reason behind this is that gays are less constrained by a type of code which defines people as property of others of feels the need to promote monogamy. The gay semiotic is far more sophisticated than straight sign language, because in gay culture, roles are not as clearly defined. On the street or in a bar it's impossible most of the time to determine a gay man's sexual preference either in terms of activity or passive/aggressive nature. Gays have many more sexual possibilities than straight people and therefore need a more intricate communication system...

...Like any other cultural group, gay people have developed a semiotics intended both for identification and/or invisibility within the larger culture, as well as communication among themselves. As economic, political and social levels of interaction fluctuate, the 
uses of the language will broaden and new, more evolved -- overt

as well as covert -- terms will come into use. As the gay community

is polarized on some issues and cohesive around others, the

semiotic process which helps locate in the larger culture will

flourish with the interesting and undoubtedly provocative results.

- Fischer, 1977 via QueerCultureCentre.Org

Its significance rest in its documentation of language, mainly non-verbal. This

documentation leaves evidence of a system, granting access, and moves through time for future generations to explore. The capturing of complexities, although tongue in cheek, underscores the importance of language and the development of words. Due to the lack of clarity, a system emerges. In many ways, this system of communication was response to "hostile terrain" (Fritsch, 2016; pg. 23) allowing queers to access communication activities between each other without repercussions of homophobia specifically this can be referred in Fischer's (1977) work regarding the "signifiers exist for accessibility." This essay and visual study can attest to the lives of queer people, demonstrating the capacity to operate simple yet complex discourses; always responding to political, social and economic circumstances, particularly when those circumstances indirectly or directly debilitate.

\subsubsection{Design Activism}

Julier's (2013) criticism and comments are notable among Design Activism literature, stating that design education is often devoid of neoliberal discussion within its education. More dimension is added by Çetin, \& Aryana's (2015) review of design activism and social responsibility design approaches on design educations, stating that it is not just devoid, it is insufficient. However, even before Julier, other critics of Design, in favor of Design Activism, included dissenting thinkers such as Victor Papenak (1971), 
known for citing that designers often create further problems than the ones they solve it solve. Other criticisms proclaim that "most things are not designed for the needs of people, but the needs of manufacturers to sell to people." (Papenak, 1983, pg. 46) More infamously, he is known for claiming that the only thing phonier or more damaging than industrial design is advertising. In the defense of advertising (and marketing) Murray, Evers and Janda (1995) highlight the importance of theory borrowing, a concept exercised within advertising and marketing, stating: "researchers should not borrow only those theories that are consistent with existing research streams in marketing; this would merely reinforce what already has been learned" (pg. 104). More importantly, this approach of theory borrowing in rooted in emancipatory interest. This process of theory borrowing has been historical demonstrated by ACT UP (Heller, 2013) through the repurposing of advertising into activism and reclaim public spaces into protest. Other cultural figures include Kalle Lasn's (2006), made famous from Adbusters, a magazine dedicated to critiquing capitalism through advertising. Lasn (2006) shares the concept of "Design Anarchy" stating that the "aim is to topple existing power structures and change the way we live in the twenty-first century" (Armstrong, 2009, pg. 107). Design Activism needs to borrow further theories to gain a deeper philosophical grounding, (Song \& Lou, 2016) while also accepting its gripping role to accessing the past, its histories and its ability reform the present, thus informing the future (Lees-Maffei, 2011).

Returning to Papanek, Clarke (2013) has argued that Papanek's work has been known to be a "design polemic of its time, politicizing a generation of design students" (pg. 153). Further, the emergence of social responsibility and participatory design has roots in Nordic and Scandinavian Design; described as noble for its deep consideration 
for social well-being. These perspectives have led to a shift in the design paradigm to focus on processes of collaboration which were engaged, and action focused, which can be further attributed by designers and dissenting concerns (Guffery, 2015; Lie, 2016). Other dissenting concerns come from individuals such as Tony Fry's (2009) process of futuring of design, and that designers are "de-future"-ing; meaning if design continues to practice as it does, design will render itself useless as we compromise the entire planet and its resources; using bio-diversity as a metaphor, design as a monoculture can carry "a high risk of extinction"(2009,pg 92). Marshall-Baker's (2011) comments expand Fry's argument reinforcing that current practices are, regardless of their intent of making ecofriendly products, that these eco-friendly products, too, are just as dangerous. To effectively improve design, design must apply itself outside of design. In other words, design activism must be better understood and studied to grasp how design can respond, alter, and even take advantage of neoliberal institutions, resources and structures (Julier, 2013).

Designers and Academics are skilled in appropriating and defining the occurrences of a movement, regardless of its political nature. In this process, it may express itself in many forms, such as a product, service, publication or new form of knowledge. The impact of such production may not always lead to substantive changes which is often associated with design. As a result, power imbalances are sustained, leaving a trail of acquired cultural capital through means of empty gestures. (Shultz et al, 2018) Concepts such as Decolonization are performed without sincere intent and show the potential of being reconfigured, similarly, to terms such as "Sustainability" as an accomplice to neoliberal forms of design (Shultz et al, 2018). To effectively understand 
Decolonization, Shultz et al, (2018) borrows from Escobar (2015), acceptance of social messiness as a needed approach for understanding the different ways of knowing in the world. By mapping out diverse forms of knowledge, it recognizes what Julier (2013) argues for design's role in occupying new intellectual territory.

\subsection{Education and Design}

Dimarco (2011) suggest that the future design education can benefit from "Issue Based" methodologies, as it will allow designers to appreciate the social context. This argument is made by draw on discourse of design scholars who have argued "that designers need to be more socially conscious and socially relevant when creating visual products" (pg. 73) as "most communication design educators do not emphasize political or social issues in their teaching." (pg. 74). Understanding social impact and relevance must go beyond the visual components of products. At its core, future design education needs to elevate its communicative capacities and consider inclusive approaches.

Strategies for teaching inclusion in academic design curriculum may consider developing in-house case studies, small scale projects, reduced class size to invite external participants, and to learn more about the complexities of ethics procedures (Dong, 2010). Furthermore, improving cultural competencies and developing an intercultural awareness on a personal level has been argued to be beneficial to design-asa-practice and design education ( MacLeod and Macdonald, 2016; Mendoza and Matyók, 2013). The process to improve design through education needs to move beyond just introductory exposure to concerns; cultural immersion facilitates the elevation of design's social contributions and impact (Mendoza and Matyók, 2013). Students' lack experience and perspective on complex issues may prevent design education from improving. Access 
and exploration of complex issues can be done through means of volunteer work (MacLeod and Macdonald, 2016). Engaged social methodologies in design would result in transformational experiences contributing to a holistic understanding of citizenship (Mendoza and Matyók, 2013; Matyók, Mendoza, \& Schmitz, 2014). Engaged social methodologies in design often involve designing for people other than oneself. As a result, design curricular content has begun reaching towards other disciplines such as anthropology and sociology to bridge cultures and to redefine itself (Buck-Coleman, McDonald \& Bidle, 2011).

Design education continues to expand and explore other intellectual territories (Julier, 2013). One of its notable expansions is towards the concerns of Access and Accessibility. Often this is done through the means of Universal Design. The practice of universal design exists in mixed contributing systems, informed by institutions and locations. The crossing between these spaces results in transformational impact on design knowledge itself and its practice. "Design and dis/ability are co-constituting" (pg. 1317); seeking to design for diverse bodily needs must be practiced with the acceptance "that there is never any settlement or finality about what will work or not for different people" (Imrie and Luck,2014; pg. 1317).

Hamraie (2016) argues that [Universal] Design is not immune to implicit bias. Further critical observations underscore the condescending and demeaning tone of Universal Design. Claims of thoughtfulness are co-opted into a codified process to allow Design to be positioned as more commercially desirable and morally acceptable. Through this process, the elimination of barriers is synonymous with erasure of disability. Attuning to Kafer, (2013) a feminist queer Crip perspective, Hamraie (2016) highlights 
that the Design often overlooks disability, deems it an afterthought, and fails to acknowledge disability as a path for new understanding and discovery, and that "life with disability is begging for corrections" (pg. 301). Assuming Universal Design as the panacea to disability, implicitly reinforces pejorative ideas of disability — it is very much an insult to the complexity of the world. When Universal Design is passively practiced as a prescriptive checklist, it undermines disability as an equal contributor for alternative ways of knowing. Universal Design lacks the insights of critical disability theory (Hamraie, 2016). Further, design can advance its capacities by adopting the words of Clare (2011), rejecting and disassociating "inability with disability" (pg. 37). More specifically, access is be better informed when understood for being collective, messy, experimental, frictional, and generative (Hamraie and Fritsch, 2019).

The evolutionary development of design education implies an acquisitional approach (Buck-Coleman, McDonald \& Bidle, 2011,) which suggest evidence against design, counter operating towards decolonization (Shultz et al, 2018). This acquisitional approach and expansion of intellectual territory, foreshadows, what may occur to knowledge produced from design when informed by Critical Disability Studies, serving as a reminder than design is not immune to neoliberalism (Julier, 2013) thus indicative of Design Activism's vulnerability to being coopted. Critical Disability Studies, like Design Activism, and Design as a whole field of study, can potentially be easily manipulated according to any political will. Further implications involve design education developing a more nuanced understanding of "intention and reception towards ends that are responsible in a societal context" pg.12 (Buck-Coleman, McDonald \& Bidle, 2011) and must acknowledge the limitation of its assumed neutrality (Imrie and Luck, 2014). 
Disability scholar, Stella Young, has provided a term which captures the performative and empty gestures which Haramraie (2016) critiques, this can be best described as "Inspiration Porn" (Young, 2014), meaning, the notion of feeling good, outweighs the importance of doing good.

\subsection{Design's Directions}

The concept of direction, orientation and disorientation by known design intellectuals is expansive. Design thinkers such as Papanek (1971) have argued for the proliferation of design, liberating design from itself, and industrial design, as it's known today, needs to cease to exist; Thorpe (2011) has argued in favour of champing design to benefit those most neglected in society, while, Markussen (2013) critiques Thorpe for limiting their scope and over emphasizing communication and protest; Grudin, (2010) proclaims that design at its best, operates unnoticed, while Bonsiepe's (2006) claims "the designer's task consist of making [these] invisible functions visible" (pg. 33). However, it is Buchanan (1992) who reminds academics and designers that "there is no reason to believe that parts and wholes must be treated in ascending rather than descending order." (pg.10). His ideas are recognizable with a subtle trace, ode, awareness and appreciation of disability and queerness, particularly, when claiming that "parts and whole are of many types and may be defined in many ways." (pg.10), meaning design itself is a rather fluid process and non-static in orientation. Designers are socially located and equipped with power, more importantly, it is a power that demands responsibility with a preparedness to support communities and society as suggested by Buck-Coleman, McDonald \& Bidle (2011). Changes in Design Activism will occur with the changes in research directions, particularly when research shifts away from a publications-driven 
agenda (Song \& Lou, 2016). From a feminist's futurist orientation, design activism may need to take new forms by presenting beyond scholars and policymakers; Heidaripour and Forlano (2018) argue further by highlighting that the aim is to transform participants of design activism into agents of change.

In Steven Heller and Veronique Vienne's Citizen Designer: Perspectives on Design Responsibility (2003), three categories of responsibility emerge, social, professional and artistic. It makes a subtle hint that responsibility is a mixture and balancing of all three. Among the essays, Maud Lavin's Who Gets to Say What to Whom? focuses on tone as form of direction, and whom it might need directing towards. More specifically, "design criticism itself needs to [be] editorializing and polemical" (pg. 109). In its second edition (2018), it further expands of issue around race, unpaid labor and design's occupancy in neoliberalism. Contributor, Dr. Miriam M. Ahmed (pg. 104) draws attention to the above-mentioned ideas, by identifying practitioners of Design Activism as "minority designers", individuals who use, and operate outside the mainstream industries of commercial design. Much of what Dr. Miriam M. Ahmed indicates can be even further supported and illuminated by Thorpe (2011) who states that “good design (usable, profitable, beautiful, meaningful) doesn't usually constitute activism on behalf of excluded or neglected groups.” (pg. 1)

Outside of this anthology, many of its ideas echo what is suggested by Imrie and Luck, (2014) that Design by extension, is a driver for social and technological change, and is, by default never neutral; design is politically charged whether explicitly stated or not. This is because Design consist of "intention and action towards a desirable end" (pg.12) as argued by Buck-Coleman, McDonald \& Bidle (2011) further adding that, 
adopting awareness of other designers within their community is needed as practices can be shared among designers. Outside of the design profession, concepts such as designthinking have been adopted, notably within the area of business (Verganti, 2009; Martin, 2009; Brown,2009, Kimbell, 2012) The adoption of design outside of design should be no surprise considering its role to facilitate and navigate complex systems (Buchanan, 1992). More importantly, it is design's role to reduce discomfort and ambiguity, creating confidence for diversity in discussion thus leading to better solutions. (Liedtka, Salzman \& Azer, 2017).

\section{Chapter 3: Method}

\subsection{Approach}

The goal of this thesis is to investigate Design Activism within the context of LGBTQ communities, with a focus on invisible disabilities and episodic conditions; conditions which cannot be visible detected or conditions that occur in unscheduled fluctuation (Vick, 2012; The Episodic Disability Network, 2015). The setting of social and community health services provides a site of study for using design research methods and participatory practices. This provides a common entry point to the LGBTQ communities. Given the complexity of these environments, the methods utilized aim to understand how knowledge is socially constructed by the environments and social roles, thus the methodological approach of this study aimed to be grounded in an action and practiced-oriented framework. In some quasi fashion, this study calls up the ethos of, Latour \& Woolgar's (1986) Laboratory Life, yet, the only difference is that researcher is already submersed within Disabled LGBTQ culture and social service environments. Through these methods, an exploration between the social and the technical pursue an 
understanding of contradistinguish through a means of triangulation. The use of qualitative methods was chosen over quantitative methods but sought to seek a balance between both. The use of qualitative methods was chosen as they would provide a richer context to illuminate the complexities of LGBTQ communities. This chapter discusses the process of each method and context of settings, and protocol for collection and how the analysis was intended to address the following question, and its sub-questions.

Q1: What is Design Activism in the context of LGBTQ communities, living with invisible disabilities and episodic conditions?

SQ1: What is Design Activism relationship to Social Innovation, in the context of LGBTQ communities, living with invisible disabilities and episodic conditions?

SQ2: What barriers or limits exists that result in the discouragement of Design-Activism?

SQ3: How might Critical Disability Studies and Queer Theory inform Design Activism as a method /practice?

\subsection{Pre-planning, Concerns and Training}

Prior to engaging and pursuing graduate studies, the researcher had been developing various design-research projects within their respective communities. Through these projects the researcher has developed an inherent experimental ethos, which leans towards action-based possibilities. During this study, the researcher had the privilege to travel, and present at notable conferences, exercising their presentation skills, as well as attending valuable sessions regarding sex, gender and sustainability. These experiences have contributed to prototyping, reformulating and iterating this thesis study's questions at large. A mix of the fore mentioned experiences have been vital towards shaping this research study, yet, contribute to biasing the study with a level of militant optimism and tongue-tied pessimism which sets the tone of the research itself. 


\subsubsection{Target Sample Population}

Non-profit and Social Service agencies were the entry point to engaging LGBTQ communities due to concerns of risks and aversion of coercion. Notable public facing LGBTQ organizations that fit the inclusion criteria were emailed using a scripted email (See Appendix F). Most of this segment's participation came from Ottawa and Toronto. However, the over laying criteria needed was a form of self-identification with LGBTQ communities and an understanding of disability, either from lived experience or professional engagement. Verbally shared, all participants were informed by the researcher that they too, identify as an LGBTQ community member living with multiple invisible disabilities. This voluntary disclosure was done for the purpose of developing trust with participants, as well as to demonstrate that the knowledge being created was done in a "nothing about us, without us" ethos.

\subsubsection{Recruitment Procedure and Population Targeting}

Links for the online data collection forms (See Appendix C and E) and researcher contact details were sent directly to service provider agencies and non-profit who matched the inclusion criteria, specifically: be $18+$ years of age; residing within Ontario, Canada; and must self-identify with the LGBTQ+ communities. Must either be living with an invisible/episodic disability or volunteer/work in a professional capacity as a service provider for these respective communities. Participation is voluntary.

Given the researcher's community affiliation, the ethics board had imposed that the researcher limits their distribution as a mitigation strategy to avoid coercion from the researcher's existing peer and social circle. Social service agencies within the Ottawa and Toronto area were identified and then sent the digital links, along with an 
explanatory email regarding (See Appendix C, E, and F) the intent and purpose of study. Included within each method were its tasks involved, the time commitment and potential risks. Voluntary participation was highlighted frequently among the recruitment materials.

Service Providers were targeted with a trickle-down and cascading strategy, assuming the agencies would potentially forward the researcher's digital forms and contact details to their communication channels. As a result, it was assumed that potential LGBTQ people would either follow up directly with the researcher or simply part take within the online form. Online form respondents were given the opportunities to identify as either a service provider or service user. Under the study's inclusion criteria, participants must affiliate to the LGBTQ communities and living with an invisible disability, episodic disability, or provide services to these respective communities. Under no circumstances were participants asked to disclose their medical conditions or disability. Additionally, should participants wish to participate further, an additional question was added within the terms and definition online form, allowing participants to provide their contact information. An additional recruitment question was only added towards the terms and definition form due to the researcher deeming it the lowest of time commitments, as the other two methods involved higher forms of creative commitment. Upon submission of the digital forms, both in the terms and definition form and photovoice activity, a "Thank You" message appeared. It followed asking participants if they knew of any other individuals potentially interested. Following the recruitment prompt, short debriefing acknowledged the risk and concerns regarding being emotional or mentally distressed. If the situation deemed necessary, participants were informed to 
reach out to the researcher to request a list of local resources.

The strategy for recruitment was originally conceptualized as a sniper approach, meaning publicly known agencies would be targeted. This would involve sending a series of emails to openly known LGBTQ affiliated agencies. Considering the researcher's working history involved with LGBTQ organizations, completing an Ontario based list was simple, and existed within the researcher's resources. Many of these contacts were a mix of personal and professional relations. Recipients were sent a scripted email (See Appendix F) with modification according to their inclusion criteria. Given the nature of LGBTQ community work, the luxury of divorcing personal and professional relationships may not always exist; the researcher asserts that this circumstance is a double-edge sword, and overall this may have worked against the researcher's advantage.

\subsection{Method 1 - Terms and Definition Online Questionnaire Form}

The use of a digital and online form was intended to allow for a greater reach with the broader LGBTQ communities, while also allowing participants to share the survey among their peers. The use of the digital form was conceptualized as a tool that would be at ease for both participants and researcher. The focus of this methods aimed to understand the confidence level and knowledge of commonly used terms at the intersection of Design, LGBTQ communities, Disability and Social Innovation. Literature (Miller, 2017; Heller, 2013, Thorpe, 2011, Fishcher,1977; Elsharkway, 2017; Crisp 2009) has argued that language and words are a site of contention. The selected words, (Appendix, C) were chosen given their prominence in literature (Thorpe, 2011, Julier, 2013, Fuad-Luke, 2009) and their loose application according to the researcher's 
understanding and personal practice.

The first set of questions focused on collecting demographic markers to allow the researcher to categories participant submission between service providers and service users. Next, the question focused on familiarity of terms and definition. These questions were broken into two parts. The first question asked for participants to rate their understanding of a term on a scale of 0-to-100. Secondly, participants would be asked to share their interpretation of the term. A total of 10 terms were investigated (See Appendix C), followed by questions requesting to share under and overrepresented concerns within the participants' LGBTQ communities. Lastly, the final question involved asking if participants wished to be involved further within the study. For the data to be successfully collected, the form required participants to confirm, and consent one more time by hitting the "Submit" button. No data was collected from incomplete forms, or browsers which were closed part way through.

\subsubsection{Setting - Context}

The use of digital online form was intended as an outreach strategy. Also, the use of a digital online form would reduce the number of physical interactions with the researcher with LGBTQ people, as it were assumed that the researcher would impose their own bias on participants. Additionally, the use of a digital form allowed for easier sharing between participants and peers, as well as a reduce transcription time. This form and methods could have potentially been done in person, but to reduce and mitigate risks, whether perceived or real, the researcher adopted the ethic board's suggestion, and distance themselves from direct interaction with study participants. Furthermore, the use of a digital tool allowed for easy data collection and reduced transcription time, as 
content can be simply copy, pasted and coded.

Additionally, the focus on words was a direct result from the finding within the literature. Studies, comments and critics of language (Fredriksen-Goldsen,2016; Fritsch, 2018; Fishcher,1977; Elsharkway, 2017; Crisp 2009; Thorpe, 2011)

\subsubsection{Data Analysis and Examination}

The given the intent to use mix methods, one part of this methods involved assigning a statistical variable of the participants perceived understanding of a term. More specifically targeting participant's confidence level, using a scale of 0-100, 0 being an absence or lack of knowledge of term and 100 being an absolute understanding of the term; 50 was used as an indicator of awareness of the mentioned term, but an awareness of uncertainty. By assigning numerical values, this study can either tally total scores and compare averages or differences between averages.

\subsection{Method 2 - Card-Sorting and Semi-Structured Interviews}

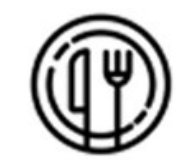

Food

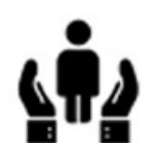

Social Worker

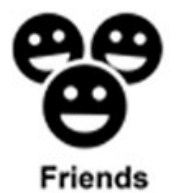

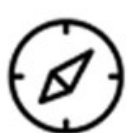

Travel

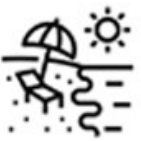

Leisure

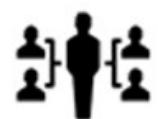

Co-Workers

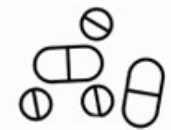

Medication

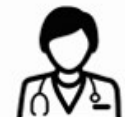

Family Doctor

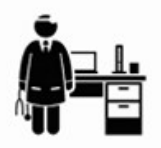

Medical Specialist

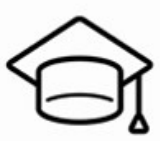

School

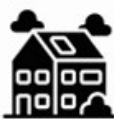

Housing

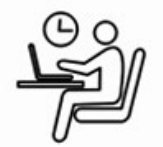

Employment

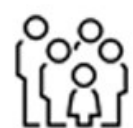

Family

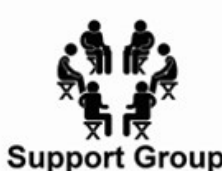

Support Group

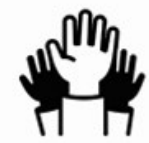

Volunteering

Figure 1.1 Card-Sorting Activity Concepts 
The activity for this segment involved providing participants with a deck of cards with the task of organizing them. The cards created for this activity consisted of 15 concepts shown above.

These concepts where determined through casual conversation with LGBTQ people living with disabilities and the researcher, specifically those who are accessing the provincial disability support program, ODSP (Ontario Disability Support Program). Additionally, this deck was tested with people living with chronic and episodic conditions, prior to its submission to the ethics board. None their data used within the final study. Their participation and consultations and contribution were part of the codesign process (Sanders and Stappers, 2008) to determine the feasibility, navigation, process of development and research scripts.

As part of this activity, participants would be then asked to organize the deck according to what they believe to be a matter of most importance and priority. This activity was conducted with a script (See Appendix B) with a prompt focusing on postdiagnoses of an invisible/episodic disability scenario. This segment investigates the participant's application of tacit knowledge of navigating and accessing services, while also highlighting their decision-making process. This activity of sorting cards involved both a text and image-based stimuli. Participants were provided additional blank cards and a pen should they wish to add more concepts to the deck, as well, they had the option to remove cards too. Upon completing and finalizing their organized set of cards, participants were asked questions in the form of a semi-structured interview, a mix of set questions (See Appendix B). Sessions were to last roughly 20-60 minutes. 


\subsubsection{Setting - Context}

Participation within this segment relied on study candidates reaching out towards the researcher to schedule time and date. Upon confirmation of time and date, participants were provided a consent form regarding the tasks and purpose of the study in advance. Upon arrival for the session, participants were given a printed consent form (See Appendix C) for which they could sign. This process was done in order to ensure participants had enough time to consider their involvement. The option to have a nonvideo recorded option was available, as participants were ensured that no punitive or reprimanding had any participants choose to opt out of being documented. Alternatively, the participants were informed and were orally asked if the researcher could take handwritten notes.

Additionally, participants were informed that they can choose to have certain response removed from the study, should they wish. Upon completing of the session, participants were reminded that they had up to two weeks to withdraw their data, as this method was the only activity which allowed the researcher to identify responses to participants. All session occurred in a private room with the recording camera's tilted towards the sorting cards.

\subsubsection{Data Analysis and Examination}

This segment captures two key forms of data, the organization of artifacts, as well as verbal articulation regarding their process. These sets of data were collected with the intent to compare between each other, in the categories of service users, service providers and then finally as a collective comparison.

From these activities, we can determine how data is visually expressed differently 
between participants, as well as factor various external concerns which may not be so notable on the surface. This activity, compared to the previous method for the terms and definitions, focuses solely on qualitative data. While, the methods aim at the collection of qualitative data, the method itself can be utilized to document and capture number of instances and occurrences, as a means of generating quantitative data.

Upon completion of sessions, after two weeks, data and video content were transcribed and coded. Audio data was deleted immediately after transcriptions. From the video, only the final organized deck of cards was saved for the final study. The use of video was employed, originally with the intent of tracking movement organizing. Later the research asserted that studying movement may move beyond the scope of the study. The use of video was determined as a technical solution for not needing multiple devices. The findings from the interviews would eventually be reviewed, and revisited from the context of a Strengthen, Weakness, Opportunities and Threats (SWOT) analysis and thematically observed.

\subsection{Method 3 - Photo-Voice / Photo Journaling}

This method follows studies (Holtby et al. 2015; Mitchell, De Lange, \& Nguyen, 2016; Bardhoshi et al, 2018) as their use of imagery allow for deeper and richer insight into the lives of its participants. This method relies on participants having and owning a smart phone device to which they can capture their own personal images for the study. Participants were asked to focus on places and objects. Any submission of people within the photos, relied and assumed participants acquired consent and hold ownership of the image. Specifically, for this segment, participants were asked to focus on places of inclusion and exclusion. The maximum allowance of submitted images were a total of 
five images, with the minimum being one image.

\subsubsection{Setting - Context}

Originally, the option to allow participants to have an in-person session was proposed. Given the limited time and level or perceived risk with LGBTQ communities, the researcher was strongly encouraged to remember to limit and mitigate any risk. Thus, the option of a digital platform, similar the terms and definitions method were conceived. This activity could occur anywhere the participant wish to take the activity. Participation within this session was conducted completely anonymously. However, participants were given the option to provide a pseudonym for which the photo could be credited within the final study. Alternatively, participants that did not provided a pseudonym, the researcher would provide one in the absence.

\subsubsection{Data Analysis and Examination}

Generating a content map (Witter-Merithew, n.d) through a line-by-line review of the text will be explored as a strategy to develop visual tool for visual-spatial representation. From this map, thematic connections can be made about the relationship between inclusion and exclusion and between each participant. 


\section{Chapter 4: Findings}

\subsection{Terms and Definitions Online Questionnaire Form}

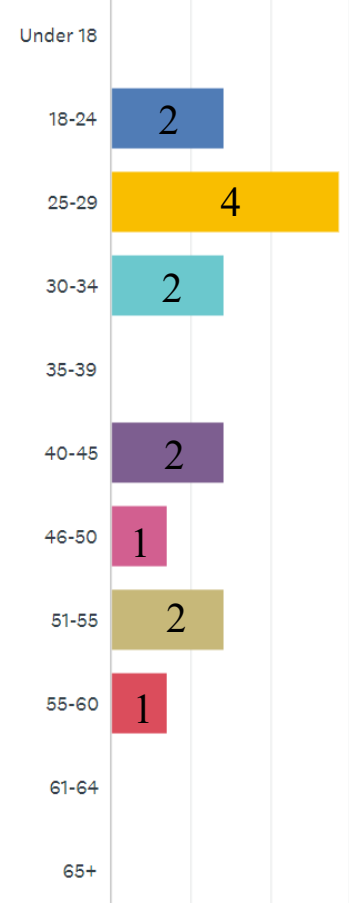

Figure 1.2 Age of participants from Terms and Definition Participants.

Finding: On a highly surface level observation, what can be easily deduced are two age groups that exist within this segment. The age categories exist as: Millennials and Boomers. For this segment there is a total of 14 submission. Considering the sample size, not much can be asserted regarding age as a factor, however, age my play a different role in other parts of this study. 


\section{SERVICE USERS: $\square \square \square \square \square$ TOTAL OF 7}

LIKERT-TYPE SCALE FOR FAMILIARITY

\begin{tabular}{|c|c|c|c|c|c|}
\hline TERMS AND DEFINTIONS & $\begin{array}{c}1 \\
\text { NOT AT ALL } \\
\text { FAMILIAR }\end{array}$ & $\begin{array}{c}2 \\
\text { SLIGHTLY } \\
\text { FAMILIAR }\end{array}$ & $\begin{array}{c}3 \\
\text { SOMEWHAT } \\
\text { FAMILIAR }\end{array}$ & $\begin{array}{c}4 \\
\text { MODERATELY } \\
\text { FAMILIAR }\end{array}$ & $\begin{array}{c}\mathbf{5} \\
\substack{\text { EXTREMELY } \\
\text { FAMILIAR }}\end{array}$ \\
\hline DESIGN THINKING & $=$ & & $= \pm=$ & & 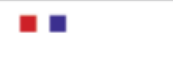 \\
\hline DESIGN ACTIVISM & $\|=$ & \pm & $=$ & & 1. \\
\hline ACTIVISM & & & & - & $=$ \\
\hline SOCIAL INNOVATION & " & & $m=$ & & 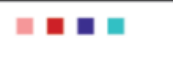 \\
\hline SOCIAL ENTERPRISE & 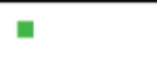 & $=$ & $\|=$ & & 10 \\
\hline DISABILITY & & & & 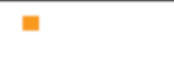 & " - " " = \\
\hline EPISODIC DISABILITY & & 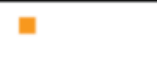 & $=$ & $=$ & $m= \pm$ \\
\hline INVISIBLE DISABILITY & & & $=$ & $=$ & " = " = \\
\hline ACCESSIBILITY & & & & & "E \\
\hline ODSP & & & & & 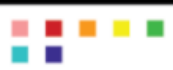 \\
\hline
\end{tabular}

Figure 1.3 Service User Familiarity - Likert-Type Scale 


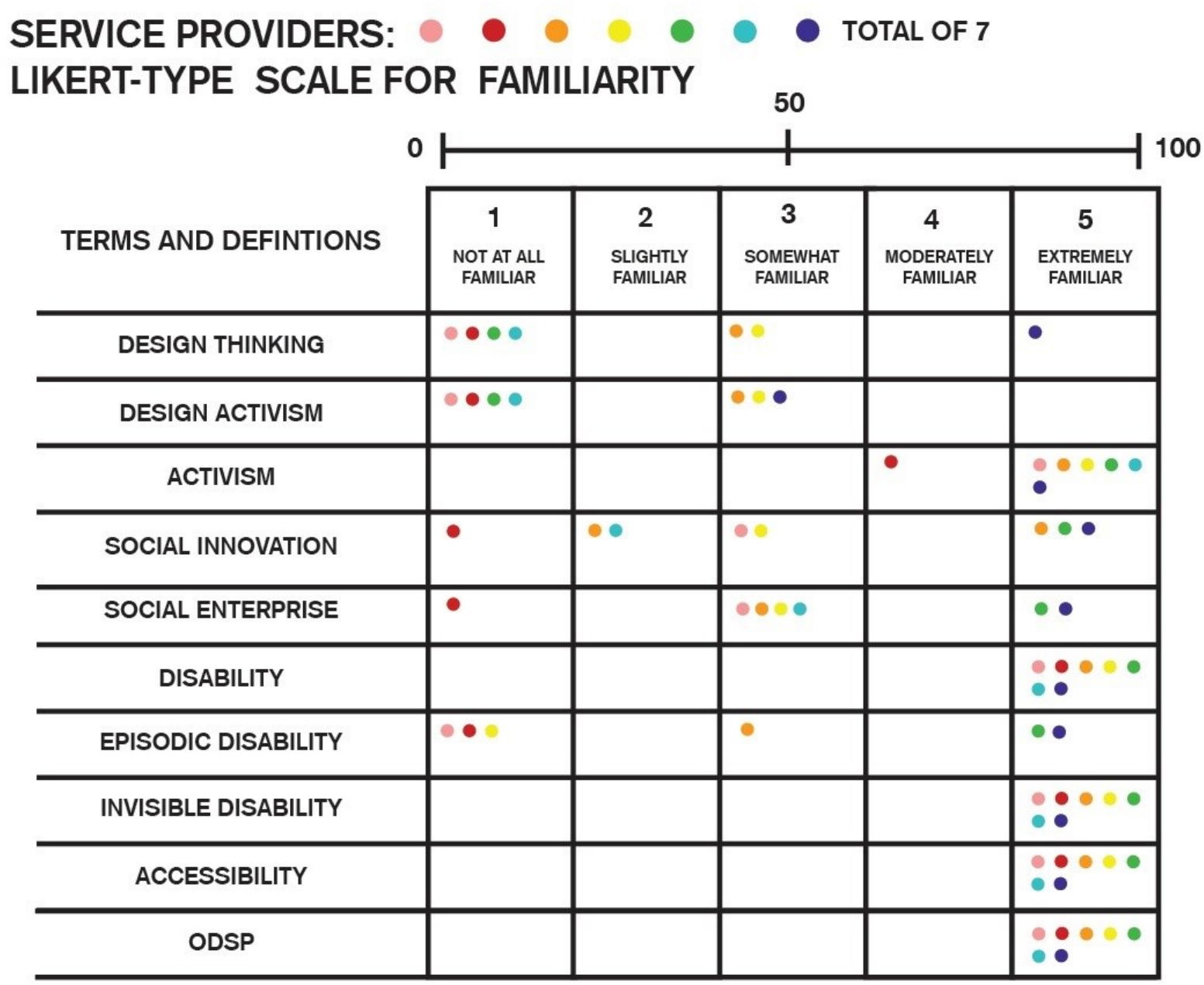

Figure 1.4 Service Provider Familiarity - Likert-Type Scale

Finding: Self-reported with the highest familiarity, between both Service Providers and Service Users are: Disability, Accessibility, Activism, Episodic Conditions. Terms such as Design-Thinking and Design Activism were reported with lower self-reported familiarity. 


\begin{tabular}{|c|c|c|c|c|c|c|c|c|c|}
\hline design_thinking & design_activism & activism & social_innovation social_enterprise & disability & episodic_disabilit! & abilit! & & ODSP & service_provider \\
\hline 0 & 0 & 100 & 90 & 50 & 0 & 0 & 90 & 90 & 0 \\
\hline 0 & 2 & 100 & 50 & 90 & 1 & 100 & 100 & 89 & 1 \\
\hline 20 & 0 & 66 & 18 & 78 & 19 & 80 & 83 & 14 & 1 \\
\hline 50 & 49 & 90 & 89 & 93 & 41 & 94 & 89 & 98 & 1 \\
\hline 93 & 99 & 97 & 100 & 100 & 100 & 100 & 97 & 100 & 0 \\
\hline 50 & 22 & 98 & 50 & 68 & 25 & 89 & 83 & 98 & 0 \\
\hline 4 & 7 & 71 & 45 & 73 & 9 & 75 & 76 & 81 & 1 \\
\hline 50 & 50 & 90 & 66 & 100 & 100 & 100 & 100 & 100 & 0 \\
\hline 51 & 0 & 100 & 75 & 100 & 100 & 99 & 97 & 100 & 1 \\
\hline 0 & 0 & 100 & 26 & 100 & 51 & 100 & 100 & 100 & 1 \\
\hline 0 & 0 & 79 & 0 & 100 & 80 & 75 & 100 & 100 & 0 \\
\hline 0 & 0 & 100 & 99 & 100 & 100 & 100 & 100 & 100 & 0 \\
\hline 100 & 97 & 84 & 89 & 88 & 86 & 77 & 100 & 100 & 0 \\
\hline 11 & 10 & 90 & 85 & 95 & 98 & 98 & 98 & 98 & 1 \\
\hline
\end{tabular}

Table 1. Self-Reported Familiarity of Term.

Finding: The following table highlights the data collected from the self-reported familiarity scales. Service Providers are highlighted in yellow, in the table below, however, for software application, a coding system of, $1=$ Yes and $0=$ No, were used to identify Service Providers and Users. This table was then used to create the Scree Plot Graph.

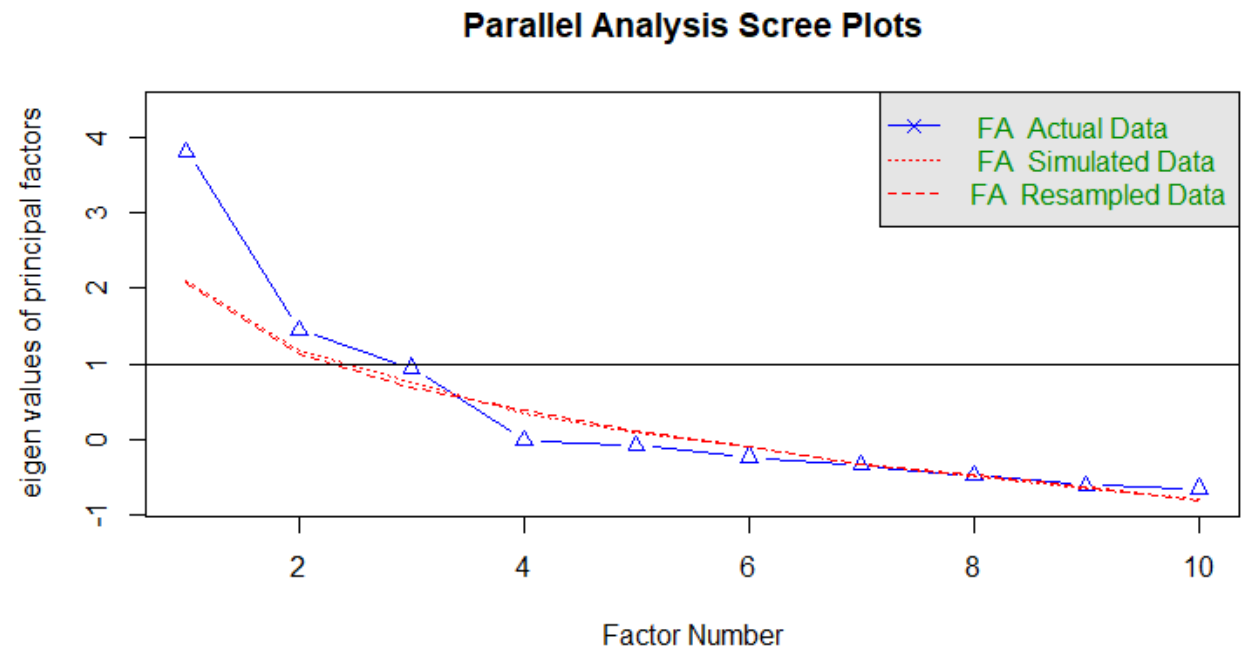

Figure 1.5 - Sree Plot Graph 


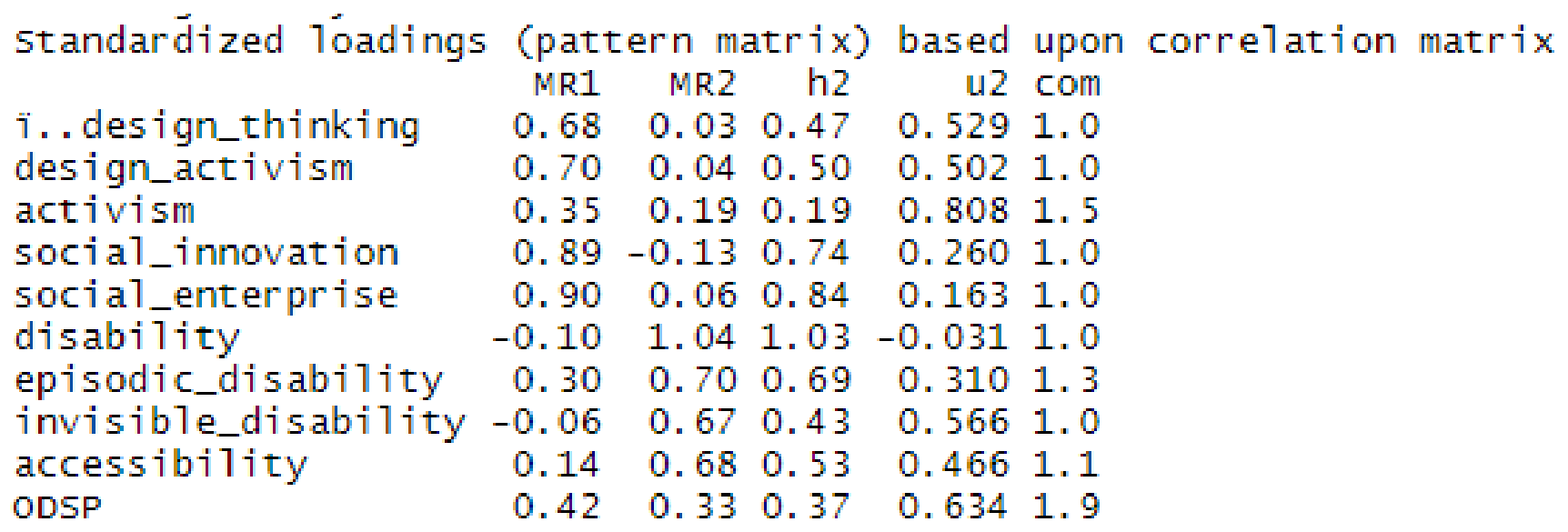

Table. 2 - Factor Analysis Results MR1 and MR2

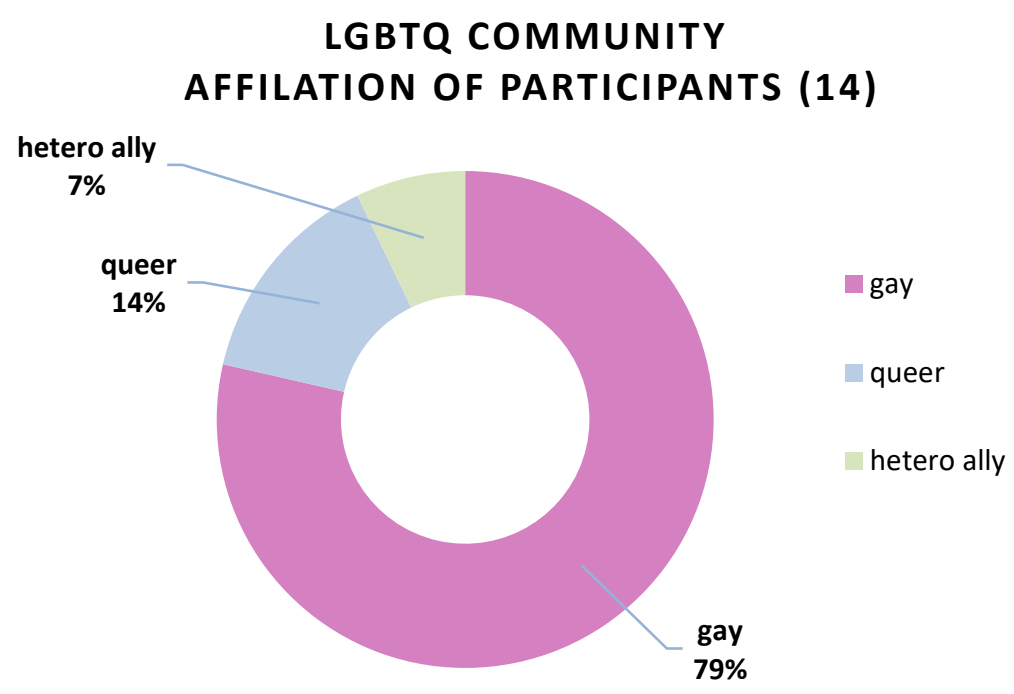

Figure 1.6 LGBTQ Community Affiliation of Participants

Finding: Most of the participants identity as gay male, with a total of 11 of the 14 . The other two categories included Hetero - Ally, with a total of one, and the remaining two as Queer. Within the category of Gay, the following table is break down of additional provided information. 


\begin{tabular}{|c|c|c|c|}
\hline $\begin{array}{c}\text { Male: } \\
8\end{array}$ & $\begin{array}{c}\text { Trans, Man: } \\
1\end{array}$ & $\begin{array}{c}\text { Male, Person of } \\
\text { Colour: }\end{array}$ & $\begin{array}{c}\text { PHA (Person Living } \\
\text { with HIV/AIDS), } \\
\text { Indigenous: } \\
\end{array}$ \\
& & 1 & 1 \\
& & & \\
\hline
\end{tabular}

Table 3. Gay Sub-Categories

\section{WHAT DO YOU BELIEVE ARE THE MOST CONCERNING ISSUES?}

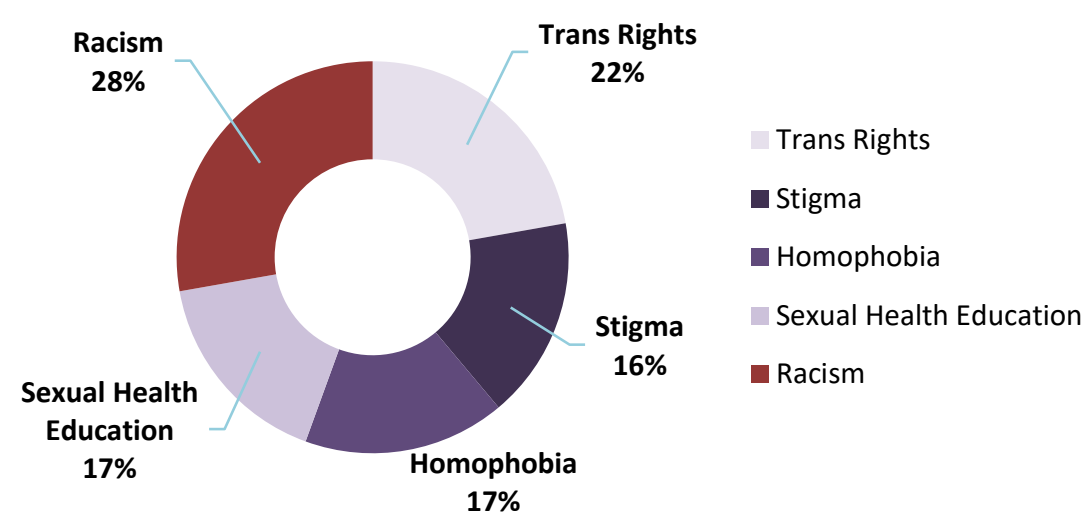

Figure 1.7-Q23 from Terms and Definition Questionnaire

\section{WHAT ISSUES DO YOU BELIEVE ARE NOT GETTING ENOUGH ATTENTION?}

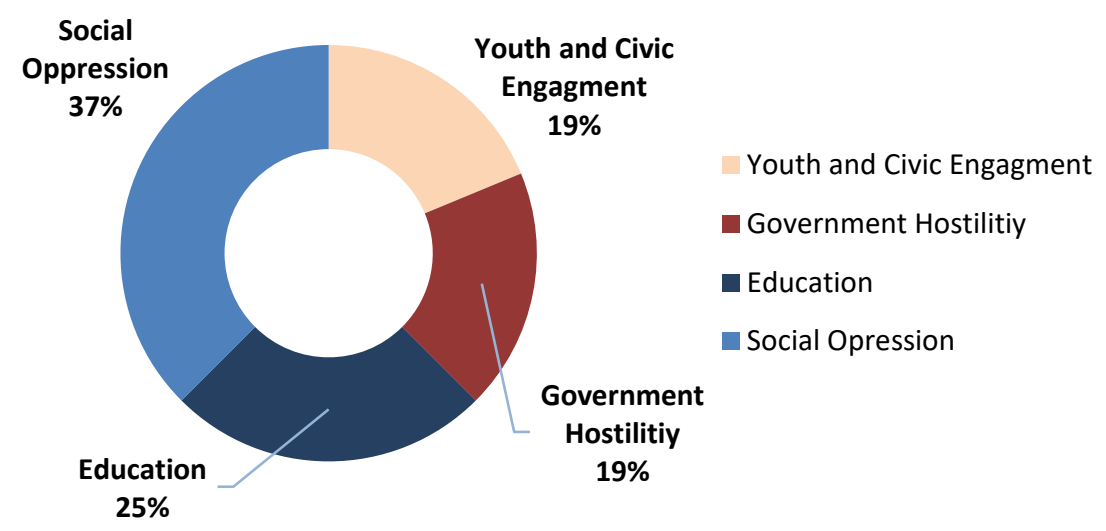

Figure 1.8-Q25 from Terms and Definition Questionnaire 
Finding: Within the study sample, Racism leads as the most self-reported concern and issue facing LGBTQ people in Ontario, Canada. Comparatively, Social Oppression leads as the most self-reported concern and issues which the sample believes to not getting enough attention in Ontario, Canada. Between Figures 1.4 and 1.5, the terms and categories within the donut chart are presented as submitted by the participants. These donut charts are representatively of a total of 14 service users and providers.

\subsubsection{Categorizing Data}

In total, the terms and definition segment attracted a total of 14 participants. To be more effectively discerning with the data, two Likert-type scales were created. With these two Likert-type scales, comparison between service providers and service users became more apparent. Considering the use of a sliding bar, respondents had a range of response options, ranging from 0 to 100 . Given such a wide range, the researcher had implemented and outsourced the logic of the data categorization to a Likert-type scale of familiarity (Vagias, 2006).

\subsubsection{Comparing Service Users and Providers}

From the data outsourced in a Likert-type scale for familiarity, it comes as no surprise that terms such as: Disability, Activism, ODSP, Episodic Disability, Invisible Disability, Accessibility indicate higher self-reported knowledge. Subsequently, the other terms, Design-Thinking, Design-Activism, Social Innovation and Social Enterprise proved to be less familiar among both Service Providers and Users. Data from the opentext box, requesting interpretations of the terms, mimics the Likert-type scale. In most cases, participants rarely left the open-text boxes answered, only a few did, thus the researcher determined to limit the use or incorporate any interpretation within this section. However, aspects from the open-text boxes submissions will be used later for discussion. Overall, the using an open-text box may not been the most effective tool, and 
perhaps would have been of more value to activity ask these questions within the semistructured interviews.

\subsection{Card-Sorting and Semi-Structured Interviews}

For this segment, a total of 14 participants were engaged. The participants could be divided into further sub-groups: Service Providers and Service Users, the groups were evenly split as seven Service Providers and seven Service Users. Unlike the other two methods within this study, data regarding: age, sexual orientation, or gender was not actively collected, however in some sessions, this information was provided willingly by the participants. This method focused on categorization and thinking process of the provided prompts and stimuli (See Appendix G). Length of the interviews never lasted more than 25 minutes, with the shortest session being four minutes. Interviews with Service Users tended to be shorter, the researcher had made an active effort to not inquire beyond the comfort of the Service User's willingness to share, while also reminding each participant that there are under no obligation to share any information which they wish not to. Overall, Service Users within this segment were observed to express more discomfort and emotional distress, this was observed through more frequent moments of silence and while discussing the subject matter compared to Service Providers.

This session used (See Appendix B) as the loose guide for the follow-up semistructured interview sessions. After three interviews, the guideline was slightly modified, merging Q5 and Q6 from Appendix B to a more simplified question of "Can you share any final thoughts or concerns, regarding this exercise?" The following sections highlights the final sorting cards from both Service Providers and Users. Further discussion of the analysis be found section to be found in Chapter 5. 


\subsubsection{Final Card-Sort of Service Providers}

SERVICE PROVIDER - FINAL SORT

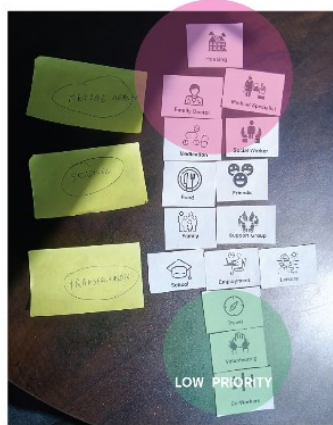

P1 - SERVICE PROVIDER

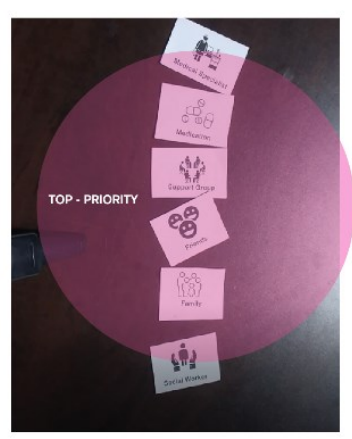

P2- SERVICE PROVIDER
$=$ TOP PRIORITY

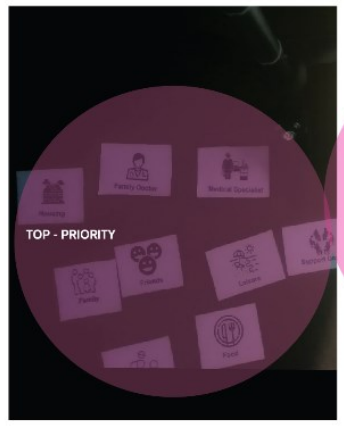

P3- SERVICE PROVIDER
= LOW PRIORITY

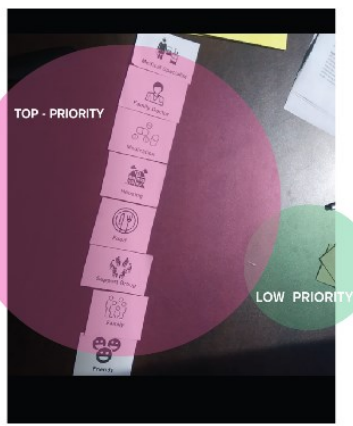

P4- SERVICE PROVIDER

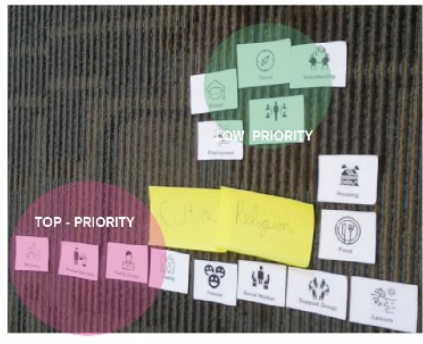

P5 - SERVICE PROVIDER

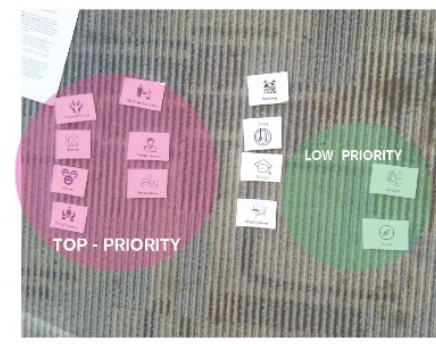

P6 - SERVICE PROVIDER

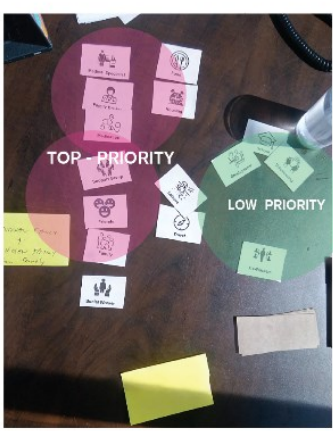

P7 - SERVICE PROVIDER

Figure 2.0 Final Cart-Sorting of Service Providers - High and Low Prioirty

Finding: Two organizing styles emerged amoing the service providers group: Full Sort and Focus Sort. A Full Sort can be described as using more, or next to all of the cards within the deck to organize and compare high and low prioity. A Focus Sort involved not using all the cards, and addressing a particular issues, and in most cases disregarding the low priority cards. Examples of a Full Sort: P1, P5, P6, P7 and Focus Sort: P2, P3 P4

Finding: Service Providers demonstrated higher frequency of asking questions to contextualize the stituation to develop a needs based approach. All particpants shared how their role and job title determined and impacted their decision making. 


\subsubsection{Final Card-Sort of Service User}

SERVICE USER - FINAL SORT

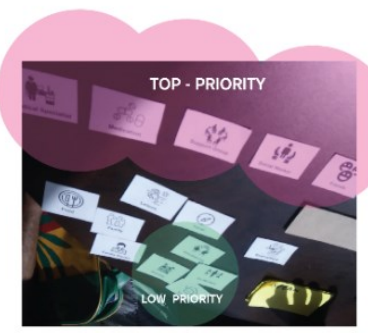

P1 - SERVICE USER
= TOP PRIORITY = LOW PRIORITY

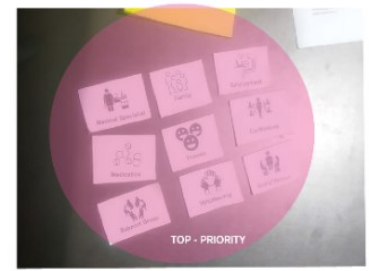

P2 - SERVICE USER

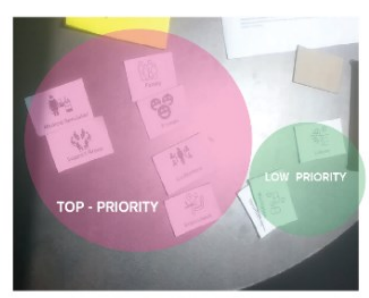

P3- SERVICE USER

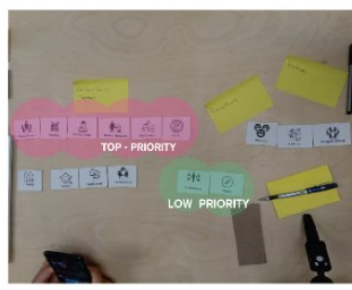

P4- SERVICE USER

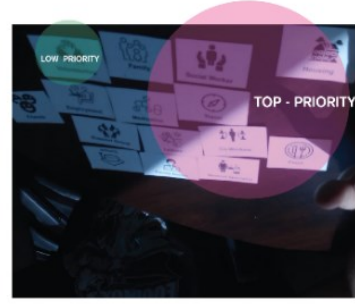

P5- SERVICE USER

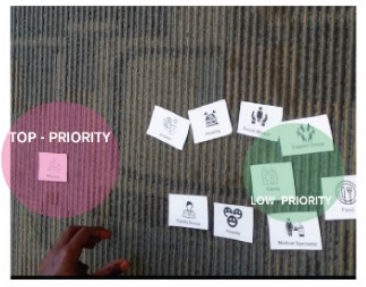

P6- SERVICE USER

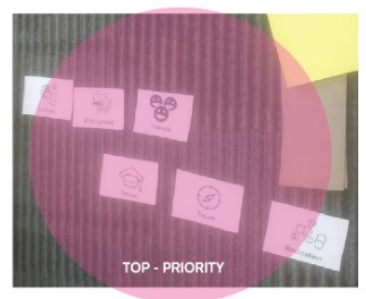

P7- SERVICE USER

Figure 2.1 Final Cart-Sorting of Service Users - High and Low Prioirty

Finding: Service Users had a higher tendency to use "I" statement, often to referring to their past experiences. All participants, within this sub-category shared key moments and memories when recalling their diagnoses. This group demonstrated frequently forms of empathy, compared to Service Providers. Within this activity, the researcher noted it's the ability to "trigger" recollections of almost forgotten memories. P1 shared with the researcher a moments after their diagnoses and how scared they were to walk into a Service Organization out of fears that they would be recognized. Service Users had the tendency to use less cards, only to tell more about the meaning behind the cards their decision-making process, while also having shorter sessions. 


\section{Memos and other observations}

This exercise was intended by the researcher as a means of visualizing the process of decision making while also granting access into the unique experiences of the participants. Service Providers shared that one of their key responsibilities involved enabling Service Users to develop their own the decisions and actions; facilitating the

process with the Service Users' desired outcomes. Frequently, reported by Service Users, were their recollections in regards to the amount of time it took to build trust between them and a Service Providers; it many instances, by meeting others with similar situations which enabled them to gain trust with Service Providers, with contextualize insights from other Service Users.

\subsubsection{Semi-Structured Interviews - SWOT (Analysis) and Overview}

By revisiting the semi-structured interviews, the researcher will admit that this segment resulted in missed opportunities. The interviews became super rigid, strictly following its script (See Appendix B), with an eventual modification, asking participants only for their final thoughts and reflections. In hindsight, the researcher will attest that much of it had to do with feeling vulnerable and incapable after completing such a longawaited ethics application. The researcher asserts that the ethics board may have actively reinforced false assumptions of vulnerability, instilling doubts of the researcher's arsenal of research and community facilitation skills. The researcher would argue that the logics of the ethics board may have in fact debilitated the researcher's confidence momentarily. At the same time, in the spirit of resistance, the interviews have been revisited and outsourced to the logics of a SWOT analysis. Traditionally, a SWOT analysis is used for product development as a means of developing future marketing direction. However, in 
this case, the researcher has subverted its purpose as of categorizing scheme within the semi-structured interviews. In a way, the researcher has "hacked" and subverted its purpose away from a marketing evaluation tool. Followed by the SWOT table are thematic observations which are meant to help contextualize the SWOT table.

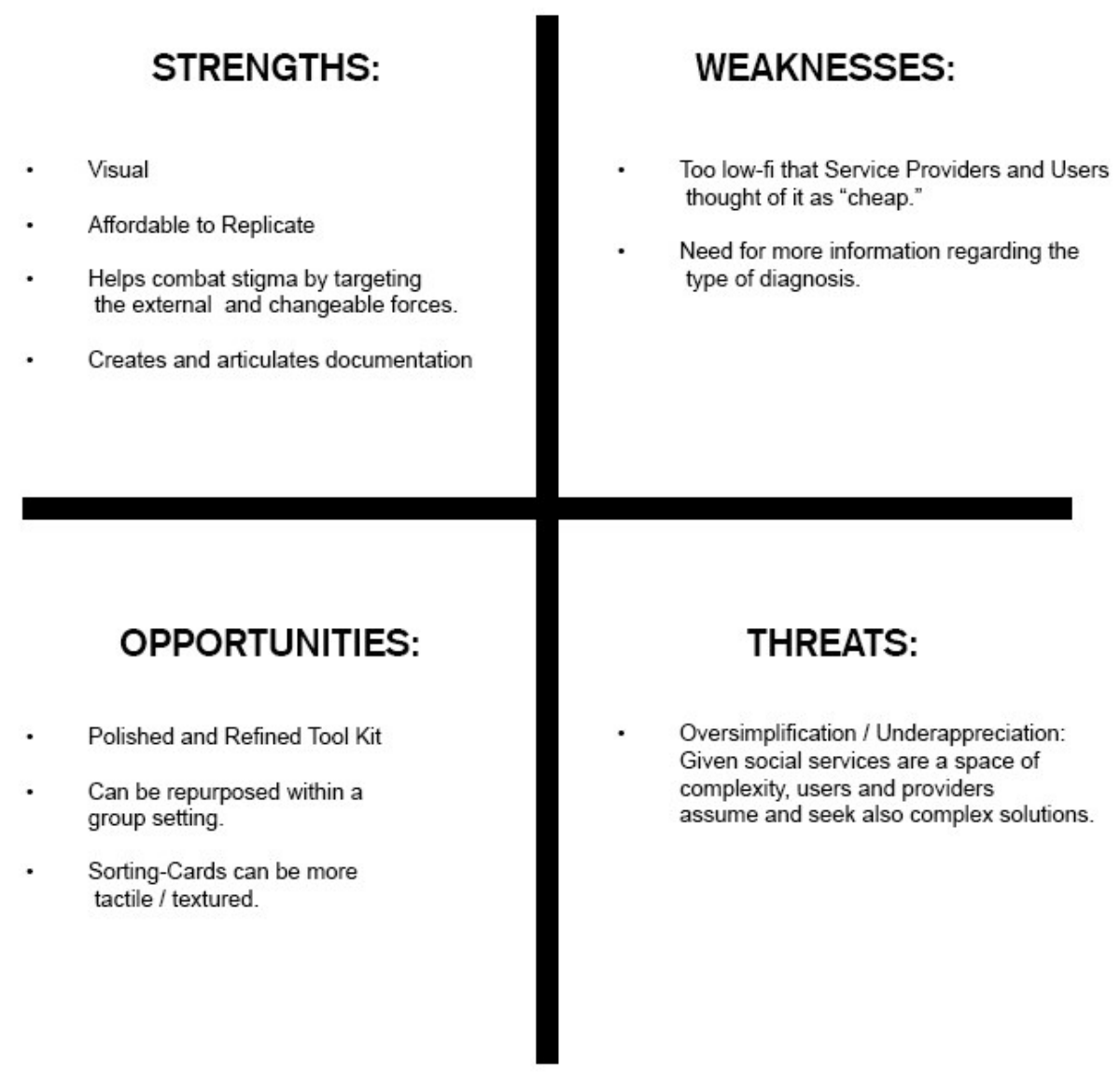

Table 4. - SWOT Analysis of Semi-Structured Interviews

\section{Strengthens}

From the card-sorting, it can be argued that there are more positive gains than there are negative downsides. The visual aspect of the exercise has demonstrated high frequency in it strengthen, as it can help articulate internal thoughts into the external world. Participants have notes that there are challenges when determining desired outcomes, whether it is for health for social development. Its low-fi production fall in line 
with the prototyping methods commonly practiced in design, thus making this activity financially accessible to replicate, however, traditional social work is associated with "conversations" as means of facilitation. In this method, the sorting cards are an aide to facilitating conversations, reducing pressure, potentially on the service users.

\section{Weakness}

Given that disability is such an individualized experience, to assume, that all disabled people, specifically those living with episodic and indivisible disabilities will experience their disabilities in the same fashion as other people is misleading. Further, the context of the exercise forces on the post-diagnosis of a condition, meaning, it fails to encapsulate the whole experience of disability, and inherently starts its understanding of disability from a medical departure point. Often, service providers, within this exercise wanted to know specifically what disability they were addressing, limiting themselves from making definitive answers.

\section{Opportunities}

In response to the weakness and threats, a more formalized "Kit" or "Tool" can be developed from this exercise. Considering that this exercise operated in a one on one context between the researcher and the participants, a group setting would be advising as it could utilize or benefits from a larger focus group. Additionally, considering the development of a formalized "Kit" or "Tool" may elevate the perceived value of card sorting as a research method. However, it does run the risk of reinforcing design as a prescriptive process. Other considerations from the interviews explored the consideration of more tactile artifacts, such as exploring various material consideration for the sorting cards, such as plastic vs paper cards, and even brail of textured groves. 


\section{Threats}

The oversimplification and under appreciating of the card sorting activity may have come from the physical presentation of the sorting cards. The main material used was cardstock and paper. From the researcher's observations, the threats section shares a lot in common with the weakness of this method. Given the multiple stakeholders engagement, this method may fail to be adopted by service providers given their need to validate and report to their funders.

\section{Flowchart and Decisions: Service Provider Vs. Service User}

While influencing factors may vary between service providers, when comparing the decision flow, between adding, removing and usage of the cards, the results are almost next to identical. Ultimately, both groups work towards the same goal of optimal health, yet decisions are expressed through different paths. Below follows a comparative flow of cards, highlighting if all cards were used, added, or removed. 


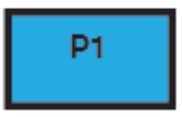

SOCIAL WOREKER (HOUSING)

WAS ALL CAROS USED?

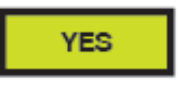

ANY CONCEPTS ADOED?

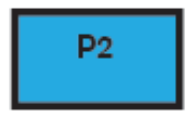

SOCIAL WORKER INCOME
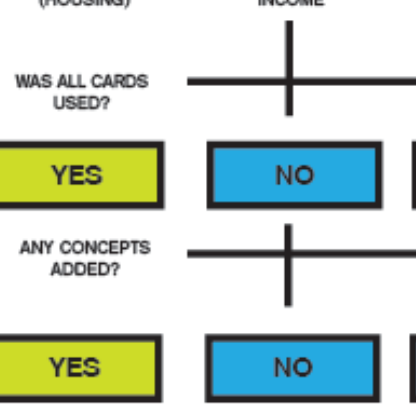

ANY CONCEPTS REMOVED?
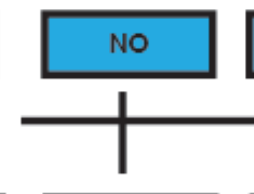

NO

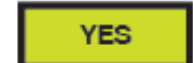

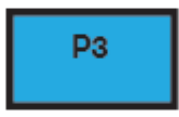

SOCIAL WORKER ETHNO/CULTURE
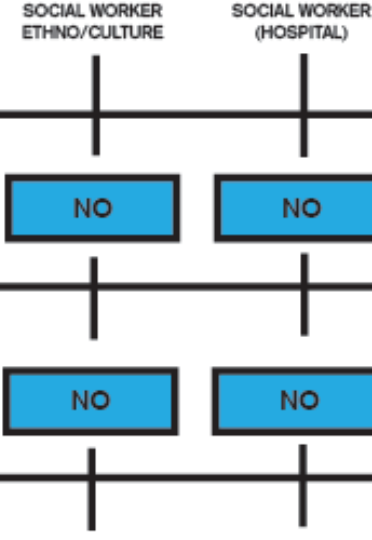

YES

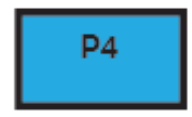

SOCIAL WOREKER (HOSPITAL)
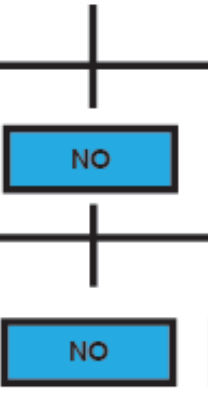

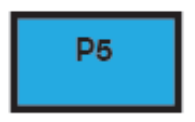

COMMUUNITY OUTREACH (EDUCATION)
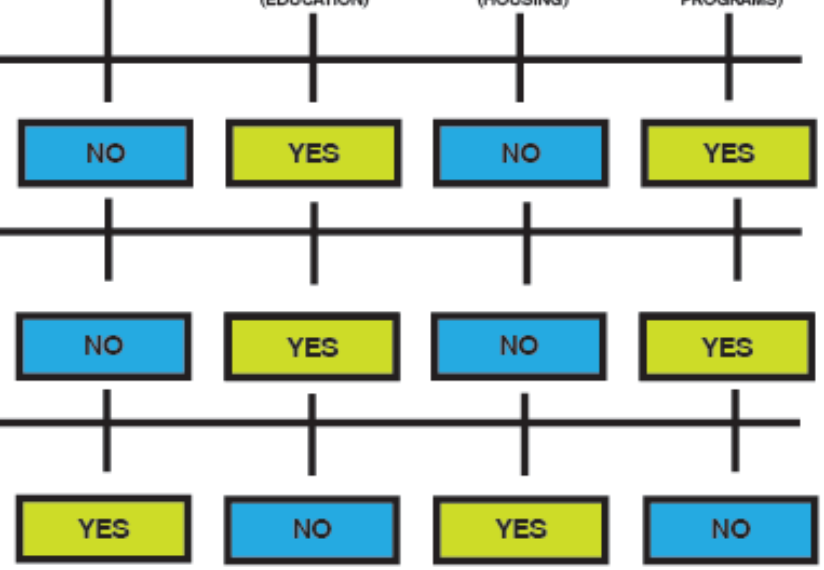

\section{SERVICE USERS}

CARD-SORTING AND SEMI-STRUCTRED INTERVIEWS
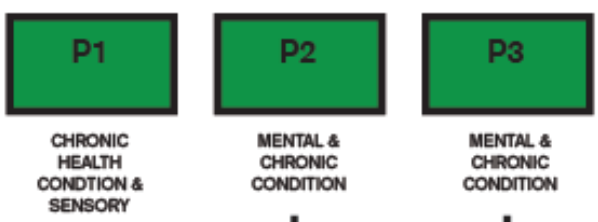

CHRONIC

MENTAL \&

CHRONIC
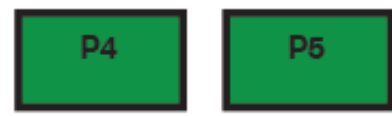

LEAPAING \& HEARENG \&

CHRONIC

CHRONIC
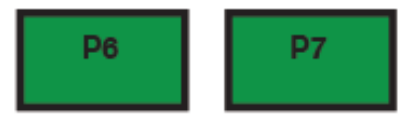

MENTAL \&

CHRONIC

CHRONIC

SENSORY

WAS ALI CAROS

I

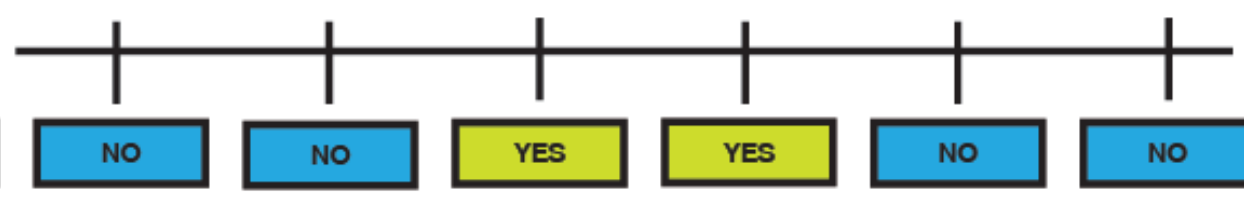

YES

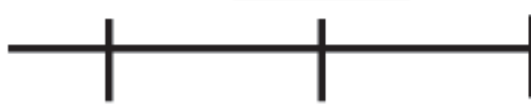

I
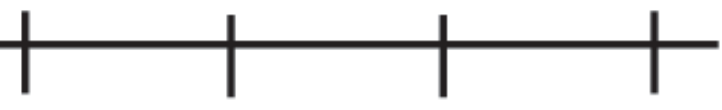

No
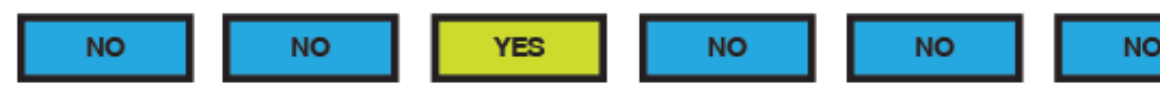

ANY CONCEPTS REMOVED?

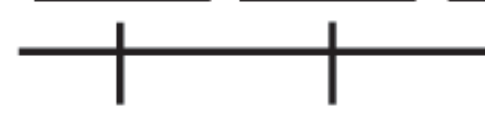

No
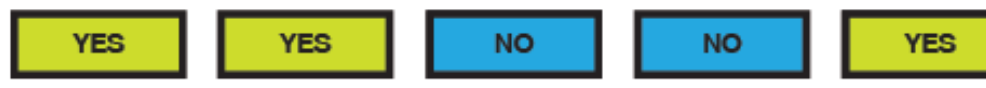

Figure 2.2 Card Sorting Process Flow Chart Comparison 
High and Low Priority - Review of Final Sort Tally

\begin{tabular}{|l|l|l|}
\cline { 2 - 3 } \multicolumn{1}{l|}{} & Service Providers & Service User \\
\hline High Priority: & Medication, Medical & Medical Specialists, \\
& Specialists, Family Doctor, & Medication, Social \\
& Support Group & Worker, Friends \\
\hline Low Priority: & Volunteering, Leisure & Volunteering, Leisure \\
& & \\
\hline
\end{tabular}

Table 5 - Low and High Priority $x$ Service Provider and Service User

The above table is a review of the final sort between Service Providers and Service Users. This table was created through a tallying of the final sorted cards with additional context from the semi-structured interviews. Concept cards that were deemed Low Priority, were identical between Service Users and Providers. A difference between High Priority cards between Service Users and Providers was the presence of Friends and Social Workers among Service Users, while Service Providers championed Family Doctor and Support Group.

Infering from this differences is the importance of choice, agency and independence. Friendships and Social Workers may have scored higher in priority, due to many Service Users' negative experiences attributed to Family Doctors and their limited knowledge regarding a newly diagnosis , or acute medical conditions, thus relying on referrals to Medical Specialists. Among the discussions and interviews with Service Users, Medical Specialists tend to ignore the social aspects of their conditions, such as 
stigma and the disclosure of their access needs. One partiticapnts, Service User P2, articulated their concerns, regarding this partciular situations by stating: "Medical Specialists are simply fancy drug administrators, they are there only to determine and moniter if the drugs are working or not. I don't doubt their knowledge, but I don't feel like myself when I speak to them. " Service User - Participant 3 (SU-P3). In many ways, this mimics what is discussed in the literature regarding medical and service providers' culutural incompentence and social location to contributing (Knight et al, 2014) to poor or inadequate care. Additional, the following highlights mapped out thought of the participants when asked their addition thoughts on the activity.

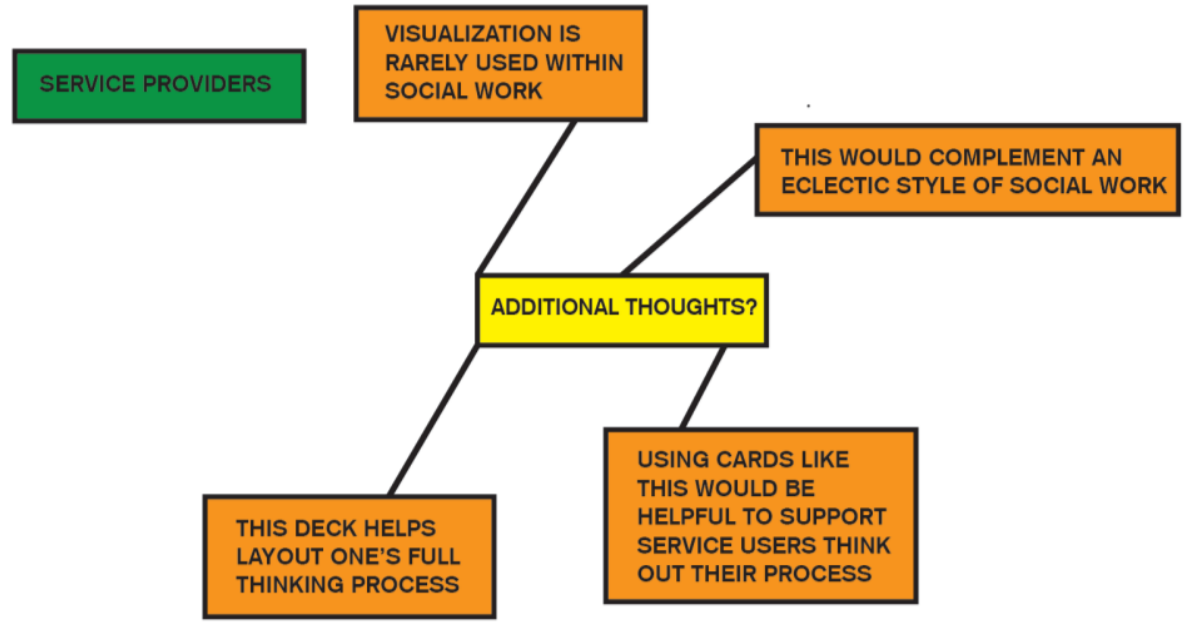

Figure 2.3 - Service Providers Final Thought 


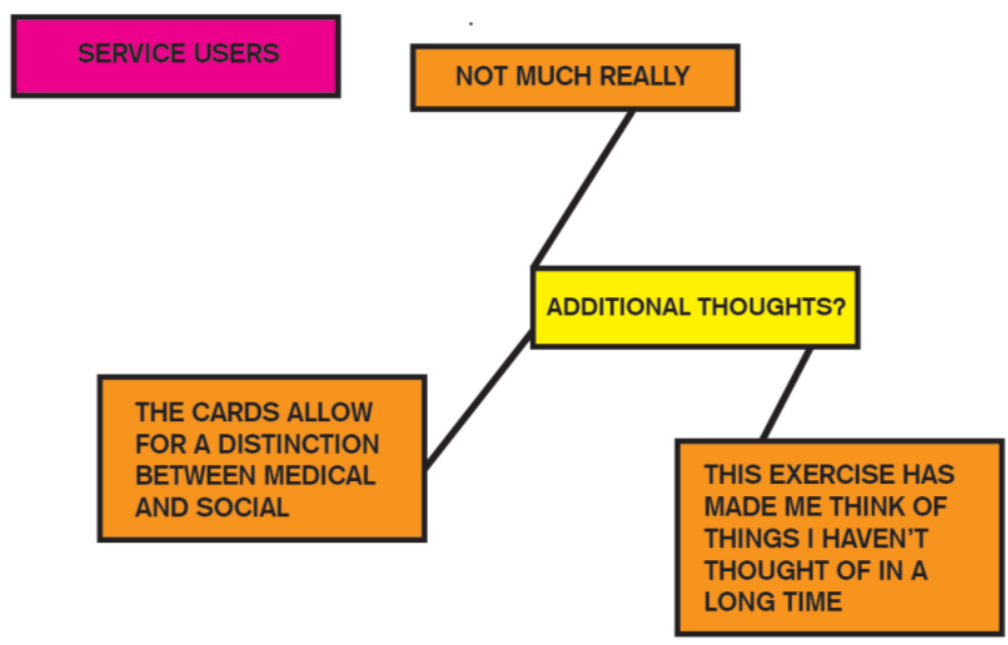

Figure 2.4 Service Users' Final Thought

\subsection{Photo-Voice / Photo Journaling}

The follow sections highlight the contributions and involvement of young queer men, all who access services at LGBTQ affiliated social agencies and are all living with a form of chronic condition or invisible/episodic disability. Their submissions are laid out in photo-essay style and comparative manner, exploring their responses and interpretations to the prompts of: Inclusion and Exclusion. These prompts were chosen considering that critical disability studies seek to challenge the definitions of inclusion and exclusion from the perspectives of disabled people as experts of their own experiences. Further, this method follows the fluid ethos of queer theory (Northridge, Mcgrath, and Quan-Krueger 2007) as the participants demonstrate a disregard static definition. In many ways, this activity evolved closer to align with the more common understanding of co-design and co-creation (Sanders \& Stappers, 2008), as the researcher suggested and embraced recommendations from the participants' request for more 
creative freedom; this includes making original art, particularly within the moment. The images create grant access into their unique perspectives while revealing emotional and politically charged criticism of their environments. Their comments and critiques of their external world demonstrate a shift from understanding disability from a medical to social understanding, which echoes the policy developments in report by Health Canada (2009) on disability and HIV and UN Convention on the Rights of Persons with Disabilities (CRPD).

Participant names, pseudonym names and age are provided. To effectively follow the findings, analysis and discussion, make note of the colour block associated next to each name, to follow and explore the content map shown later in this chapter. With each image, selected text from the participants are shared describing the work, situations and their stories which they wish to share. Warning: subject matter may include explicitly content, such as substance use, social isolation and other "ridiculous queer things." 


\subsubsection{James 24}

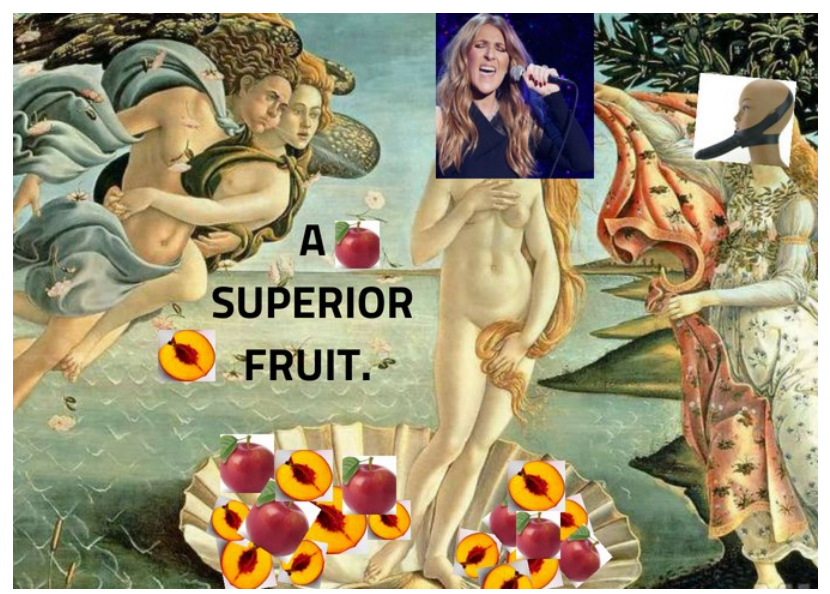

Figure 3.0 Inclusion $x$ James, 24

"It is a really dorky card I made for my friend, that says, on a backdrop of the Venus de Milo, the naked woman standing in a shell. I don't know what it represents, but there are angels standing next to her or something. And then it says, "To A Superior Fruit." And there are really, terribly, cropped peaches and apples in the shell. And on the Venus De Milo, herself is a bad photo of Céline Dion. And one of the angels has a chin dildo attached to it. And what I like about this photo, how it represents inclusion, in a way that it's rare to have queer friends as queer person, that get your particular understanding of queerness, which it's silly and weird. But also, genuine and nice. Cuz it's a card I made for a friend and, but it's got the stuff that he likes and the stuff that he likes is really weird. And it's a nice memory in my head. Cuz gay people cannot be friends with each other." 


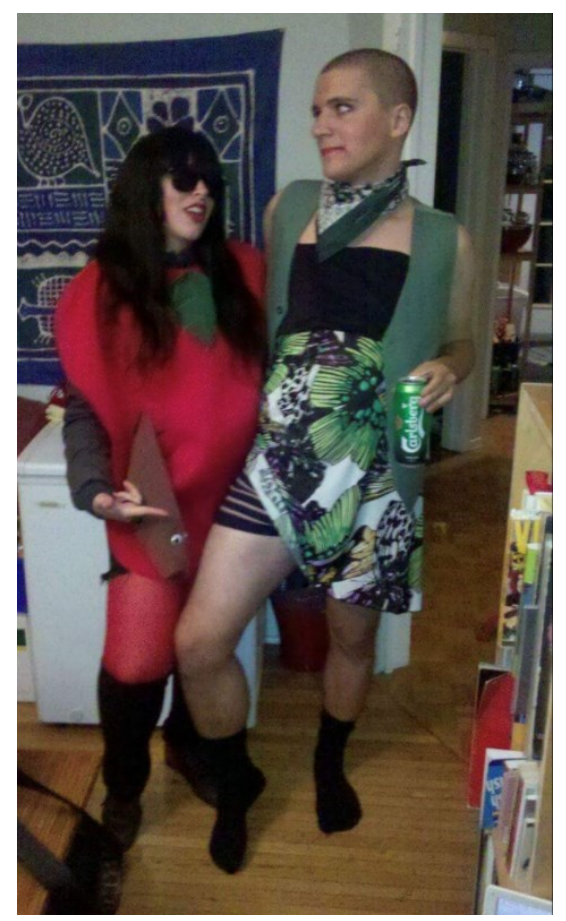

Figure 3.1-Inclusion x James, 24

"That's a seven-year-Halloween in Toronto. I'm wearing the slip of a dress as a top, and a slip of a skirt as a skirt. I shaved my head with a Bic. And I got my friend to do my makeup. We used to go to this punk show as a house activity, a house all my friends lived in. And Halloween was this exciting time, cause everyone was really silly and we're all really poor. I mean, I'm still poor, and it was like representing

friendship, and a time in Toronto, when Toronto was still affordable. That house was right by Trinity Bellwood's, and the whole cost of the apartment was $\$ 1200$ "' 


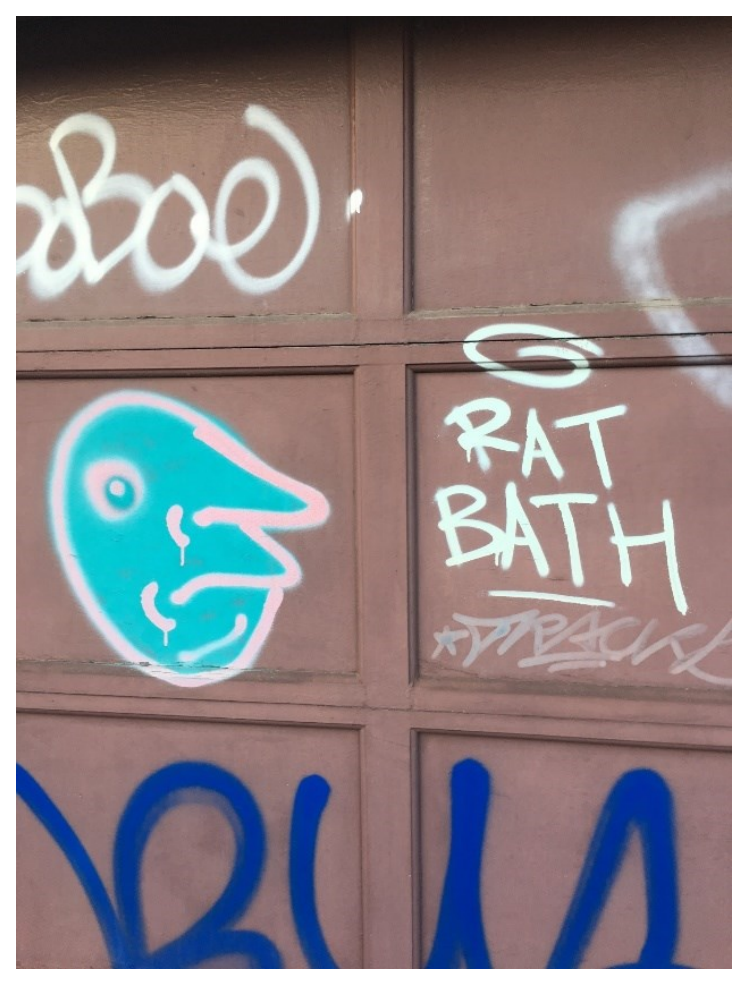

Figure 3.2 - Exclusion x James, 24

"Yea, that is the image of garage Rat Bath, at Ossington and Queen, it used to be a house people would go- where I'd see shows called, Rat House, it's not really about the death of Rat House because it was a disgusting punk House, and no one cares it closed. But the point that is that it represented Toronto as a city of possibility, and I'm from Toronto, thinking of trying to go back, but probably not because it's too expensive. And just thinking about how the places that foster inclusion of a certain kind, can very quickly, thanks to this sort of arbitrary flows of international financialized capital, become completely inaccessible. One of the things we never consider about accessibility is financial accessibility, and the necessity of, like, under gurtting all of our other kind of accessibility with a class analysis. Cause Toronto is like disgusting, like idea of paying the amount of money I need to pay just to work all the time, just to sleep there. sounds like bullshit to me, and I fuckin hate it. But like, I deeply miss it. It's a relationship, like I want to be there, but I can't really be there, and it sucks" 


\subsubsection{Sean, 22}
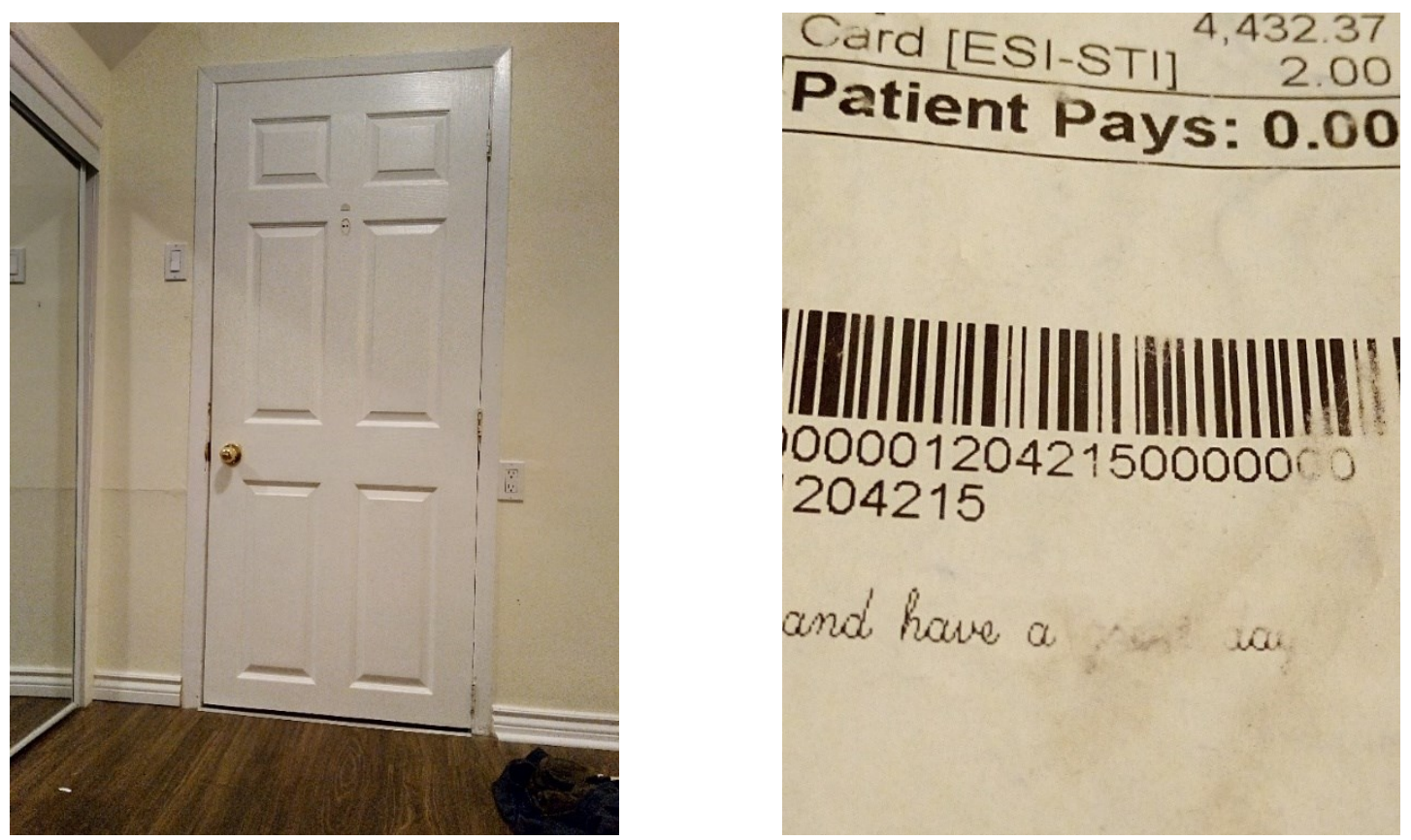

Figure 3.3 on the left - Inclusion $x$ Sean, 22

Figure 3.4 on the right - Exclusion $x$ Sean, 22

"Shared is an image of a door. While some might think it is about exclusion, a door represents inclusion because it reminds people that the capacity to be inclusive involves an invitation to be open. Inclusion requires people to understand the boundaries of space, and where points of access may exist. I choose a door as a reminder that inclusion is a choice and a path to another space.

"This image is from the receipt of my medications. While it may come free, there are hidden costs. I get treated like crap and am constantly told I'm useless because I rely on social supports to pay for these drugs. I may have access to free drugs, but sometimes I don't think it is worth the harassment or shame. Getting help comes with conditions. Sadly, I feel I pay with my dignity." 


\subsubsection{George, 26}
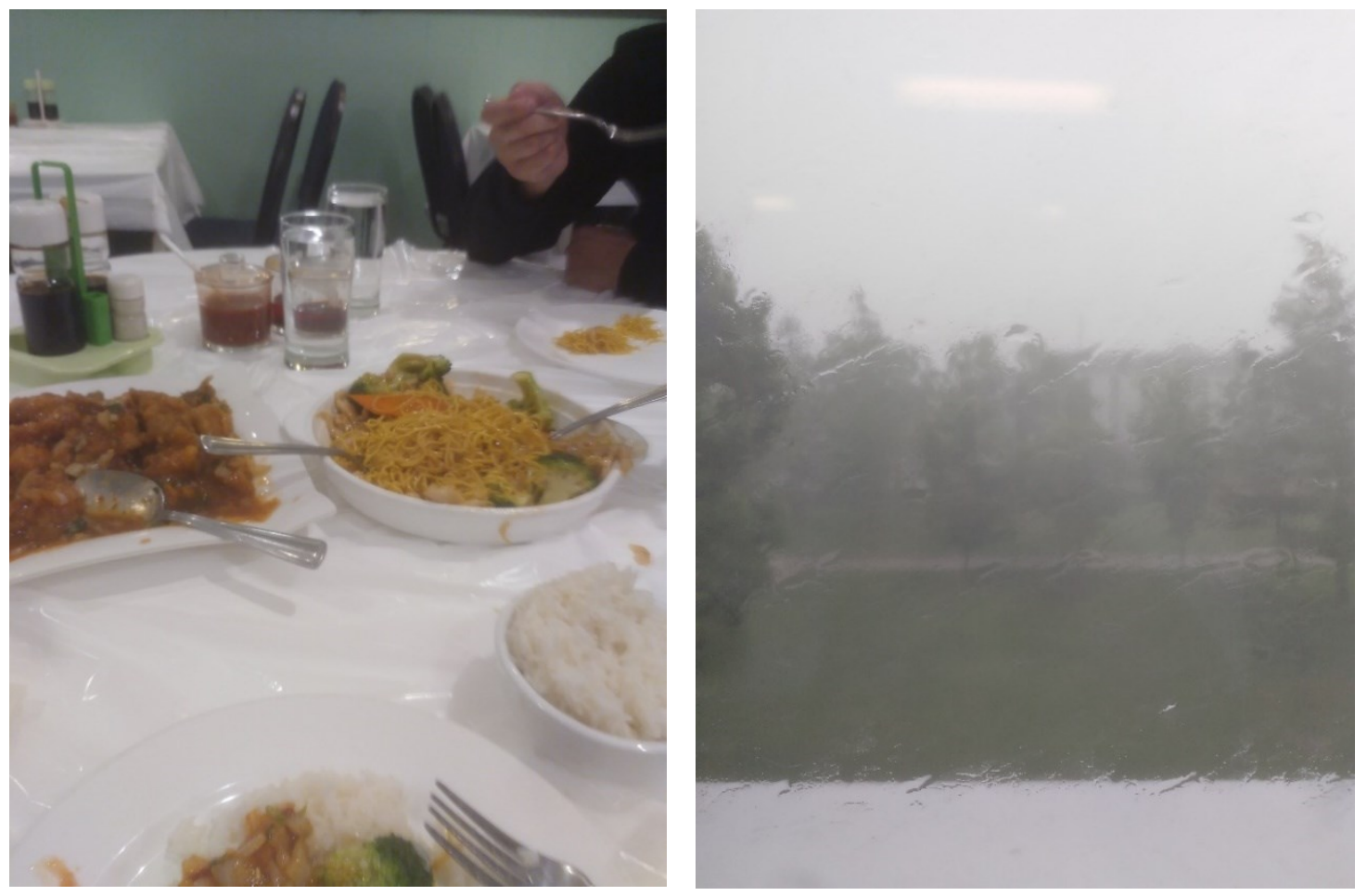

Figure 3.5 on the Left-Inclusion $x$ George, 26

Figure 3.6 on the Right-Exclusion $x$ George, 26

"This is from one of my favorite restaurants. It is a place that is only open in the evening. I feel the food here is so comforting. It makes me feel welcomed on the worst of days. Sometimes I eat here alone, and if I'm ever lucky, I find someone to bring. I feel it take a lot of courage to have a meal with outsiders and weirdos, so anytime anyone wants to eat with me, I feel better about myself. I may not always have the money to eat out at restaurants, but this place feels like it's worth it. They're special times of the day, especially late at night when the prices have a late night special."

"This photo is from outside my window at work. I feel it represents exclusion because I feel trapped at work and must stare into the rain. While the rain may seem violent, inside my workplace is far more hostile than the rain could ever be. In a way, the rain taunts me. Greener pastures are often disgusted, as well as forms of exclusion too." 


\subsubsection{Paul, 28,}

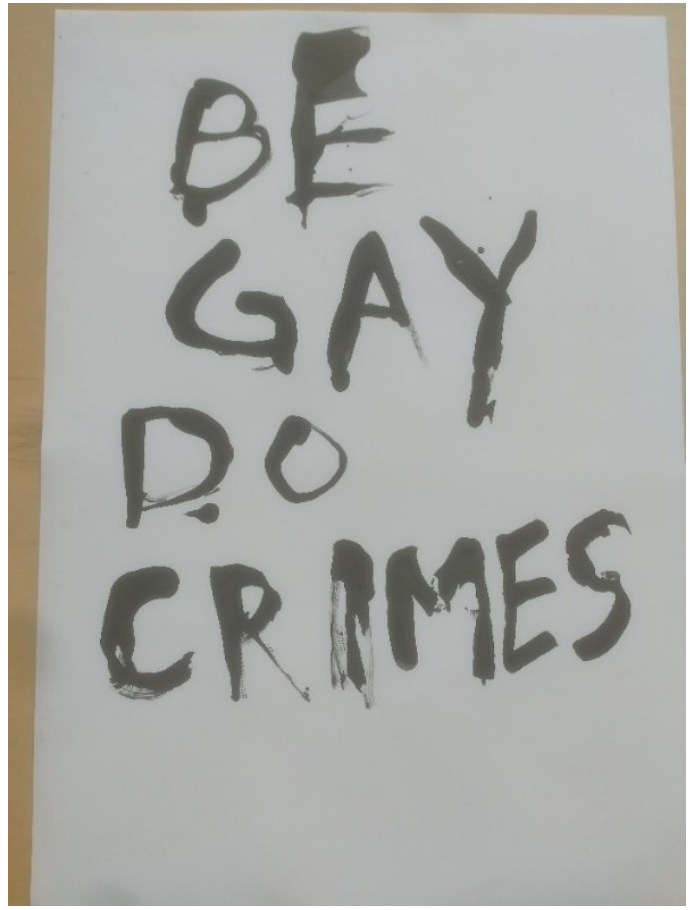

Figure 3.7 - Inclusion x Paul, 28

"The images for this activity were created by me using black ink and a paper straw. While the task asked to take images of the world around me, making and writing as a queer person felt more important. Trying to interpret inclusion for this task seems too abstract, and too difficult to even use just a camera. Words play a big part of being queer and who controls words also controls labels. Some girl I went to college with, who also had the tendency to annoy me often said "Use your words." In many ways, it felt demeaning as if I was too childish to even say what I want. To an extent I think she was right. Finding the words to describe what's going is so far. But usually, and more importantly, when you do find those words, it can be truly liberating. What I wrote and snapped for my interpretation of inclusion are the words "Be Gay Do Crimes." I think I saw this somewhere on twitter and IG. I think it's funny and playful. Some queers are too serious. If you look up the words, I think you'd end up in a deep dive on the internet.

But yea, that's what makes this even more funny. Considering that being gay and queer was once criminal, I think embracing that history and never forgetting is very important. I also think it is important for queers to have access to queer history. And sometimes even searching for forbidden information can be criminal. So, to all my fellow queers, keep searching. And do crimes, fuck with the system, and always riot." 


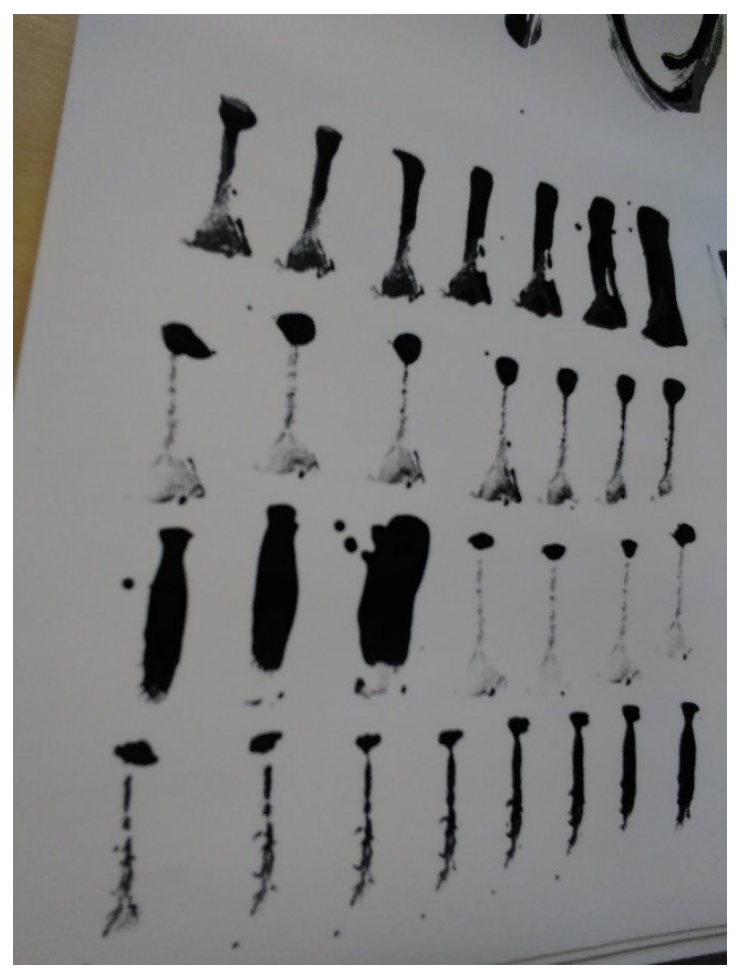

Figure 3.8 -Exclusion $x$ Paul, 28

"I think using this straw and ink technique is a lot harder. Sometimes, I feel by ignoring the instruction I am forcing myself into isolation. I am deliberately leaving myself out. In a way, exclusion has a benefit, but the question now becomes whom may benefit from exclusion? Thinking back on exclusion as a concept, as an expression, I think we often assume as queer people, that it is the majority who operate to exclude us. Yes, I do think, most heterosexual, as most of the world, gain politically from queer exclusion. But somewhere among the mass of heterosexual people, there are individuals who are willing and are truly real allies. Sadly, they too, like us queers, go unnoticed. To effectively articulate exclusion, I didn't use words this time like I made in the other for inclusion. I feel exclusion and inclusion are assumed to be in a binary relationship as if they are often on the extreme ends of each other. Accepting exclusion as an expanded interpretation of inclusion really allows its meaning to sit alone, or at least I think it does. Or maybe the definition is limited to me. Or maybe that's just it, exclusion is about how we limit ourselves and others. Exclusion is a practice of surveillance and policing.

But back to the technique used, it was about expressing repetitive actions, which I feel to be a key component of exclusion. Often the same action will result in different outcomes, but collectively, those outcomes build towards this elusive experience. Exclusion is something felt, and difficult to articulate. In a way, exclusion is about denying and robbing anyone's ability to be themselves" 


\subsubsection{Duke, 24}
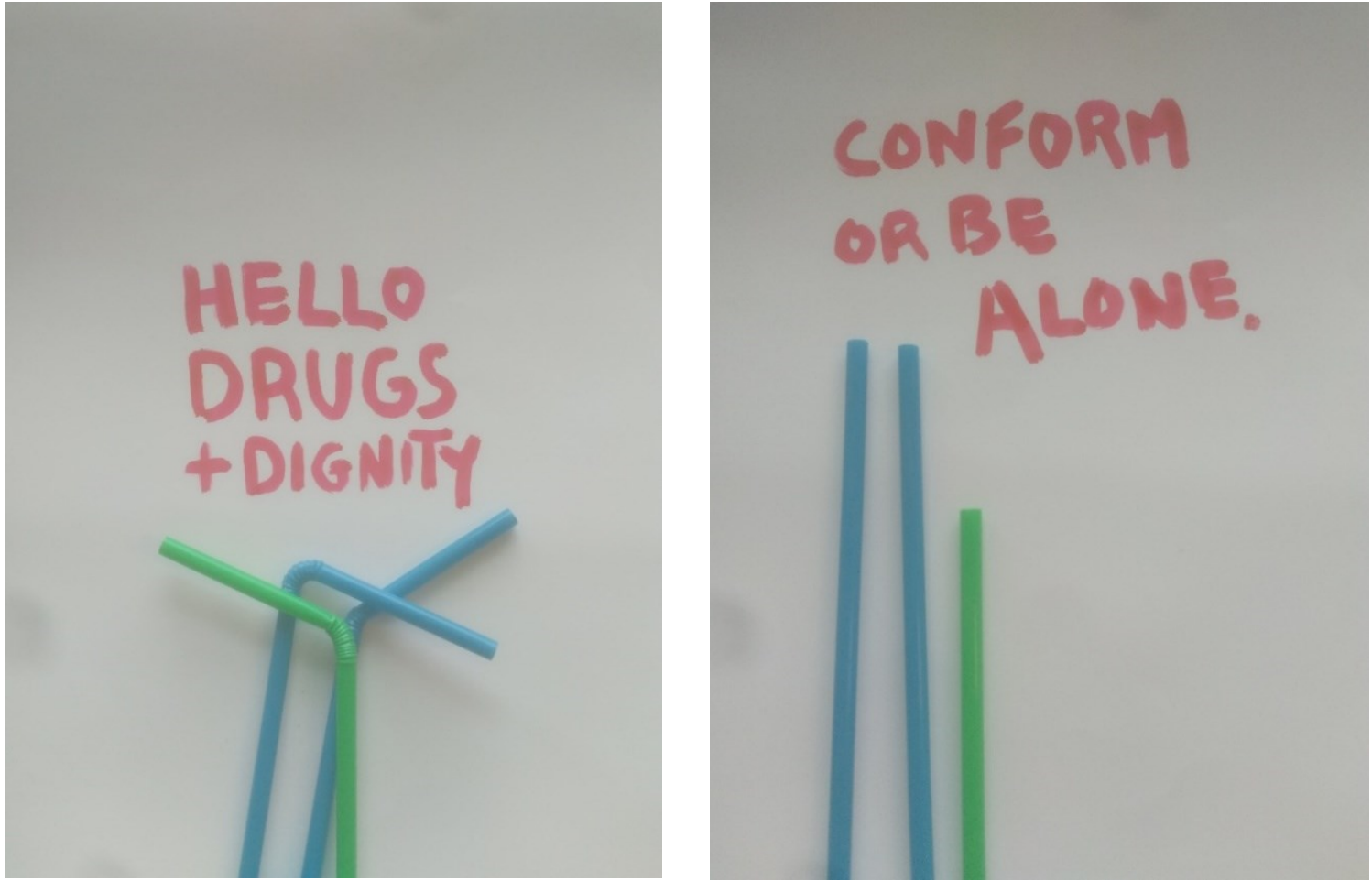

Figure 3.9 on the Left - Inclusion x Duke, 24

Figure 4.0 on the Right-Exclusion $x$ Duke, 24

"Using straws and text, I thought I'd have some fun with this activity. Doesn't seem like research to me, but this activity allows for creativity, and yea, creativity seems to be lacking in most queer related research. So, in the spirit of queerness and saying ridiculous things, I hope these images can articulate inclusion and exclusion. For my submission for inclusion, I've used the words "Hello Drugs + Dignity" I think often take for granted the ability to drink from a cup. To some queers, straws represent a level of freedom, and I often associate freedom with dignity. I've also added the word "Drugs" in there as a reminder of queer substance users. Often drug users are using drugs to escape or cope with difficulties, but in fact what they may be escape are forms of exclusion and oppression. This is why I added the word "Hello" as a friendly greeting

Again, using straws and text, I tried to articulate exclusion as something felt as a result of conformality. I feel exclusion sometimes is deployed as an ultimatum. Strangely, queers are remarkable resilient, however that resilience can be worn down and I think exclusion can do that to queer people. I feel that I tried to articulate this by the words "Conform or be alone" as if our lives are restricted to a binary. Some people may be, others live more freely. The colour code used by the straws is also about race and segregations, also, it's a metaphor for drawing the short straw. In a way, exclusion is process of reminded individuals that everything is their fault." 


\subsubsection{Themes and Motif: Location, Accessibility, Financial and Community.}

In total the four main themes involved were: Accessibility, Community, Financial and Location with a central motif of Self and Agency. The use, and generation of a content mind-map was created from the photos submitted. Each participant was assigned a colour code according to their submissions and placed within the map. It is a mnemonic tool to allow for greater interpretation as an extension of discourse analysis (Witter-Merithew, $\mathrm{n}$. d). The mind map below visualizes the relations and connections between instances. In chapter 5 , section 5.3 will this explore more regarding the themes and motifs.
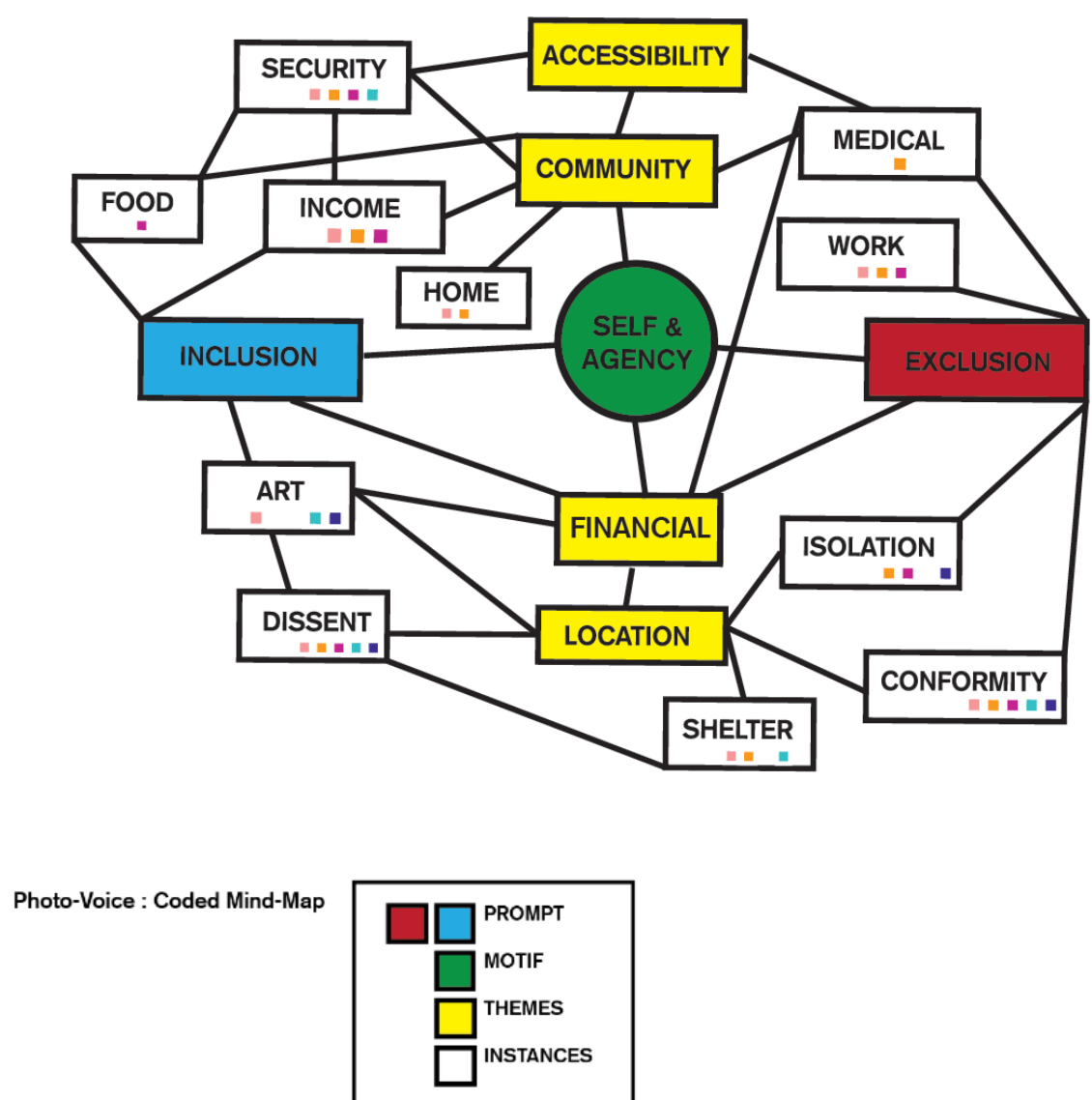

Figure 4.1 Content Map of Photo Voice 


\section{Chapter 5: Discussion}

\subsection{Revisiting the Research Question}

LGBTQ communities and services provided within these communities can be a series of highly complex systems. Navigating and failure to access services may result in additional and unneeded labor, potentially, causing more harm than good. Specifically, this study is focused, and locates its investigation within Ontario, Canada. The current political climate further complicates this study for many reasons such as: changes towards the definition of disability, the reinstated outdated sexual health curriculum and even reduction on social service and health budgets. While this study never seeks to set a new definition on terms such as disability or design, it does seek to understand how these terms are perceived and practiced. As a result, this study provides critical access into potential alternatives and considerations.

In response, this study investigates design research methods in the context of LGBTQ social service agencies and service users, with a focus on Design Activism, Social Innovation and Critical Disability Studies. The motives behind the methods were used to gain a deeper understanding of Design Activism as a practice and how its criteria and consideration may change within the context of LGBTQ communities affected by and living with invisible disabilities and episodic conditions. More precisely, the study sought to explore the following research questions:

Q1: What is Design Activism in the context of LGBTQ communities, living with invisible disabilities and episodic conditions?

SQ1: What is the relationship between Design Activism and Social Innovation? (In the context of LGBTQ Communities living with Invisible and Episodic Disabilities?) 
SQ2: What barriers or limit exists (between LGBTQ service users and providers LGBTQ communities) that result in the discouragement Design-Activism?

SQ3: How might the considerations of Critical Disability Studies and Queerness Theory inform Design Activism as a method / practice?

\subsubsection{Reflections on Ethics Protocols}

The ethics approval process wasn't easy. Especially considering external factors and the researcher experiencing periods of compromised health. The researcher disclosed to the ethics board their disability status and self-identified themselves as a person living with multiple disabilities and as an LGBTQ community member; the self-disclosure was done as a mitigation strategy to inform the board that the researcher commanded a high degree of tacit knowledge when considering the risk and navigation of LGBTQ communities. More importantly, it was done out of respect of the researcher's community and internal social values.

The researcher argued within the ethics protocol that LGBTQ people living with disabilities should not be deemed high risk due to their social location and identities. The response from the board was that they agreed with the false and misconception of marginalization, among LGBTQ people, however still deemed people living with disabilities as highly vulnerable. The ethics board had deemed the methods in review as low risk but required revisions before granting clearance certification. The use of openended questionnaire for self-identifying within the LGBTQ community was requested to be removed and converted towards a closed ended question. Applying the feedback from the ethics board, the researcher did request and validated the reasons to keeping an openended question. The use of an open-ended question was intended to allow participants to self-identify in non-binary responses, as to avoid any imposed limitations on gender and 
sex data.

Consent and withdrawal procedures become more crucial to the point of being overstated. The ethics protocol highlighted that participation within the study was voluntary without compensation. Acquiring consent involved either a digital consent or printed consent form. Two of the three methods, the terms and definition and photovoice, were done digitally and one in person, the card-sorting and semi-structured interview.

\subsubsection{Legitimizing discrimination, but discrimination none the less}

Although not explicitly stated, the researcher would argue that the ethics protocol has demonstrated a minor degree of legitimizing discrimination against LGBTQ people and people living with disabilities, even though it may not have been their intent. This is mainly observed from the researcher's perspectives. Under the disguises of "participant safety," Carleton University's CUREB-B, limited the researcher's efforts to allow participants to self-identify their affiliation with LGBTQ communities. By limiting an open-ended question, allowing it to be feature on method, the terms and definition and not the photo-voice, it further limits the complexity, range and diversity of LGBTQ communities. The ethics concerns default participants into being reminded that they are a risk and danger. Thus, it is the researcher's personal opinion that is the ethics board's operative intent is to mitigate any legal recourse against the school itself, even if it is at the expense of LGBTQ people's right to claim gender and sexual expression. This is how discrimination operates, always thinly veiled, even within an educational environment.

Furthermore, during the review process of the ethics protocol, notable evidence existed proving that the ethics protocol was not fully read in detail. In the original 
submission, the researcher had clarified that no participants' facial features were to be ever captured. This was detailed in multiple appendices. The video recording methods proposed were strictly and solely focused on the artifacts used within the sorting-card methods, with a tilted camera. The only physical featured to be potentially recorded were the hand movements of the participants. From the original feedback and concerns expressed by CUREB-B, it was questioned if faces would be blurred from the video recordings. Given the proposed ethics protocol included "LGBTQ" and "Disability" within the title of ethics protocol, these key terms triggered the ethics board's bias into legitimizing discrimination. After the first round of feedback, the proposed project and methods were deemed low risk, and modifications were applied to obtained clearance.

\subsubsection{Attempting Factor Analysis and a lesson in failure}

Originally, the researcher intended to deploy a Variance Analysis on the selfreported familiarity rankings. Much of the researcher's knowledge regarding Variance Analysis is a direct result of working in marketing related roles and digital advertising (Cost-per-click and Social Media Analytics). To the researcher's surprise, there is a difference between Variation of Analysis, Analysis of Variation, Variance in Discourse, and Variation in Means. This confusion resulted in a hiccup, which can be directly connected to having high hopes and aspirations beyond the scope of this study's capacities. However, it does create a great lesson for discussion and can support the research questions indirectly. Factor Analysis was considered as an alternative. Specifically, Factor Analysis provides to capacity to observe:

"hidden latent variables which cannot be observed directly but are reflected in the answers or variables of the data. It also makes the assumption that there are as many 
factors as there are variables. We then transform our current set of variables into an equal number of variables such that each new variable is a combination of the current ones in some weightage. Hence, we are not essentially adding or removing information in this step but only transforming it." (Perceptive Analytics, 2019).

To extrapolate more meaningful findings from the data, the researcher had bought a pizza for a statistician, Shaundon Holmstrom, from Statistic Canada, bartering lessons and tutorial sessions for the software R (r-project.org), a statistical computing and graphic software. The sought-out support was done, considering the lack of formalized quantitative research methods introduced into the researcher's graduate studies curriculum. Additionally, the cost of any data science training is financially steep, beyond the researcher's access.

Ultimately, inconclusive results were found, and that knowledge regarding Design-Thinking, Design Activism, Social Innovation and Social Enterprise had no bearing on whether the participants identified as a Service User or Service Provider. The following sequences and analytical tools where applied to the data collected in Table 1.

The data from Table 1 was taken from the online form of Terms and Definitions and isolated into a separate excel file, and next uploaded into R. From the uploaded data, specific data packages were use, including "devtool" for making packages run more effectively, "ggfortify" for data visualization, and "psych" package for personality, psychometric, and psychological research, considering the data was measuring familiarity.

Using the 'psych' package, a factor analysis was performed. The first step in this process is parallel analysis, which produces a Scree Plot Graph. (See Figure 1.5 - Scree 
Plot Graph) The Scree plot suggests an adequate number of factors based on "elbows" (i.e. where the slope suddenly flattens) of the graph. The graph produced from the data suggests 2 to 4 factors should suffice. Due to the small sample size, a simpler model with 2 factors was chosen. Between these two factors, at Table 2 - Factor Analysis Results see that Design Thinking, Design Activism, Activism, Social Innovation, are grouped under factor one (MR1). On factor 2, (MR2) Disability related concepts such as Invisible Disability, Episodic Disability, Accessibility, trend together. The underlying response patterns could be grouped under "Design understanding" and "Disability understanding", suggesting two key pillars of understanding for both service providers and users alike. ODSP comprehension does not fit well into either factor, this can be observed in Table 2, as both scores are relatively close from MR1 and MR2, meaning they are .10 of a difference, suggesting understanding of this topic is not well entrenched with the other words in the survey. While two groups may have emerged, the researcher asserts that these groups weak, yielding inconclusive results due to the small sample size.

\section{A Key Lesson and Take Away}

Although the findings yielded inconclusive results, much of the gain from this method revolved around the development of social relations needed to explore quantitative data. Gaining access to training and software tutorials came at the cost of a bartering pizza and a little social grace. The leveraging and building relationships outside of the field of Design can be simple and cost effective. In fact, this form of relationship building, is arguable, a form of disability justice that resonates with Piepzna-Samarasinha (2018) definition. Specifically, the researcher has managed to successfully hack and circumvent a limitation, mainly, the lack of form training on quantitative methods within 
the researcher's educations and financial barrier, by investing in relationships outside of design. For further context and understanding of the oddity of the researcher's relationship to Shaundon Holms, both had met at a video game bar, due the fact that both were new commers to Ottawa seeking new social bonds and friendship. While this method may not directly answer the primary research question, it does highlight the importance of bartering and generative relationships, demonstrating the merging of Crip theory, critical disability and queerness. Calling upon the words of Piepzna-Samarasinha (2018), this segment was about "fucking up, making repair, learnings from mistakes, and showing up for each other" (pg. 127). It also makes a great story.

Admittedly, the technical understanding of factor analysis remains a relative weak point among the researcher's range of methodologies and analysis. However, this attempt was an exploration of the methods' potential to benefit towards the practice of Design. Considering Design as a practice must grow, evolve and expand into new territory (Julier, 2013), as a discipline, would gain by adopting quantitative statistical approaches. This attempt within this study was intended as a mild introduction allowing the researcher to learn more, while also following the experimental ethos of Design Activism. By observing the data, and process from the factor analysis, what can be noted is that factor analysis would operate better with a large sample size. Given the scope, time and resource, this method can be easily reproduced using the exact source code (See Appendix H). Subsequently, the efforts of trying and pursuing factor analysis were more important than the actual analysis itself. A key lesson within this attempt highlights the importance of reaching outside of Design's traditional scope and building new relations. 


\subsubsection{Donut Charts -Affiliation, Most Concerning and Unnoticed}

These questions were intended to help further contextualize this study and potentially capture future directions, as well as understand whom may be contributing within the study. As observed within the Figure 1.3 LGBTQ Community Affiliation of Participants, Gays, or gay men represent a large portion of this study. This is attuned to what has been critiqued on LGBTQ health, as it often remains focused on gay men. (Mulé \& Smith, 2014; Mulé et al., 2009).

When addressing the set of donut charts, regarding most concerning and underrepresentation, comparatively, these questions ask the participants the same question, just in a different tone. Between the two, Racism and Social Oppression both occupy greater concerns within self-reported responses. Strangely, none of the response allude to gay men's health issues at a pressing concern. Specifically, these questions reveal what is already expressed within LGBTQ literature by Roberts and Marx (2018), as LGBTQ people are constantly challenged by means of control, either political or social. Further, these responses can be theorized through Muñoz's (2009) understanding of queerness and evidences, and that it is often difficult to prove these issues, as there are gatekeepers preventing queer knowledge from being understood or validated. This is because queer evidence is "vexed" (pg.65), mainly used to penalized queer people. An example may come from Haddad et al.’s (2017) report on HIV, as gay men, mainly white, represents the largest in population within newly reported HIV incidents and diagnosis. It vexes non-white gay men and anyone outside of this identity, as this report universalizes HIV with all gay men through a quantitative assessment and association (Roberts and Marx, 2018). An emergence of a majority, within an already minority 
group drives and contributes to erasure of anyone outside of the gay-white man identity. Yet, within this study, a gay demographic, although unconfirmable white, whom are suggesting that social oppression and racism are leading issues within the LGBTQ communities, and not exactly HIV or disability. Consequently, it leads to researcher to believer that there is an associated inference, that social oppression and racism are factors of HIV and disability worthy of deeper exploration.

\subsection{Card-Sorting and Semi-Structured Interviews}

From a theoretically stand point, the sorting cards can be argued to be site of queerness, following Muñoz's (2009) definition that queerness is something yet to be imagined; it is a promise of an undeniable uncertainty and potential of a better world; more concretely to be better interpreted as gaze into the horizon, granting one's own escape of the "quagmire of the present" (Muñoz's, 2009; pg. 1) reshaping social relations for the "not yet here" (pg.1.) Both service users and providers are participating in a form of "queer world-making" (Muñoz, 2009, pg. 40), a speculative practice to imagine a better world, a form of utopia, but more contextualized, these participants are imagining the best or better possibility for a person just diagnosed with an invisible and episodic disability, almost and presumable, someone whom is now living with HIV. Their visions are grounded in their own experiences, both lived and professional, while also leaving evidences in the forms of uncharted storytelling, providing a site of evidence ripe for comparing and exploring.

One of the first and immediate findings from this method involved the style of sorting. The two approaches can be best described as a Full Sort and a Focus Sort, mainly from observing service providers. A distinction between the two are is that Full Sorter's 
take a holistic \& interconnective approach, while Focus Sorter's sought immediate problems and implemented remedies. Full Sorters explored multiple issues before finalizing their decisions, while Focus Sorters immediately located barriers and challenges is if they are isolated incidents. The Focus Sorters' approach to disability, is in fact, a target of critiques by Crip Aesthetics, offering a reminder than disability is not something to be fix or remedied (Hamraie, A., \& Fritsch, 2019). While the researcher can assert through observations that none of the service providers intended to hold a demeanor which depreciates their service users' lived experiences, they are perhaps holding barriers and boundaries which protect them from the emotion volatility and harsh realities of the lives of the service users.

\section{Is It Medicine or Social Skill}

From the findings, mainly tally table of High and Low Priority, co-created by participants, as a group demonstrate that Disability mostly remains and continues to be interpreted as a medical concern. This is proven mainly, with Table 5, as an example, demonstrating that a medical specialists and family doctor remain top priority among Service Providers and Service Users. This is further underscored, as concepts assigned to be the lowest of priorities are: Volunteering and Leisure; concepts that would be deemed as social aspects. Admittedly, the context of the question may alter and have direct impact on the results of this study. While the method of card-sorting is meant to make the abstract thinking process tangible and known, the questions and scenario itself, (See Appendix B) is focused at a moment within the continuum disability experiences, mainly, the moment of diagnosed. The aspiration of this study intended to be unique as it captures the moment when one person becomes disabled, particularly due to a medical 
condition. this method ignores and alienates the idea of those whom may have been born with a disability. Originally, the intention of focusing on the post-diagnose experience was influenced by the researcher's own personal experiences health and social navigating systems.

\section{Age-Difference as an influencer}

While not actively collecting age related data, a notable observation made by the researcher indicated that younger participants tended to view the sorting activity from a more holistic perspective, attempting to understanding how concepts interconnect and influence one another. This would be interpreted by the researcher noticing that younger participants had the tendency to review the deck as a whole review, rather than selectively choosing and presenting a final answer. Comparatively, more mature participants focused on instances within their life, limiting the use and excluding more cards from their final sort.

\section{Rare Usage of Post-Its or Additional Supplies}

Overly associated with Design-Thinking are the use of Post-It notes and doodles. While it can be noted that within both groups, the use of any of the additional supplies to articulate their thinking was rare. Potentially, Design-Thinking may need to confront this saturation of "Post-It" notes as the quintessential embodiment of Design-Thinking. While these participants are in fact engaging in a form of Design-Thinking, the researcher asserts that many of the participant are not aware that the card-sorting activity is an extension and a form of Design-Thinking or Design related research methods. Alternatively, the absence or underutilization may be a result of the participants being interviewed in a one-on-one context. With the presence of other participants or peers, co- 
design and co-creation could have effectively improved the facilitation and the use of additional supplies and production of ideas. The researcher had made it clear to all that the additional supplies, pens, Posts-Its, and paper were available to their disposal, yet the additional supplies were rarely used.

On the other hand, this might reflect a larger issue within LGBTQ communities and non-profit culture. Often when given additional resources, there is a failure to value the assets until otherwise proven useful. When comparing the data from the Terms and Definition segment, section 4.1, and the Likert-type scale from Figure 1.3, DesignThinking has shown to be not as prevalent compared to other terms such as Activism. It can be suggested that Design-Think and methods can lend itself more available to the social service sector. This argument is supported further in literature by Nandan et al (2015) citing that social work is not often affiliated social innovation and entrepreneurship, even though social work aims to be a catalyst for social change.

\section{Professional Study Patients and Study Organizations}

Reflecting on the recruitment and articulation this study's purpose seemed almost foreign to many of the participants. Collectively, between both groups, many of the participants have self-reported notable amounts of experience with participating in other community-based research studies, however a majority often dealt with health-related outcomes and its affiliation to funding sources. Sadly, many prospective participants showed disinterest after learning this study was for a school related study, and that the researcher had no financial incentives for its participants. From observations, it appeared that many of the participants are not exposed to explorative approaches with Design, and that financial considerations and compensation played a major role in recruitment. In 
relations to financial compensation, disabled LGBTQ people are correct to reject any participation without financial compensation. In many ways, there are simply protecting their own data.

Bearing in mind, that the researcher has participated in their fair share of LGBTQ related studies and holds an extensive amount Design consideration for surveys; although a potential bias, it was the intent of the researcher to not implicitly lead participants to focus solely of positive aspect of their experiences, to let natural predisposition reveal itself. Concepts such as participant fatigue and professional study patients had occupied the researcher's strategic intent. Potentially this study may find different results should it begin with the intent of measuring or evaluating a programs key performance indicator within an organization. Additionally, these methods can lead themselves even more effectively into a program assessment and expectations management.

\section{Improving the Card-Sorting Activity}

Given the small sample size, this activity may be improved through group participation, or a focus group structure. Through a group-based environment, thus potentially, design can facilitate more co-creation and co-design strategies. Perhaps, this could involve having two or more sets of groups, and have each group design a set of cards from one initial conversation regarding their desired subject. Next steps would involve exchange card decks between groups. From these exchanges, learning about differences between expectations and priorities can be further observed and analyzed.

\subsection{Photo-Voice: Identity and Commanding Narrative}

A total of 7 submissions of this segment were originally captured. Unfortunately, two of those submissions were removed on the grounds that the researcher could 
positively discern that the two submissions in question were not originals or owned by the participant or went drastically against the researcher's instructions for participants inclusion criteria. The data and submissions removed contained images which were watermarked and contained other notable titles of other published works; clearly none of the content submitted was of their own. Furthermore, the description of these images failed to provide any depth or deep considerations, meaning the responses where either a short sentence or just two words. The researcher asserts that these submissions may have been "pity submissions" among the researcher's personal social network. Rejecting these submissions are aligned with Crip theory and critical disability studies, as a departure of disability as a means of pity or charity.

In total, five participants contributed towards the photo-voice segment. All whom identify as gay or queer men. No additional requests were made to discuss their specific conditions; however, the study did require participants to self-identify as service users who live with an episodic disability or invisible disability. Although never directly asked for their sexual and gender information, all participants had willingly disclosed and shared their orientation and gender expressions. Among this sample, all identified as men or male, Gay or Queer and cis-gendered. Among the five participants, no participant was over the age of 30 years. Contrary to this situation, the two participants removed were the ages of 34 and 54 years. Considering the research study was intended to be inclusive of the LGBTQ+ spectrum, the L, B and T of the community fail to occupy large representation within this study. This exact situation is articulated within the literature regarding most LGBTQ projects, consequently, focusing on gay men. (McRuer, 2005; Mulé \& Smith, 2014). While this is not intended to be interpreted as a failure on the 
researcher's contribution, but this in fact does serve as a reminder of how expansive LGBTQ communities can be (Meyer, 2016). Alternatively, it also demonstrates or reflects the limited range of the researcher's personal network. After all the researcher, the research identifies as a queer male.

The lack of financial compensation can potentially be attributed to the low number of submissions. Considering most professional research done within the LGBTQ communities receive funding from major granting agencies, prospective participants can regularly expect and be appropriately financially compensated for their contributions. This insight mainly comes from the researcher's own experiences as a professional research candidate with major medical institutions.

More importantly, considering that the Photo-Voice activity does involve the use of images, a latent drawback is an underlying assumption that the submissions must be "creative" in some artistic manner. Further drawbacks potentially include concerns of limited time as this activity does require a commitment to self-reflection. While no instructions state the need or criteria for creativity, the concept of using images to gain insight into the lives of young men can be tainted and besieged with today's Instagramera and social media expectations of curated content; the lives and identities of these young queer-gay men is given a layer of pressure that may not be present with previous generations. While the pressures of a hyper image-centric culture are pervasive among today's digital savvy generation, participation in such technological focused culture does come at other costs, such as the cost of owning (a high-end) smartphone of device and the attributing brand-identity to self-worth. This instance can be referred in the literature (Brotman, et al, 2015) about older generations experiencing of higher levels of repressive 
politics, mainly homophobia. However, this study can point to potentially towards understanding, and arguing, that the need, or pressure to participate within today's digital savvy arena and social media landscape can be associated with as a new form of oppression. Ownership and access into participation among social media networks do come at the cost of relinquishing privacy and personal data. Negotiating these tension and requirement of consent and privacy, among an emergence of digital technological landscape goes vastly beyond the scope of this study yet provides a critical future direction.

Among the five participants, three individuals had requested to meet with the researcher to contribute towards the study through an in-person session. The original instrument for this segment was devised to be done online solely as a means of mitigating risk. Participants were given instruction on an online form to upload a maximum of five images and a minimum of one, which followed by an open text box. Within the open text box, contributors had the opportunity to express and share the meaning behind the images submitted. Participants within the in-person session followed the online form as a guideline for their sessions.

The in-person session provided an alternative depth into the lives the participants. While the original instructions requested participants to take original photos of their environments, all three had submitted images that they created, two of the participants created on the spot, while another provided an image created from an existing image. When the researcher questioned the participant's submission, and if it fit the criteria for submission, the participant simply took his own mobile device, re-captured his own preexisting work for submission thus creating a new layer of meaning and while still 
following the instructed guidelines of using their smart phone device. As much as the researcher tried to enforce the research guidelines and criteria, this young queer, cleverly, found a way to circumvent the submission criteria. This process of evasion sought a solution that highlights the critical capacities unique to young disabled queer people. In many ways, this act of creativity demonstrates that Crip and disabled queers are possessed with an unusual passion.

Distinctive within the in-person session were the two participants who created live within their sessions. Particularly with the limited materials within the room without any prior knowledge of what materials were available. Images from section 4.3 .4 and 4.3.5, Paul and Duke, are the participants whom executed this activity with such flamboyance and raw emotion. Their materials were simple and commanded a high-level thinking so critical of their environments, their circumstances and while critiquing society at large. The mixed-medium hybrid of visual articulacy, mixing symbols and text, pierced beyond what a photo alone can expectedly do. In a way, the activity demonstrated the importance of giving creative freedoms, especially to young people, and that rigid creative guidelines may result in limiting design activism. These participants demonstrate the importance of mutual respect between researchers and participants; these images articulate with a high degree of potency that strike a nerve almost reminiscent of early AIDS activism; questioning access and power dynamics while also questioning themselves and their community. These instances also demonstrate theoretical alignment between Co-design and Crip theory, as a collision of "world remaking" (Hamraie and Fritsch, 2019; pg. 5) but also as agents of said remaking, allowing for the material conditions to go beyond existence and survival, but the desired outcome of thriving. 
Apart from the three in-person session, the two participants who had utilized the online form to provide incredible insight through images; both whom submitted via mobile captures. Within their images, featured in section 4.3.2 and 4.3.3, Sean, and George, demonstrate self-awareness, like their participant peers. It would be easy to accept that such images, are only mundane objects. In fact, these mundane objects align with what is expressed within the literature by Hackney (2016) regarding the use of everyday objects and activities. Like their peers within this study, these other two participants demonstrate the same level of critical thinking of their surroundings, reassigning meaning to everyday objects.

\subsubsection{Location, Accessibility, Financial, Community and Self \& Agency}

\section{Location}

Location as a theme refers not just to physical spaces, but a state of mind in relation to socio-economic factors. Often participants shared that their external environments influenced their personal views of themselves. Such examples include, James's referral to Rat Bath, a community music venue and Sean's reference to his room door. At the same time, the capacity to illuminate their built environments was a form of negotiation between themselves and their desires. Participant's pursuit of social mobility, their intent and capacity to relocate into preferable outcomes was a constant question, presenting itself in different forms daily as reported by participants.

\section{Accessibility}

Following Fritsch's (2018) definition of access, it can be noted that the articulacy of the participants yields characteristics of both, defensive and offence thrust. Their words, and command of words, demonstrate their conflicts, and their capacity to 
negotiate. Collectively, participants never referred to conditions as means of debilitation, but often citing their disclosure and treat from others as debilitating. Conversations regarding disability and accessibility with their peers often, as reported by participants, often only scratch the surface, solely discussed as "access needs" within their peer networks and academic environments. These instances resonate with PiepznaSamarasinha's (2018) frustration regarding disability and accessibility, which society at large, ignores accessibility and disability knowledge as the pursuit of social justice.

\section{Financial}

This theme goes beyond participant compensation, as all participants, discussed money, power, and privileges in different contexts. Financial literacy has directly impacted each of the participants and their choices, even as far as lifestyles; from where they can live to even costs of medications. Developing financial independence was expressed as an elusive a promise that all the participants could never achieve yet continued to desire. This was more poignant from the words of James, describing the cost of living when stating: "One of the things we never consider about accessibility is financial accessibility, and the necessity of, like, under gurtting all of our other kind of accessibility with a class analysis. Cause Toronto is like disgusting, like idea of paying the amount of money I need to pay just to work all the time, just to sleep there." The cost associated with independence was interpreted frequently as a taunt or mean joke happening at their expense, and out of reach on their own terms.

\section{Community}

Although loosely defined differently between each participant, exclusion and inclusion were deemed directly caused by their communities. As a result, feelings of 
rejections and acts of rejection constituted as form of undesired labour. In order to avoid negative feelings, participants often shared their strategies to reframe their circumstances to feel empowered, reminding themselves that that can change their unhappiness.

Through leaving one's own community, as shared by participants, is what will allow one to understanding the meaning of community. These instances align with the researcher's personal experiences, as they are often seeking or aiming to building community as a response to the lack of community surrounding them.

\section{Self and Agency}

As a motif, there is a constant resurgence of Self \& Agency within this activity and sessions. Within each theme, their actions and comments are directly affiliated with their intent to engage the world. Rather than waiting for permission to assign meaning, each participant reframes, rethinks, and renders a new idea of how they view themselves. Observed within the in-person sessions, along with the text-based submissions, Self and Agency becomes a motif because it is the starting point, direct connection and leading path to how these participants make any sense of their surroundings. This is further underscored by the Photo-Voice process itself, as the participants demonstrate command to invite the researcher into familiar situation, accounting for both implicit and explicit factors and consideration. The participants adopt the importance of calculating their environments and how it may impact them in a range of dimensions, such as mental, emotional, and physical burden. Self and Agency are important because it is central to their negation process. Alternatively, Self and Agency can also be inferred as Authorship and Authority. The participants are publishing their own narratives, telling stories that may go against the assumptions to what they feel are placed upon them. The role of the 
researcher within these situations and context is to be co-designer and [modest] witness (Hamraie and Fritsch, 2019, pg. 5) of the lives of their peers.

\subsection{SQ1: What is the relationship between Design Activism and Social Innovation? (In the context of LGBTQ Communities living with, affected by Invisible and Episodic Disabilities?)}

Liedtka, Salzman, \& Azer (2017; pg. 7) highlight a shift in definition of innovation, with its criteria being a shift in people and process. The first set of innovation, also known as Innovation I, is focused on trained expert designers, and a shift to Innovation II, due to cultivating variance of those who participate in design (pg.13), meaning, who are designers, is brought into question, and the diversification of methods and what is being created and solved are now changed and continue to change. When applying and comparing the definitions of Innovation I and II to Hamraie and Fritsch's (2019) Crip Technoscience Manifesto, there is tension, as their critique, seems to solely focus on Innovation I by critiquing other forms of innovation when stating: "disability is cast as an object of innovation discourse, rather than as a driver of technological change." (pg. 4) While Hamraie and Fritsch (2019) argue that disabled people are experts and designers, perhaps where their argument is misleading is that, both argue for innovation as desirable, and that, for disability to be desirable, it must be, too, innovative itself. From the researcher's perspective, disability is not desirable due to its capacity to innovate, but simply because it is an expression of our humanity. To argue and validate disability's capacity to drive of innovation, is no different than another form of Irving's (2009) "hegemonic bargaining," especially in the form of critical scholarship.

Among Hamraie and Fritsch's (2019) commitments within their manifesto, critical scholarship, practice and activism - critical scholarship remains susceptible as it 
is driven by 'publish or perish' mentality, an unspoken, lurking burden, imbued onto almost every scholar or young critical thinker. For some this pressure can be harmful, for others, maybe motivating. Design Activism researchers, Song and Lou (2016) have also made note of this, reminding that Design Activism needs to move away from the publication-based agenda. For Design Activism to achieve recognition from Social Innovation, practitioners of Design Activism must not be so easily temped by the pressures to publish. In other words, stick to your guns. And learn to love telling stories of your failures. Strangely, this is where Hamraie and Fritsch's (2019) ideals of politicized deign activism realign with Innovation II; it is about messiness and understanding our differences, perhaps even introducing tougher questions such as: How might the Design of a Service embed the need to not exist?

When pointing back to the terms and definition method, it can be immediately discerned and determined that concepts around Design Activism, Social Innovation and Social Enterprise are relatively unknown to the sample of LGBTQ organization and individuals. This is highlighted specially within Figure. 1.2 and 1.3. Comparatively, an argument within social work literature, Nandan et al. (2015) demonstrate the introduction of Social Innovation and Social Entrepreneurship into social work curriculum and cite its importance as a means of responding to the volatile social, economic and political changes within the service landscape. Referencing back to commentary of Seeing Red, Greenspan (2018) critiques the service provider landscape's volatility due to "traditional notions of monitoring and evaluation seldom acknowledge the role of tacit knowledge" (pg. 159). 
To assert a definitive connection between Design Activism and Social Innovation, within the site of this study, seems almost impossible considering, such as Design Activism and Social Innovation remain relatively unknown from the study sample. However, what can be certain is that its relationship is far from static or singular. Further, when compared to the photo-voice activity, its participants share vivid and radical visions to what the relationships are between Inclusion and Exclusion. In some way, they may intersect, lending a narrative direction, like the relationship of Design and Activism, and consequently, as well, between the social and innovation. Perhaps this is because none of them are impaired by their need to be professionals, they simply stay true to themselves, granting opportunities for criticality of their environments and selves. This would lead the research to confidently asserts that: Queerness and Disability is to Activism is what Design-Thinking is to Innovation; they are mutual and interdependent entities allowing each other to thrive and evolve one another. This idea follows Papanek's (1984) "Design is to technology what ecology is to biology" (pg.136), meaning "the mental ability that allows us to change ecosystems (often causing their destructions) also helps us create better systems." (pg. 137); if a system, such as capitalism were built, and does damage, it would be easy to reason that these systems can be dismantled just as they are created, resulting in an emerging new system. Alternatively, a participant did provide their own personal statement, regarding their thoughts on defining social innovation, by sharing: "Honestly, this term to me is synonymous with neoliberalism and I think it should be opposed" - Participants from the Terms and Definition method. This statement gives hope to the researcher as it echoes sentiments and concerns felt by the researcher. Too often has the researcher seen social innovation used to legitimize passive violence, 
discrimination and aggressions against their fellow disabled queer peers, often by queers themselves. Mainly, this is done through language and the control of language; perhaps even more so through the enforcement of service provider and service user identities.

\subsection{SQ2: What barriers or limit exists that result in the discouragement Design- Activism?}

Critical disability studies and queerness provide a stance that help conceptualize barriers to access and inclusion, while also understanding the political charged nature of its surrounds. Referring again to Northridge, McGrath and Quan-Krueger (2007) "We use queer aesthetics to get at truths that are often disgusted by the rhetoric and images deployed by power" (pg. 462). Queerness and Critical Disability Studies emerge the speculative, intangible and technical as a means of allowing LGBTQ people living with invisible and episodic disability to craft and tinker solutions into existence. This is demonstrated by in both photo-voice and the card-sorting activities, both of which the researcher would describe as a form of world-making and remaking (Munoz, 2009; Hamraie and Fritsch, 2019). By attuning to theorical dimensions from Munoz (2009) Hamraie and Fritsch (2019), Northridge, McGrath and Quan-Krueger (2007) and Clare's (2011) coalition cousin-ing of words, Design Activism's generative approach (Thorpe, 2011) can be argued for its capacity to create evidence through means of hybrid storytelling, signifying access to a world that is awaiting to happen. In a way, this may align with Latour \& Woolgar's (1986) understanding of "facts and artifacts" (pg. 176), as a means of creating evidence, meaning, facts and artefacts are constructed, both physically and socially as a means of persuasions rather than objectivity. The artifacts, photo-voice and card-sorting, are created, acknowledging a lack of neutrality. 
Referring to the Card-Sorting activity, one of the weaknesses discovered was the interpretation of Card-Sorting as cheap, and additionally, too over-simplified as a threat. This was noted within the SWOT, Table 4. These claims mainly came from service providers. Additionally, rarely did any of service providers provide any opportunity for self-reflections, an observation made by the researcher, specifically, this was more occurring among the Focus sorters. This has led the researcher to assert that their professionalism, and maybe over confidence in their role as a social worker and service providers limit them from adopting the card-sorting technique as an alternative method for criticality. This can be further contrasted, considering that many of the providers within the terms and definition segment demonstrate minimal knowledge around design and social innovation. Referring to Fry (2009; pg. 248), these service providers are experiencing a form of limit, and that limit exists within their language. When compared to the photo-voice activity, which consist of solely service users, if assumed, like the terms and definitions findings, that these groups do not know much about design activism, the service users, in this activity, disprove the terms and definition findings through their generative artifacts. From the images created, the participants flex and exercise language, which then become an extension of their vocabulary; language, and those who control language can then be argued as a driver and discourager of Design Activism. The subverted use of the mundane, mimic McAlpin's words within Heller's (2013) interview in that "the name of a squad car (Gran Furry) described nicely our anger, and urgency, with humour, a slight camp sensibility, and a nod to the ordinary" (Location, 1974 of 1194). 


\section{Critical Self Awareness \& Reflection as a deterrent/ driver of Design Activism.}

During this study, entertaining grave doubts has been important. As the researcher, one of the major considerations has been around the data, and weather it reveals its meaning, or has it been imposed by my own meanings to fit my own personal agenda? The researcher admits to gaining critical access into organizations where many others fail to gain access, or cooperation, and even at best, a response. Much of this comes from being socially aware, connected due to the researcher's various medical conditions.

The forces that enhance and discourage Design Activism may lay between direct conflict and contradiction of acknowledging one's own social location. If that is so, then deliberating on how activists can actively know themselves becomes critical. A major assumption within this study is that design is what facilitates the connection with selfawareness, whether directly through personal struggle or through a detouring path with varying struggles. Additionally, the researcher is ultra-aware and conscience of the fact that this study is occurring under an Engineering and Design faculty. Historically, the School of Industrial Design has a notable reputation for being technical and skills driven in design. Carleton University and Ottawa are also historic sites with troubled LGBTQ antagonism (Pritchard, 2016). As a result, and direct response, this study is primed, and was strategically chosen to occur within Ottawa and Carleton University for its anticipated hostility and potential reverberating impact. Confronting this history in a productive and meaningful, at times seems too daunting, at the same time, it falls out of the scope of this study, yet it has occupied the researcher's thoughts. Alternatively, rather than seeking what impairs or limits Design Activism, seeking what enables design 
activism may present a more worthy path of subversive outcomes.

\section{Never requested, but freely shared}

Within the process, protocols and interactions, particularly with the service user group, and photo-voice participants, all participants had disclosed their medical conditions. While never intended for collection, what emerged was the prevalence of comorbidity, meaning, participants often had more than one chronic, episodic or invisible condition or disability. Much of this is not a shock to the researcher, however, this disclosure can attest for the additional and compounding stress these individuals experience. Discussed within the card-sorting segment, a few service users reported to the researcher the lack of competencies between their General Practitioner and their medical specialist. Significantly, the researcher would assert that this disclosure highlights are level of earned trust between the researcher and participants. Trusts, becomes critical, because, as the saying goes: Organizations moves and change at the speed of trust.

\subsection{SQ3 How might the considerations of Critical Disability Studies and Queer Theory inform Design Activism as a method / practice?}

This sub question can be distinguished from the primary question, as it more concern more with the technical practice and methods of Design Activism; the primary question is more focused on an interpretation and abstract directives. From the stance of practice and methods, visual elements were heavily deployed within this study. In many ways, the methods utilized reinforce Featherman's (2016) critique of visual reliance, as if visuals and the capacity to "see" is the utmost upper echelon of knowing. Contrary to Featherman's (2016) claim, Buchanan's provides words that ground Design into equitable understanding of design that "there is no reason to believe that parts and wholes 
must be treated in ascending rather than descending order" pg.10. Tiers of knowing are not comparable, however, power imbalances, and various forms of clout co-exist, thus making discernable decisions difficult. Admittedly, the researcher can acknowledge the limited scope of this study as its focus remains on those living with invisible and episodic conditions. However, this study does in fact capture the unseen, as the participants share both emotional impact of their conditions and lived experiences. This is achieved from the photo-voice and sorting-cart activity. Further, the activities, and creation of artifacts are ground the in experiences of disabled LGBTQ people through means of participant and co-creation and co-design (Sanders \& Stappers, 2008).

As a method and practice, critical disability studies and queer theory can breathe subversive air - resuscitating the practice of design which continues to be suffocated by commercial interest. This is particularly achieved through aesthetically means. As mentioned before, the content of the photo-voice can be interpreted as "ridiculously queer", and many ways it aligns with Clare's (2011) comparison of queer and cripple, as a means of forging a politics, and even Hackney's (2016) criteria for mundane reclamation of everyday objects. Clare (2011) and Hackney (2016) demonstrate theoretical alignment, through the lives of the participants. Using the photo-voice activity as an example, many the participants shared mundane and everyday situations as a means of cultivating critique and commentary. Additionally, participants are cultivating more than critique and commentary but the participants are cultivating variance (Liedtka, Salzman \& Azer, 2017) diversifying the narratives and experiences of Disabled LGBT people. A key example from the photo-voice are Sean's images of his medication receipts and Duke's repurposing of straws. Both are simple, yet command an intelligence rooted 
in a self-awareness in subject matters regarding consumption of medications and substance use. Other participants such as James, George, as well as Sean, refer a physical space, either in the form of a room, workplace, restaurant or housing or music venue and assign a social meaning to these spaces. Through their descriptions they are participating "queer world-making” and "remaking” (Muñoz, 2009; Hamraie and Fritsch's (2019). The capacity to assign and reassign additional meaning is done through means of language, and these disabled queer youth are demonstrating what Thorpe (2011) has described regarding designers occupying critical discourse through language. In many ways, these participants are designing their environments and continually critiquing them, while passively critiquing those share occupancies of the similar spaces. Further, participants, both in the sorting card and photo-voice are occupying a speculative space and exploring abstract concepts and translating them into tangle artifacts. In many ways, these activities align with Muñoz's views on queerness as a world yet to be imagined, and only to be further imagined by each participant.

By drawing on the experiences of Clare (2011) and Papanek (1971) and even the researcher's own personal experiences, there is a notable consequence for having views which challenged mainstream attitudes, meaning, ostracization and exile are the immediate rewards for choosing to think differently, or simply existing as different.

Critical Disability Studies and Queerness provide an opportunity to confront own's isolation and the forces that contribute isolation, whether external or internal. Critical Disability and Queerness, or Crip Theory or Crip Aesthetics, or whatever name anyone wishes to call these things, takes a detouring journey into the unforeseen, capturing both spoke and unspoken narratives. Mainly, the detouring is a process or 
hacked direction ensuring the survival of a narrative, otherwise bound towards "erasure" (Hamraie (2016). Ultimately, for queer disabled people to survive and thrive are indicators and presence of "signifiers of accessibility" (Fischer, 1977). The language and practice of Design Activism operates as an accomplice to Crip and Queer aesthetics, this can be achieved by de-contextualizing and recontextualizing the barriers and forms of knowledge that shape identity and artifacts. Again, the photo-voice activity as an example, demonstrate responsiveness and command of words and images, almost like the Fischer's (1977) Gay Semiotics, but rather than focus directly on people, the visual methods focus on the experiences and its capacity to articulated in an alternative semiotic or arguable a form of future making. This is mainly done through language and words which has been supported by Thorpe (2011).

\subsection{Summary: Design Activism as an Interdisciplinary Faction of Design}

Before beginning this study, there was serious debate about developing community partnerships to conduct the study. Included in this discussion involved getting letters of support that would promise resources, and guarantee access to study candidates. The researcher asserts and would argue that the need for community partnerships is almost ritualistic and empty in meaning, like Young's (2014) definition of inspiration porn. From the researcher's own experiences and observations, the need for community partnerships has historically been more indicative of researchers not being part of the communities they are investigating. The use of community partners grant access to protected communities, and disguises that the research may be potentially operating with an alternative agenda. In the case of this study, the researcher can confidently share and identify with the participants being studied, as they too, self-identify as an individual who 
is part of various LGBTQ communities and is living with multiple permanent episodic conditions. Additionally, the researcher has held various service provider roles, thus understanding both service and provider experiences. Rejecting community partnerships and perusing this study independently, while leveraging known contacts within the LGBTQ communities has been part of this process and part of the much needed "friction" (Hamraie and Fritsch, 2019) to expand on the meaning and exploration of Design Activism. Through the combinations of methods: Terms and Definition Review, CardSorting \& Semi-Structured Interviews, and Photo-Voice, all aimed to confront and locate the contradistinctions of LGBTQ disability. Overall, the researcher would selfproclaimed themselves as, distinctively, Professionally Disabled, meaning, they operate somewhere between designing one's "own tools and environments", such as the sorting cards themselves, and "using experiential knowledge to adapt tools for daily use", such given participants more freedoms within the photo-voice activity, as well as "engaging in professional design practices" through means of self-reflection. In other words, it is the clashing, and constant shifting of multiple operating identities, collaborating in tandem that enables Design Activism to be an interdisciplinary in its practice, research methods and framework. Even if its occurrence operates within an internal stage, bound by the confines of mental abstraction. When practice beyond the individual's internal dialogue, perhaps this is when design activism becomes truly more exciting and explosive in potential.

In contrast, there is a strange feeling reflecting on the past, and for foreseeable future. Particularly in relation to the newly developed and continued acquisition of design research and design activism skills gained from this study. As this study ends, the 
researcher feels worlds apart from where they began, and begin to entertain doubts of my own inclusion with various LGBTQ communities; it is both frightening and exciting; however, this might be an indicator of the own's own truth and liberation. Finding pleasure and joy in exile and isolation is too much of a foreign concept that others may fear to entertain, however it seems to be the only way to seek comfort in the face of complexity.

Followed within the next chapter is a somewhat quasi declaration, an almost micro-manifesto, or hollering statements, echo-locating forms of self-reclaiming love. Its aims are to clarify further what design activism may truly be within the context of LGBTQ people living with invisible and episodic conditions. Gained from this study is a deeper understanding of the importance of language and its capacity to borrow and breath subversion into the mundane. Reshaping language, to reshape one's own environments can grant authorship and authority of one's own narrative. While Design-Activism may be a lesser known term among LGBTQ communities within this study, it proves that there remains much more to be explored. Design Activism and Design as a whole. Undoubtably, many future opportunities of exploration at the intersections of Design and Queer-Disability, it remains a matter of inviting and supporting those who may envision those futures. 


\section{Chapter 6: Conclusion: What is Design Activism?}

\subsection{LGBTQ, Disability and Social Innovation and the Micro-Manifesto}

Design Activism is the relationship between Design and Activism; more specifically, Design precedes Activism as an indicator of strategic intent. Design Activism is the conceptualization to which loosely guides action, and in some cases, only to be articulated as Activism. Design Activism is a metaphor and literal narrative, of a healthy, mutual and interconnected planning between two or more entities, both of whom learn and prepare to depart ways. It is the liberation of the two that grant the vision and promises to those who practice Design Activism. Design Activism begins at an abstract level, leaving a trail of its existence in the artifacts that amplify and empower the disregarded. In many ways, Design Activism is multimodal. Design Activism is the anger of many gender diverse bodies, further accented with the lisp of a million more queers. Design Activism is about being justifiably angry, and commanding candor and wit to cut through the bullshit. Design Activism is at first, mocked and ostracized, then aggressively doubted, and lastly, always self-recognized as undeniable. Design Activism mimics a closeted polemist's internal negotiations to receive and provide critique. Design Activism swims against imposition, challenging the enforcement of moral certainty over reason and evidence. Design Activism confronts exile and ostracization as an opportunity for personal development. Design Activism is the courage to love when you are denied your basic human desires. Design Activism is the intent to believe in a better world than the one you have been forced to inherit. Design Activism is looking into a broken mirror, and being shamed into believing everything you see is wrong and worthless; you will be 
tempted to shatter what you see further; when you do, you realize from these shards, and from all the disappearing hate, you can, make a fancy disco ball. Design Activism is the commitment to remain fiercely kind, to yourself and others, when all self-compassion runs dry. Design Activism is the dream to imagine change when one feels hope is no more. Most importantly, those dreams and desires, although they may go unnoticed, the lack of acknowledgement does far from diminish its existence. One's very own existence becomes a site of resistance. As much as Design Activism yields authority from dissent and difference, Design Activism is equally vulnerable to appropriation. Much of Design Activism's articulation resides in the political intent of its practitioners and its capacity to rethink and redirect the current narrative.

Design-Activism has historically always existed within the LGBTQ communities, this is observed from the researcher's own observation and tacit knowledge, and Heller's (2013) Branding AIDS; only with Design operating in a lower frequency, going unnoticed and overlooked. For those whom have always known, this may as well have been their little secret and joy; and as you now read these words, you the reader, are now part of that little secret. Design Activism operates in a humbler manner compared to its independent sibling, Activism. To call this occurrence by name is to highlight one of Design-Activism's intrinsic quality. As a result of this study, a term best suited for Design-Activism's unseen capacities, as contributed and influenced by Disabled LGBTQ people, is a term which the researcher describes as an: Undetectable Work-Ethic.

\section{Undetectable Work-Ethic}

To really understand this term, one must try to imagine the struggles, efforts and relentless dedication of LGBTQ communities who have fought for liberties that many 
LGBTQ communities take for granted today. While many of their struggles have changed, they are not forgotten, they remain, they just happen to be not as prominent. Like the framework of Disability Justice, through "relationship building and fucking up" (Piepzna-Samarasinha ,2018, pg. 127), an Undetectable Work-Ethic is a lexicon of labour, aligned, by borrowing from Fischer's (1977) "lexicon of attract" and Hamraie and Fritsch, (2019) rejection of compliance as a form of labour. This "lexicon of labour" permeates beyond and between boundaries that aims to critique labour itself through generative artifacts and critical word slinging, building towards a collective, messy, experimental, frictional, and generative (Hamraie and Fritsch, 2019) future. It is the borrowing of language that grants its authority in face of contested terrain.

The term Undetectable is borrowed from the scientific language regarding HIV and its latest advances and discoveries. Specifically, the term refers to the viral presence within an HIV-positive person's blood. The viral concentration is so low that traditional tests cannot detect its presence. Additionally, this information was declared within a consensus statement after two studies (Prevention Access Campaign, 2018) have proven that an undetectable status has proven untransmutable through sexual activity and blood to blood contact. While queer critics and theorist such as Warner (2004) have argued that "queer knowledge should not seek the social in the medical," (pg. 335) it is the very act of rejecting and resisting this claim to which queerness truly exists; queer theory and queer people must, at one point, reject its own theories and ideas. Breathing subversion can be achieved by rejecting that medical and social are in opposition and accepting that both are in fact teammates, co-designing and co-creating opportunities for a better outcome. Indirectly, an Undetectable-Work Ethic is combative while also in stealth and 
non-confrontational, while also being confrontation when needed. It is the embracing of this exact conflict that allows new queer and critical disability knowledge to develop. By seeking the social in the medical, and redirecting terms, Critical Disability and Queerness generate new language that circumvent barrier that reinforce the debilitation of disabled queer people. It is through self-awareness and critical reflection, which can be achieved with Design-Activism. Admittedly, it the process of unseen labour, whether emotional or intellectual which demands the praxis of Design-Activism. The practice of Design Activism, as strange and challenging as it may be, is always worth the pursuit, whether for justice or for truth. Design Activism promises a learning journey to destinations where traditional and institutional schooling could never go.

\subsection{Future Recommendations}

From the study, there are many potential recommendations, however, not all have equal explosive potential towards contributing towards Design. The following are the top key recommendations along with a brief insight supporting the future direction.

\section{Recommendation 1: Design Activism in Digital Spaces}

Given that the instruments used within the study, two of the three methods, the terms and definition and photo-voice, mainly relied on digital forms of technology, such as the online forms and participants' smart phone devices. It would be interesting to understand how the adoption of new forms of digital technology are enhancing participation in Design Activism or Activism efforts. This approach seems highly feasible considering the capacities of digital technologies to implement surveillance of subject participants. Alternatively, it could be explored how digital technologies are a deterrent given that all data can so accurately document. 


\section{Recommendation 2: Understanding Race and its relation to Design and Desire}

Considering that the researcher identifies as a visible minority, there were notable hints and forms of hostility towards the researcher when discussing the scope of this study to prospective participants, particularly from service providers. In many ways, it is highly understandable why service providers would be protective of their experiences, specifically considering the confidentiality and sensitivity of the subject matter. However, to continue to believe that race related issues do not have relevance towards Design is a commitment to ignorance. Design is inherently political, as well as race related issues. Adopting a decolonial approach would potentially elevate the future studies within the area of Design Activism. This direction is support by both lived experience of the researcher and literature, but also from the fact that most critics and published commenters within the area of Design Activism are white males. (i.e. Julier, Papanek, Grudin, Fuad-Luke). In contrasts, it is often visible minorities and outsiders, LGBTQ people and Disable people who are practicing Design Activism, as argued by Ahmed, (2018) in higher concentrated efforts with the added burden of the work being done as free labour, yet never receive the critical appraisal or recognition. Comparatively, the findings from Figure 1.7 and 1.8 hint towards race related issue and social and political forces beyond one's control are site worthy of further investigation.

\section{Recommendation 3: Exploring the relationships between Service Providers to Service Provider and or Service Users to Service User (Peer Networks)}

To move away from the binary relations, as discovered within this study, exploring the mutual agreeing or competitive dynamics between service providers might allow for understanding how agencies compromise the services they provide. This can be further contextualized as many agencies are often competing for similar government funding 
streams. Sustainable funding models plague non-profit culture often going overlooked. This can be explained by design's obsession with "users," failing to target those who support the users. Additionally, considering the sample size of this study, an expanding sample would be further beneficial, as well as doing a study between provincial boundaries, as most social and health service fall under provincial jurisdiction. (Greenspan, 2018; Hindmarch, Orsini, Gagnon, 2018).

\subsection{Final Self-Reflections}

There are a lot of failures and missed opportunities within this study. In many cases, the researcher asserts that most were intentional, perhaps. The thought of failure may scare many away. Failure is often deemed as a negative attribute, but gladly, queerness and disability, when considering design, deem it far from true.

As the researcher of this study, exploring one's own responsibilities becomes a hidden theme, and those additional themes never make it to these pages. Emerging from this study addresses the conflicts involved in capturing new knowledge or even validating different ways of knowing. There are many conflicts with being a queer researcher, more particularly, being a queer researcher living with a disability and chronic condition; this plays a heavy burden which some may never know or understand, and that's becomes my secret to keep and cherish. While this burden may be an exercise of social isolation, it becomes an artifact of one's own truth. Design Activism, like all good design, "renders itself invisible while simultaneously magnifying the scope of its user's will" (Grundin, 2010 pg. 30). In other words, Design Activism is about one's own liberation. 


\title{
Appendix A
}

\section{Sort-Card Activity and Semi-Structured Interview Informed Consent Form}

\author{
Title: Design-Research and LGBTQ+ People Study
}

Date of ethics clearance: June $11^{\text {th }}-2019$

Ethics Clearance for the Collection of Data Expires: June 30 2019

I choose to participate in a study on Design-Research

and LGBT+ People. This study aims to understand existing knowledge about Activism, Entrepreneurship and Disability and how it can inform Service Design. The researcher for this study is Juan Saavedra in the School of Industrial Design, in the Faculty of Engineering and Design. He is working under the supervision of Bjarki Hallgrimsson, School of Industrial Design and Dr. Xuan Thuy Nguyen, The Institute of Interdisciplinary Studies, Carleton University.

The purpose of study: To explore considerations and criteria to improve Service Design for social support programing while considering the complexities of LGBTQ+ Sub-Cultures of Activism, Entrepreneurship, and Invisible Disabilities

To be eligible for this study: you must be 18+ years of age; residing within Ontario, Canada; and must self-identify with the LGBTQ+ communities. You must either be living with an invisible disability or volunteer/work in a professional capacity as a service provider for these respective communities. Participation in this study is voluntary.

Task: This segment of the study will take between 20-60 minutes max. With your consent, interviews will be audio-video-recorded, and solely focused on focused on the visual-cards. You are encouraging to think aloud. No facial features will be captured. The focus of the video recording is indented to document the visual cards and the way in which they are organized by the participants. Once the audio recordings been transcribed, the audio-recording will be destroyed, and the images will remain and used within the study along with transcriptions. Your wish to not be audio-video recorded is also an option, simply let the researcher known. No penalties for choosing this option.

This session will ask you to organize a set of cards and articulate your process of organizing and how it is informed by your experiences and knowledge as person who identifies as part of the LGBTQ+ communities. The set of cards have been informed around issues of Activism Entrepreneurship, and Disability. This activity is known as Card-sorting and will be followed by a semi-structured interview.

Compensation: There is no compensation as participation is voluntary. However, there will be light refreshment provided within this session.

Right to Withdraw from study: You have the right to end your participation in the study at any time, for any reason, and without providing reason. You can withdraw from the session and at any given moment, including while participating in a session. If you withdraw from the study after completing the sessions, all information you have provided will be immediately destroyed, however, you must contact the researcher within two weeks to effectively withdraw. All data will be coded and have identifiers removed after two weeks. You have up to a max of two weeks to withdraw your data should you wish. Withdrawing from this study will not impact your access to services as your participation within this 
Risks: Some topics covered during the session may be sensitive, but you are not obligated to reveal any information. At no points are you requested to reveal your specific condition. The focus remains on navigating and accessing services. If you wish to not answer a question, or feel uncomfortable answering a question, please let the researcher know. There is a mild risk as some potential emotional and psychological discomfort may be triggering. If a question triggers any distress, please inform the researcher, and if needed, please requests resource for assistance if experiencing any emotional or mental distress. This list can be provided digitally or as printed lists.

Anonymity: All responses are anonymous and coded. You can also request that certain responses not be included in the final project. All research data, including audio-recordings and any notes will be encrypted. Any hard copies of data (including any handwritten notes or USB keys) will be kept in a locked cabinet at Carleton University. Research data will only be accessible by the researcher and the research supervisor.

Data Disposal: Your recording audio will be destroyed after the transcription of this audio-video which will occurs within two weeks of completing this session. If you wish to have your data removed from the study, please do so within two weeks of completing this session. After two weeks, data will be coded, and original audio-recording will be, removed from the video components of card-sorted images, transcribed, and then destroyed.

If you would like a copy of the finished research project, you are invited to contact the researcher to request an electronic copy which will be provided to you.

This project was reviewed and cleared by the Carleton University Research Ethics Board B. If you have any ethical concerns with the study, please contact Dr. Bernadette Campbell, Chair, Carleton University Research Ethics Board by phone at 613-520-2600 ext. 4085 for CUREB B] or by email at ethics@carleton.ca). Clearance \# 110744

\section{Researcher contact information: information:}

Name: Juan Saavedra

School of Industrial Design

Carleton University

Email:Juansaavedra@cmail.carleton.ca

Do you agree to be audio-video recorded:

\section{Supervisor contact}

Bjarki Hallgrimsson

School of Industrial Design

Carleton University

Email:

bjarki.hallgrimsson@cmail.carle ton.ca Yes No

Do you agree to let the research to take hand written notes: Yes No

Signature of participant

Date

Signature of researcher

Date 


\section{Appendix B Oral Script for Card-Sorting Interview}

Thank you again for the voluntary participation in this study. For this session, we will be focusing on a visual sorting-card activity, which relies on a mix of visual cues and your organizing process of those visual elements. This session should take approximately 20-60 minutes max. During this session, you will be asked to sort the visual cards in response to the scenario provided, followed by questions in the form of a semi-structured interview.

Risk: Should you feel uncomfortable with the scenario or feel uncomfortable at any stage of this session, please let the researcher know. Considering the session might touch upon sensitive issues, you are reminded that you are not obligated to reveal any information you do not wish to share. Your participation in this study is completely voluntary. You can withdraw from the study at any time. All data collected within this session will be destroyed immediately upon your withdrawal, should you choose to do so.

Compensation: There is no compensation as participation is voluntary. However, there will be light snacks and refreshments available during this session.

Purpose: The purpose of this session to gain a better understanding on your decision-making process, within the LGBTQ+ communities and to understand how your experiences can inform and improve gaps in social service design and delivery using design research methods.

Task: To begin, you will be given a set of sorting cards - a total of 15 , which feature a mix of concepts regarding daily activities. In addition, you will be given a set of blank cards used for later. Feel free to take as much time as you need to review the entire set. Using these sorting-cards, organize them into categories or themes in response to the scenario provided. You are not obligated to use all the cards within the set of 15 , simply choose which cards you feel are relevant. With the use of the blank cards, you may provide titles of each of the categories to help you organize. You will be also asked to use the blank cards to add additional concepts you feel are missing or needed to effectively respond to the scenario presented.

During this process, the visual sorting-cards will be video-recorded, as well as audio-recording to capture thinking processes performed aloud, as well as interview question responses. The video record will focus solely on the visual sorting-cards and may capture your hand movements. No facial features will be captured. Should you wish to be processed without any video-audio record, please let the researcher know. Differing the use of video-audio recording will not exclude you from participating in the session; alternatively, the researcher will produce handwritten notes of your session with your consent. 
If at any point you do not understand the instructions provided to you, the researcher will be glad to explain them again, simply ask for a reminder.

Once you have completed organizing the set of cards into themes, the researcher will ask you to explain and discuss your categories and themes.

Before we begin, do you have any questions? (If none, begin Q1)

\section{After reading the Oral Script to participants}

Q1: screener question is asked: Do you identify as a service user or service provider?

If, service user, proceed to scenario 1 , if service provider, proceed to scenario, proceed with follow up question: what is your professional title? Then move onto Scenario 2.

\section{Scenario 1 (S1) - For Service Users}

You have, just been diagnosed with an episodic disability / chronic health condition, using the visual sorting cards, what actions do you wish to take next after your diagnoses?

After Cards have been organized...

S1) Q2: Can you please share your process of how you organized the set of cards?

S1- Q3: Is there anything you would like to add to the set of cards?

S1- Q4: Was there any concepts taken out of set of cards?

S1- Q5: Do you learn anything from this activity?

S1- Q6: Do you have any additional thoughts, concerns, or comments that you would like to share in relation to this activity and scenario?

\section{Scenario 2 (S2)- For Service Providers}

An individual has just been diagnosed with an episodic disability/chronic health conditions and has been referred to your agency/non-profit services. Using the visual sorting cards, what actions do you wish for the service-user to take after the diagnose?

After Cards have been organized....

S2 Q2: Can you please share your process of how you organized the set of cards?

S2- Q3: Is there anything you would like to add to the set of cards?

S2- Q4: Was there any concepts taken out of set of cards?

S2- Q5: Do you learn anything from this activity?

S1- Q6: Do you have any additional thoughts, concerns, or comments that you would like to share in relation to this activity and scenario?

\section{***After the participants have answer the last question the oral}




\section{script follows for debrief $* * *$}

That's all the questions I have left. Thank you for participating within this study. If you have any questions, comments or concerns, please let me know.

If you are feeling distress or any discomfort from participating in this study, please let me know. If yes, please let the researcher know. Do you feel, you feel like you need to be referred to any services? If yes, let the researcher know.

[If short on participants] If you know anyone else who would be interested in participating, please refer them to me. 


\section{Appendix C Terms and Definitions Online Form + Consent}

\section{Carleton \\ U N I VE R S I T Y}

Canada's Capital University

\section{Design-Research and LGBTQ People Study}

Informing (Service)Design through LGBTQ+ Entrepreneurship, Activism and

(Invisible)Disabilities

Hello and thank you for your interest in this study. My name is Juan Saavedra, and I am a conducting research regarding the relationships between LGBTQ+ Communities, Entrepreneurship, Activism, Service Delivery and Invisible and Episodic Disabilities using Design Methods. This study is being conducted as part of my Master of Design thesis at Carleton University in Ottawa, Canada, under supervision of Bjarki Halligrimsson, School of Industrial Design and Dr. Xuan Thuy Nguyen, The Institute of Interdisciplinary Studies.

This online questionnaire-survey is should take between 10-15 minutes. This portion of this study is to better understand terms and definitions, and concerns happening within the LGBTQ+ communities in Ontario. The questions in this survey focus on the familiarity of terms and definitions, as well as their own interpretations and concerns within your own community.

To be eligible for this study: you must be 18+ years, residing within Ontario, Canada and must self-identify with the LGBTQ+ communities, and living with an invisible disability, or either volunteer or work in a professional capacity as a service provider. Participation within this study is voluntary.

By clicking "OK" and proceeding with the survey you are consenting to the collection of your responses and the conditions in which they are collected. All data is collected anonymously, without IP (internet Protocol) tracking.Your data will be stored and protected by Survey Monkey in Ontario, Canada, but may be disclosed via a court order or data breach. In summary, all answers are kept confidential and anonymous.

Risk: There is a mild risk involved as topics involve sensitive topics such as disability, navigating social service, and concerns about LGBTQ+ communities

Should you wish to withdraw from the survey during the questionnaire, feel free to close the browser at any moment. Closed and exited browsers will not retain any information. Answer boxes can be left unanswered, should you feel uncomfortable answering a question. Unanswered boxes will be interpreted as "I prefer not to answer." Your data can only be collected once you click on the "Submit" button at the end of the questions.

I am hoping that members of the LGBTQ+ communities will be interested in this study considering the limited studies in LGBTQ+ populations in relation to the field of Design and its capacity to improve Service and Program Delivery.

Please feel free to forward this questionnaire to any other LGBTQ+ peers, colleagues or service users or providers interested in participating. Should you have any questions, concerns or comments, I can be contacted through email: juansaavedra@cmail.carleton.ca

This project was reviewed and cleared by the Carleton University Research Ethics Board B. If you have any ethical concerns with the study, please contact Dr. Bernadette Campbell, Chair, Carleton University Research Ethics Board by phone at 613-520-2600 ext. 4085 for CUREB B] or by email at ethics@carleton.ca Clearance \# 110744.

Thank you for your participation. 
For the sake of reducing page count, this appendix has been modified to simplify the design of the survey. Questions 4 to 23 follow the format below exploring each term by first using a sliding bar for familiarity followed by an open text box for personal interpretation. Terms Used within this method were as follows: Design-Thinking, Design-Activism, Activism, Social Innovation, Social Enterprise, Disability, Episodic Disability, Invisible Disability, Accessibility, and ODSP. 
Using the sliding bar, how familiar are you with the term:

25. When considering LGBTQ+ community/communities in Ontario, Canada, what do you believe are the most concerning issues facing it?

24. When considering the LGBTQ+ community/communities, in the context of Ontario, Canada, what do you believe to be the most concerning issues?

26. Are you interested in participating further in this study? If yes, please provide an email address for the researcher to contact you. If not, please click the "Submit" button. Thank you for your participation!

As a reminder, no information will be collected or used until you click "Submit." You have the option to review your answers by scrolling back. Please review your answers. All submissions are final and there are no identifiers to identify you. If you have any hesitation about submitting, you can close the browser knowing that none of your information has been collected. Again, thank you for your participation! 


\section{Appendix D Participant Response Email Template}

\section{Email Invitation Response:}

This script is intended for those to who contact the researcher through email regarding the study:

Hello,

Thank for contacting me regarding the Design-Research and LGBTQ People Study. I am hoping that members of the LGBTQ+ communities will be interested considering the limited studies in LGBTQ+ populations in relation to the field of Design and its capacity to Social Service and Program Delivery while considering the complexities of sub-cultures such as Activism, Entrepreneurship and Invisible Disabilities.

To be eligible for this study: you must be $18+$ years of age; residing within Ontario, Canada; and must selfidentify with the LGBTQ+ communities. You must either be living with an invisible disability or volunteer/work in a professional capacity as a service provider for these respective communities. Participation in this study is voluntary.

Risk: There is a mild risk involved as topics involve sensitive topics such as disability, navigating social service, and concerns about LGBTQ+ communities. Participation is voluntary, and may select which activities to participate, you can volunteer to contribute in all, one or mix of design related activities listed below:

Online-Questionnaire: This may take up to 10-15 minutes. This task requires to identify terms and share how to affiliate with LGBTQ communities: Click Here: surveymonkey.com/r/DesignResearchLGBTQ

Photo-Voice: This may take up to 20-60 minutes. This activity involves taking original photos regarding expressions of inclusion and exclusion, and describe the photos To participate, please click here:

surveymonkey.com/r/DesignResearchLGBTQ-Photo

Card-Sorting and Semi-Structured Interview: This may take up to 20-60 minutes. This activity involves organizing a deck of cards, followed by a recorded semi structured interview, non-recorded option is available. This activity can only be done in person. Please reply to the researcher to volunteer your participation. The following dates are available:

(Insert List of dates available which are TBD in relation to ethics date approval)

If none of these dates are convenient, please let me know which days would work in your favor and l'll try to my best to accommodate.

If you have any thoughts, questions or concern please don't hesitate to contact the research at juansaavedra@cmail.carleton.ca 


\title{
Appendix E Photo-Voice Online Form and Consent Form
}

\section{Design-Research and LGBTQ People Study}

\author{
Photo Journal / Photovoice on LGBTQ Inclusion and Exlcusion Through Images \\ Hello and thank you for your interest in this study. My name is Juan Saavedra, and I am a conducting \\ research regarding the relationships between LGBTQ+ Communities, Entrepreneurship, Activism, \\ Service Delivery and Invisible and Episodic Disabilities using Design Methods. This study is being \\ conducted as part of my Master of Design thesis at Carleton University in Ottawa, Canada, under \\ supervision of Bjarki Halligrimsson, School of Industrial Design and Dr. Xuan Thuy Nguyen, The Institute \\ of Interdisciplinary Studies.
}

This online activity will take between 20-60 minutes. This portion of this study is to better understand the experiences of LGBTQ people using images. This method is known as Photovoice, in other instances, it can be sometimes be called Photo Journaling. This purpose of this method is to provide unique insights that are rooted in the lived experiences of the communities using this method.

Task: The prompts of this segment of study focus on experiences of inclusion and exclusion, and what these experiences entail. Participants are requested to reflect on places of inclusion and exclusion. Using their smart-phone devices / tablets, participants are asked to take photos of places and objects they interpret as inclusive and exclusive. Should you wish to send photos of people, it is implied you have obtained consent. All images must be your own. Participants can then upload their images through this online form.

To be eligible for this study: you must be 18+ years of age; residing within Ontario, Canada; and must self-identify with the LGBTQ+ communities. You must either be living with an invisible disability or volunteer/work in a professional capacity as a service provider for these respective communities. Participation in this study is voluntary.

By clicking "OK" and proceeding with the survey you are consenting to the collection of your responses and the conditions in which they are collected. All data is collected anonymously, without IP (Internet Protocol) tracking.Your data will be stored and protected by Survey Monkey in Ontario, Canada, but may be disclosed via a court order or data breach. In summary, all answers are kept confidential and anonymous

Risk: There is a mild risk involved as topics involve sensitive topics such as disability, navigating social service, and concerns about LGBTQ+ communities

Should you wish to withdraw from the survey during the questionnaire, feel free to close the browser at any moment. Closed and exited browsers will not retain any information. Answer boxes can be left unanswered, should you feel uncomfortable answering a question. Unanswered boxes will be interpreted as "I prefer not to answer." Your data can only be collected once you click on the "Submit" button at the end of the collection form.

I am hoping that members of the LGBTQ+ communities will be interested in this study considering the limited studies in LGBTQ+ populations in relation to the field of Design and its capacity to improve Service and Program Delivery.

Please feel free to forward this questionnaire to any other LGBTQ+ peers, colleagues or service users or providers interested in participating. Should you have any questions, concerns or comments, I can be contacted through email: juansaavedra@cmail.carleton.ca

This project was reviewed and cleared by the Carleton University Research Ethics Board B. If you have any ethical concerns with the study, please contact Dr. Bernadette Campbell, Chair, Carleton University Research Ethics Board by phone at 613-520-2600 ext. 4085 for CUREB B] or by email at ethics@carleton.ca Clearance \# 110744.

Thank you for your participation. 
2. Do you live in Ontario, Canada?

Yes

No

3. Please answer to which best describes you. You can select more than one option. Or give a personal response in the text-box provided.

I I am a social service provider within an LGBTQ+ affiliated organization.

I I access social services at an LGBTQ+ affiliated organizations.

Other (please specify)I identify in a professional capacity not listed above, and/or, identify in a combination of capacities not mentioned above. If so, please specify.

(a)

4. Using a smart-phone or tablet device, capture and submit an image(s) that you feel represents the concept of Inclusion.

To participate, you can upload up a maximum of 5 images and a minimum

Choose File No file chosen

5. Using the text box below, please describe why you chose the image(s) you submitted.

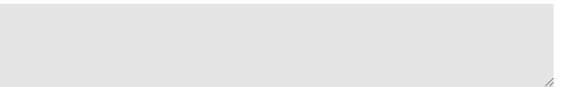

6. Using a smart-phone or tablet device, capture and submit an image(s) that you feel represents the concept of Exclusion.

To participate, you can upload up a maximum of 5 images and a minimum 1 image.

Choose File

No file chosen

7. Using the text box below, please describe why you chose the images you submitted

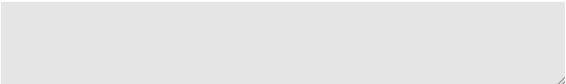

8. Do you wish for your images to be credited within the final study? If yes, provide desired name to be attributed towards images. If no, simply click on the "Done" button to submit all answers. 


\section{Appendix F Recruitment Email for Service Providers and Initiation of Study}

Dear <Insert Name of Person/Organization>

I am seeking volunteer participants for my master thesis study.

My name is Juan Saavedra, and I am a conducting research regarding the relationships between LGBTQ+ Communities, Entrepreneurship, Service Delivery and Invisible and Episodic Disabilities using Design Methods. This study is being conducted as part of my Master of Design thesis at Carleton University in Ottawa, Canada, under supervision of Bjarki Hallgrimsson, School of Industrial Design and Dr. Xuan Thuy Nguyen, The Institute of Interdisciplinary Studies.

I am reaching out to your organization because of your (insert inclusion criteria of study) If there is any interest in contributing towards my study through volunteering in an interview session, or another design related activity, please let me know. I am hoping that members of the LGBTQ+ communities will be interested considering the limited studies in LGBTQ+ populations in relation to the field of Design and its capacity to improve Service and Program Delivery. Session can be scheduled around your convenience. Please feel free to forward this email message to any other LGBTQ+ peers, colleagues or service users interested participating. I can be contacted through email: juansaavedra@cmail.carleton.ca with any questions.

You can participate in all, or one or a mix of the following activities:

The card-sorting and semi-structured interview portion of the study will take place between (Upon approval of ethics boards 2019.) Each session will require approximately 20 min - 60max minutes. Participants will be asked to sort a set of cards and think aloud regarding their process. The goal of this is to understand the participants' thinking process regarding the scenarios presented.

Photovoice Activity / Photo Journaling: Using your smart phone device, you will be asked to capture a set of images that describe your interpretation of the prompts provided by the researcher. Specifically, this activity will focus on your interpretations of inclusion and exclusion. Will take about 20 min - 60 max minutes.

The online questionnaire-survey can be accessed through the link provided: (insert link). This segment focuses on terms and definitions and other concerns for LGBTQ communities. This questionnaire should take roughly 10-15 minutes.

Information is collected through our online platforms are done anonymously and does not track IP (internet protocol) through the duration of the online questionnaire survey. our data will be stored and protected by Survey Monkey in Ontario, Canada, but may be disclosed via a court order or data breach in summary, all answers are kept confidential and anonymous.

If you have any ethical concerns with the study, please contact Dr. Bernadette Campbell, Chair, Carleton University Research Ethics Board-B (by phone at 613-520-2600 ext. 4085 or via email at ethics@carleton.ca). Clearance \# 110744

Thank you, Juan Saavedra, MDes Candidate Carleton University, School of Industrial Design 


\section{Appendix G Visual Sorting Card- Activity Deck - Set Up}

These Sorting-Cards feature concepts, with word and illustrations, loosely related to everyday activities. Some of the concepts have been informed by casual conversations with LGBTQ+ people living with episodic disabilities, entrepreneurs and activists; this method is similar to co-design The lists of concepts included: Medical Specialists, Travel, Co-workers, Leisure, Medication, Employment, Food, Social Worker, School, Family Doctor, Volunteering, Housing, Family, Friends and Support Groups.

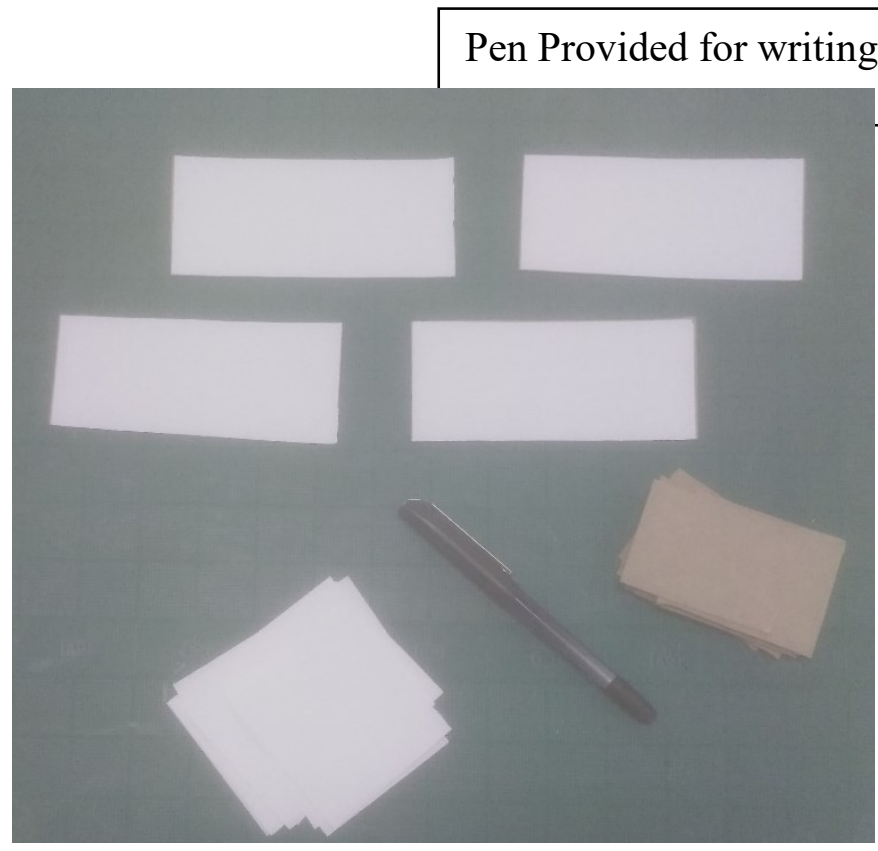

Sorting-cards faced down.

Rectangle blank cards

Used to give titles for

Blank Square cards given for participants to add toward the sorting card deck, should they wish to do so.

\section{Mock-up of how the visual sorting activity will be executed.}
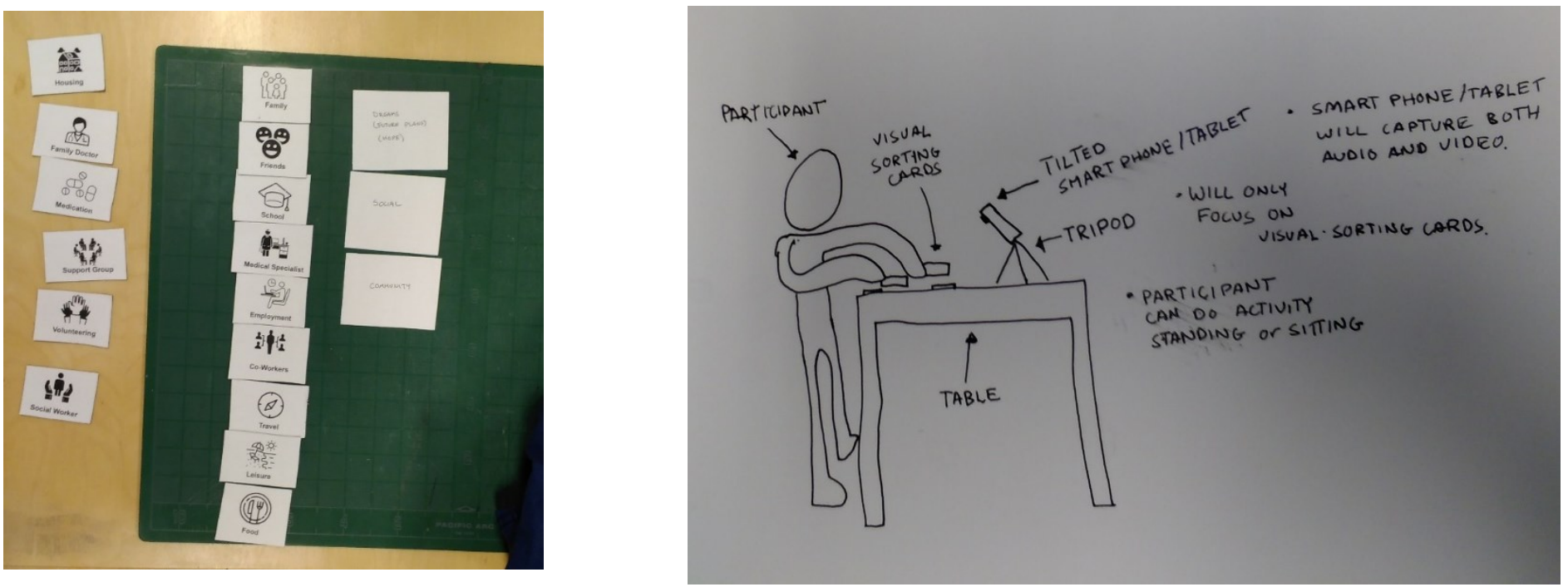


\section{Appendix H - Factor Analysis Source Code}

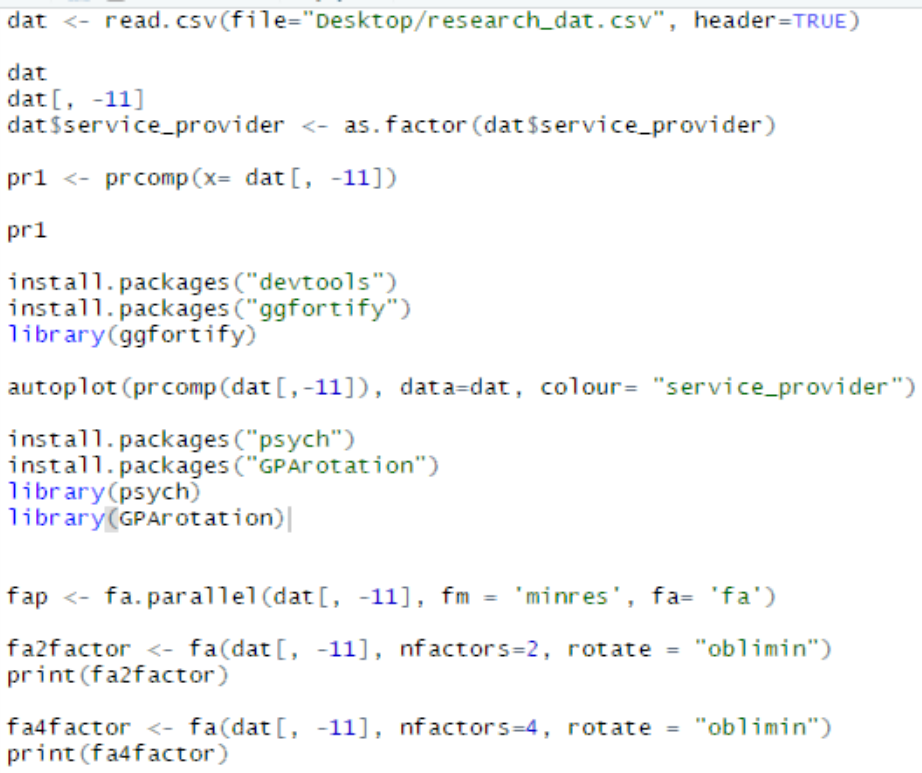

Appendix I) Final Sorts of All Participants Users and Providers

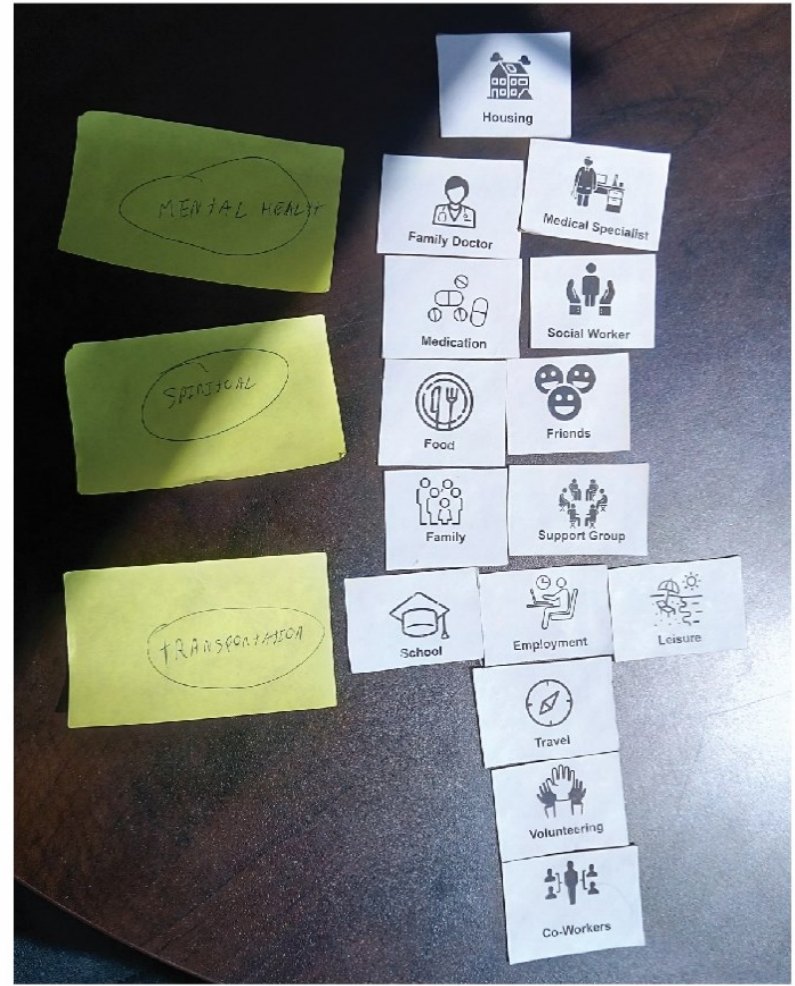

P1 - SERVICE PROVIDER

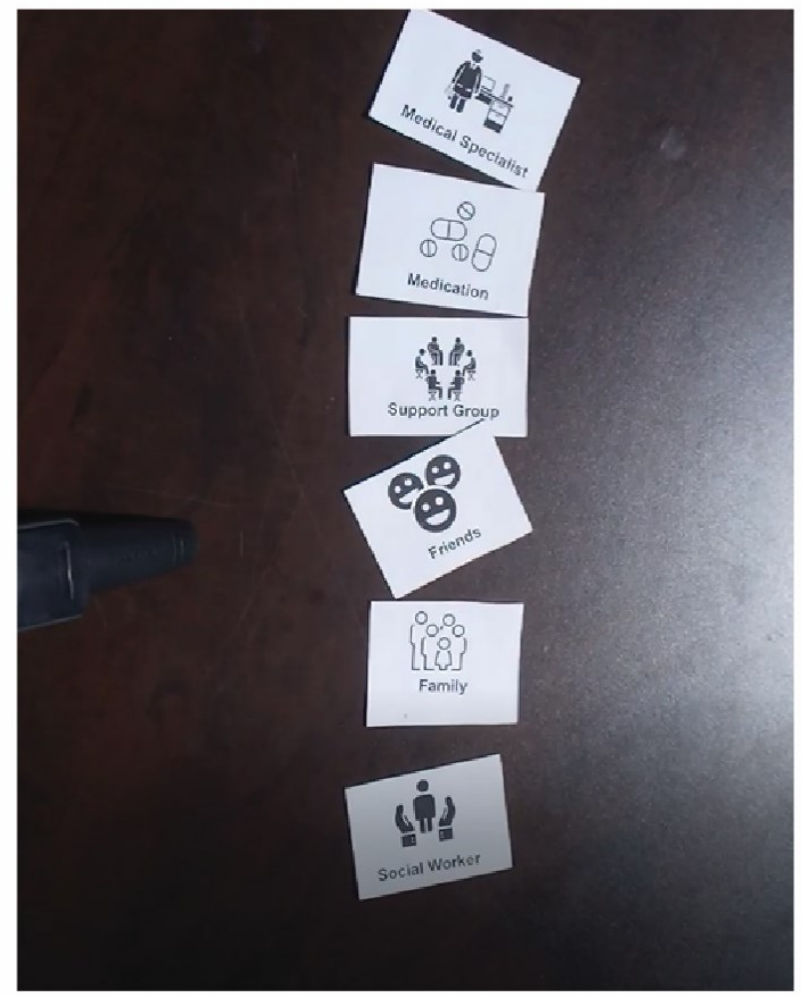

P2- SERVICE PROVIDER 


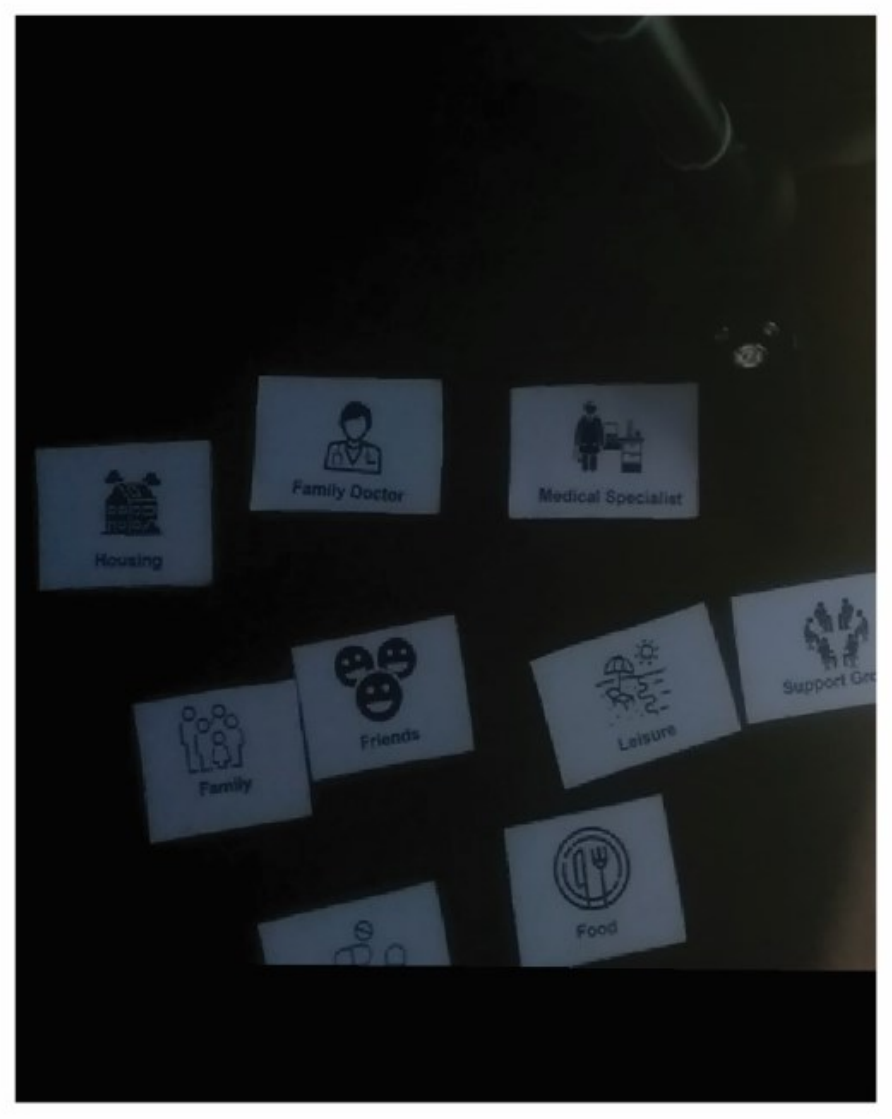

P3- SERVICE PROVIDER

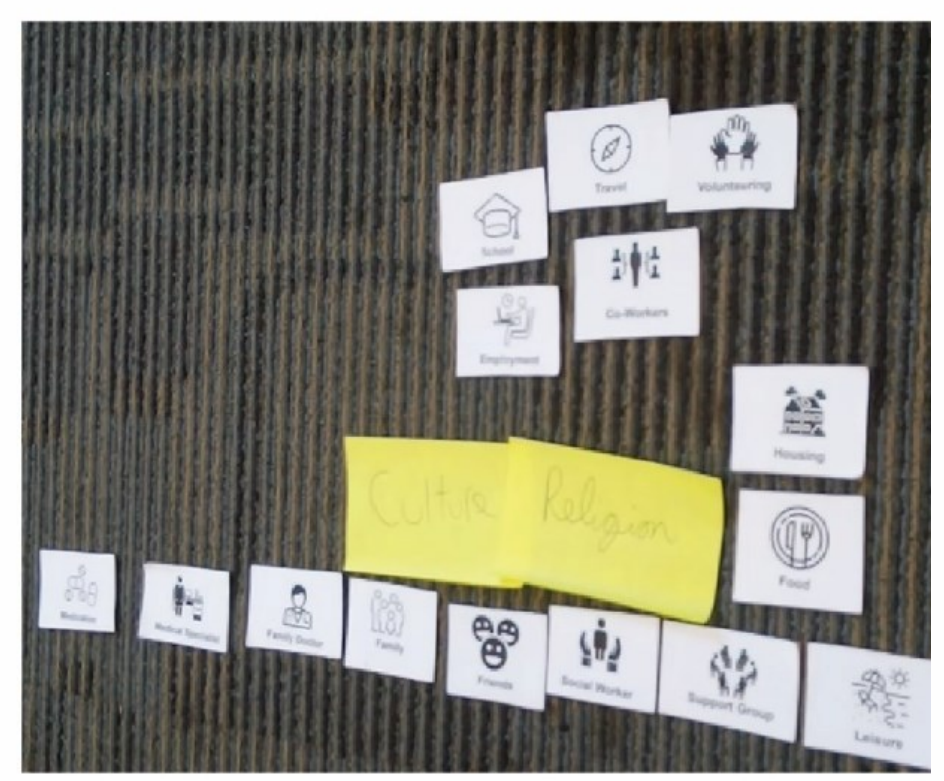

P5 - SERVICE PROVIDER

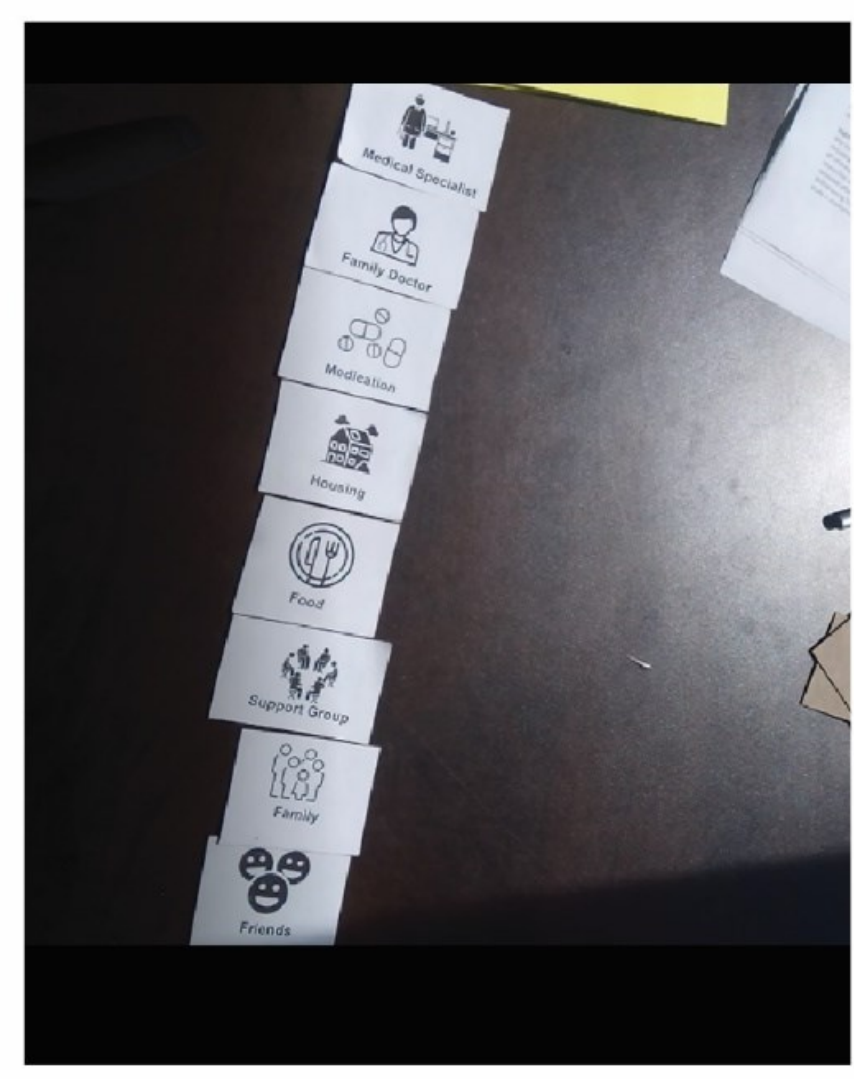

P4- SERVICE PROVIDER

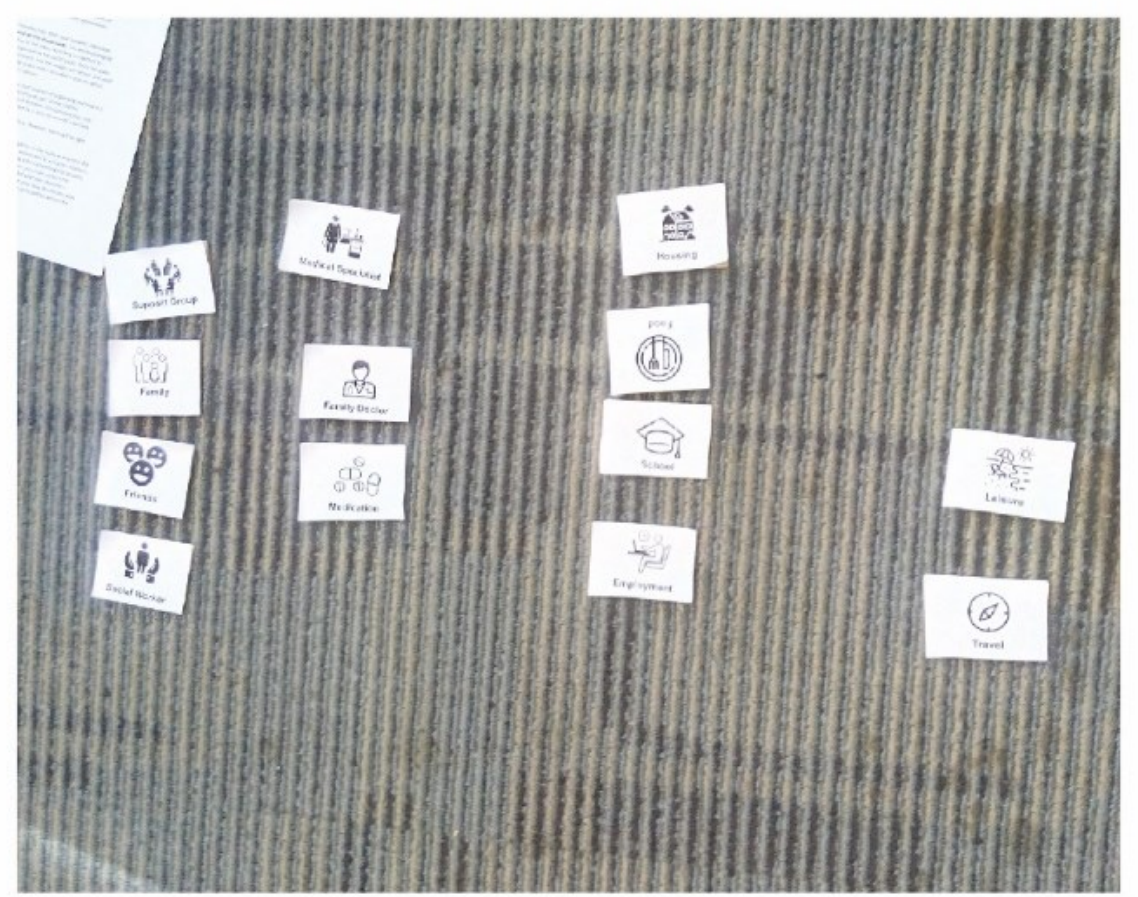

P6 - SERVICE PROVIDER 


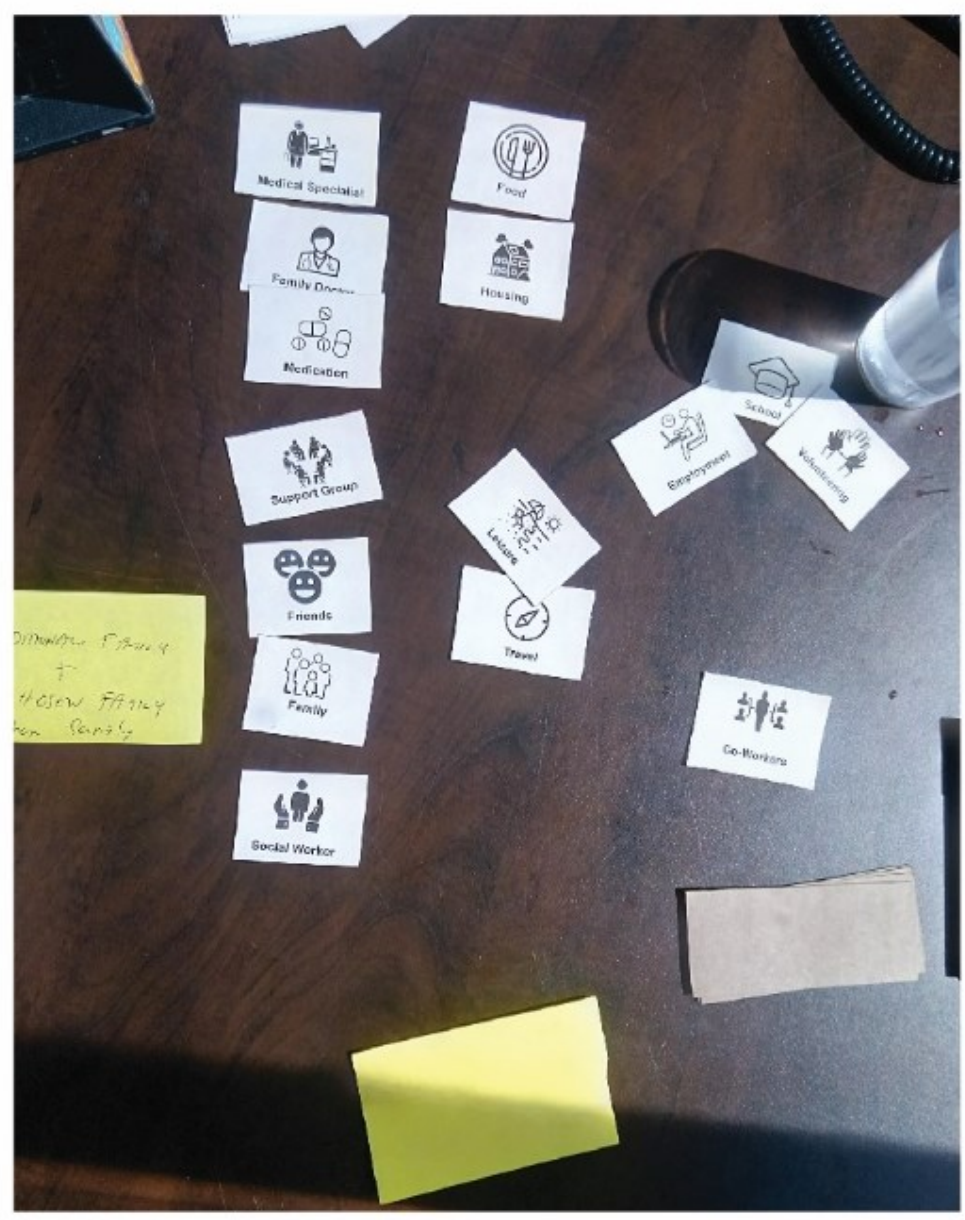

\section{: PROVIDER}

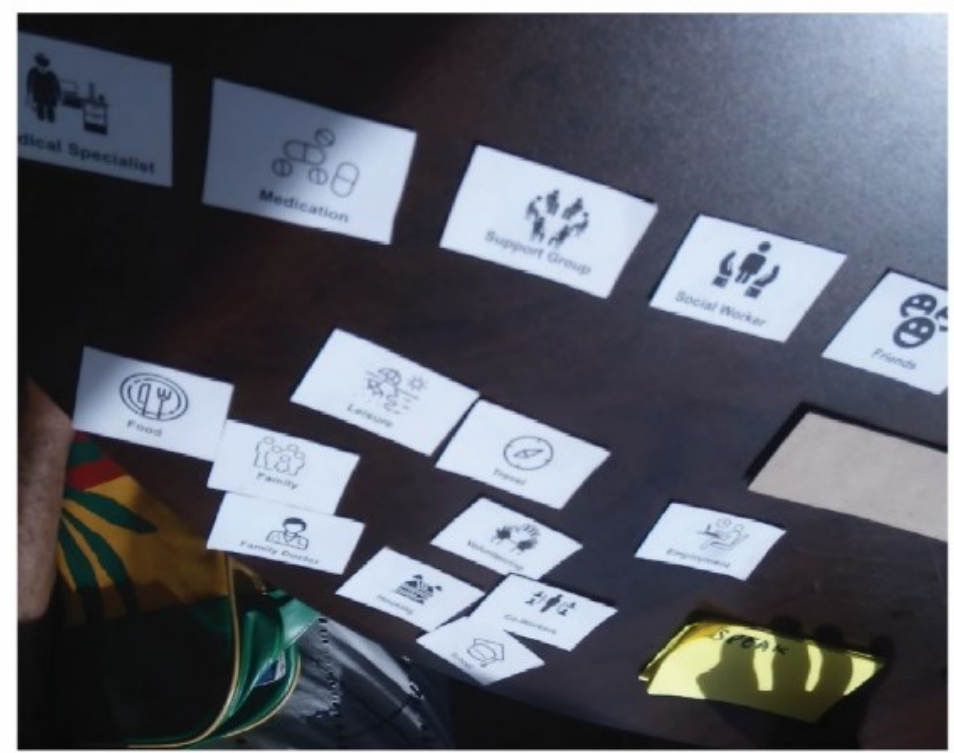

P1 - SERVICE USER

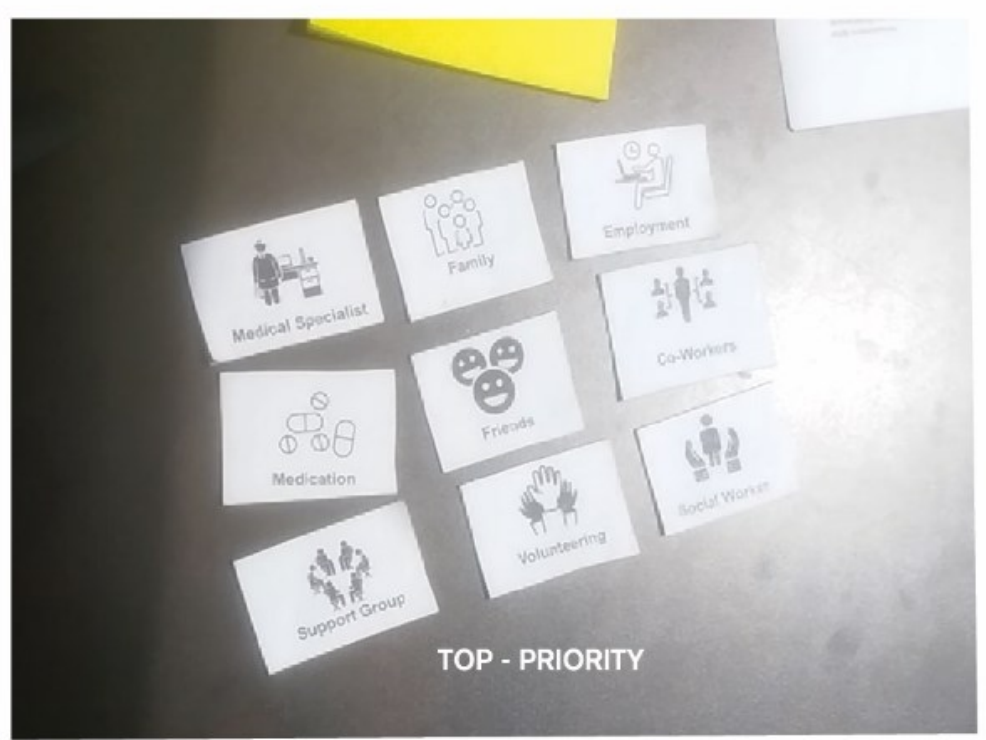

P2 - SERVICE USER 


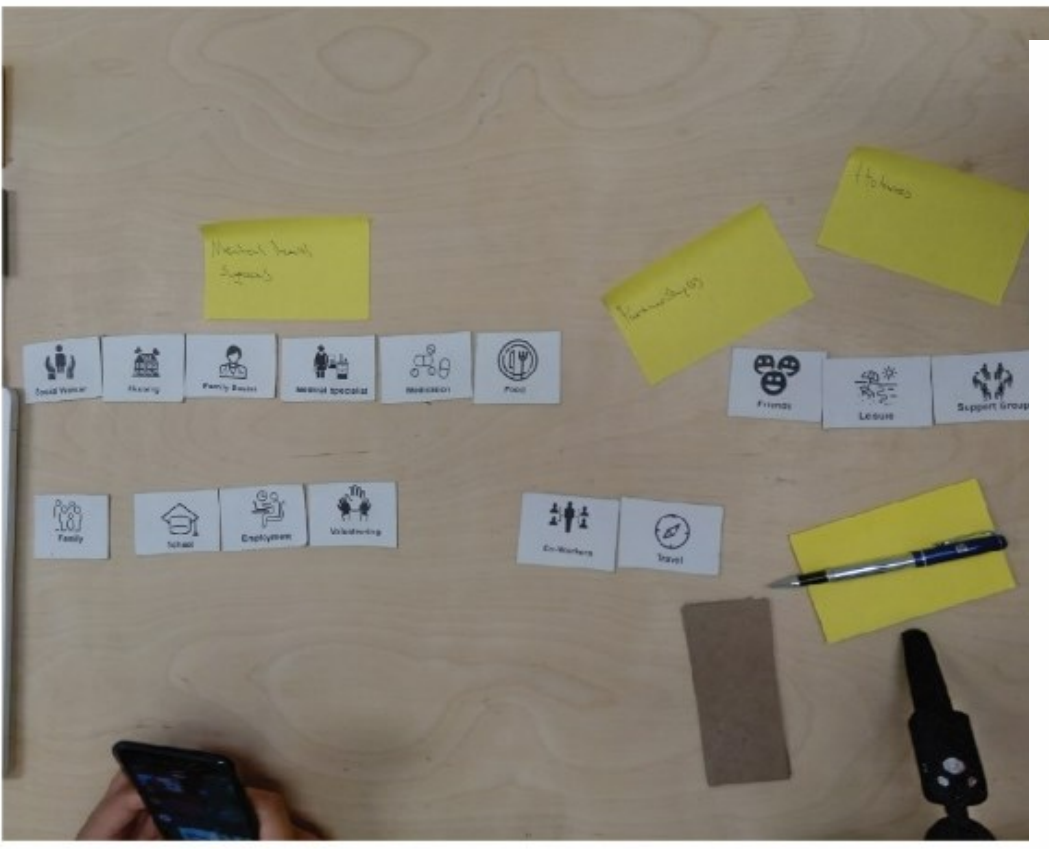

P3- SERVICE USER

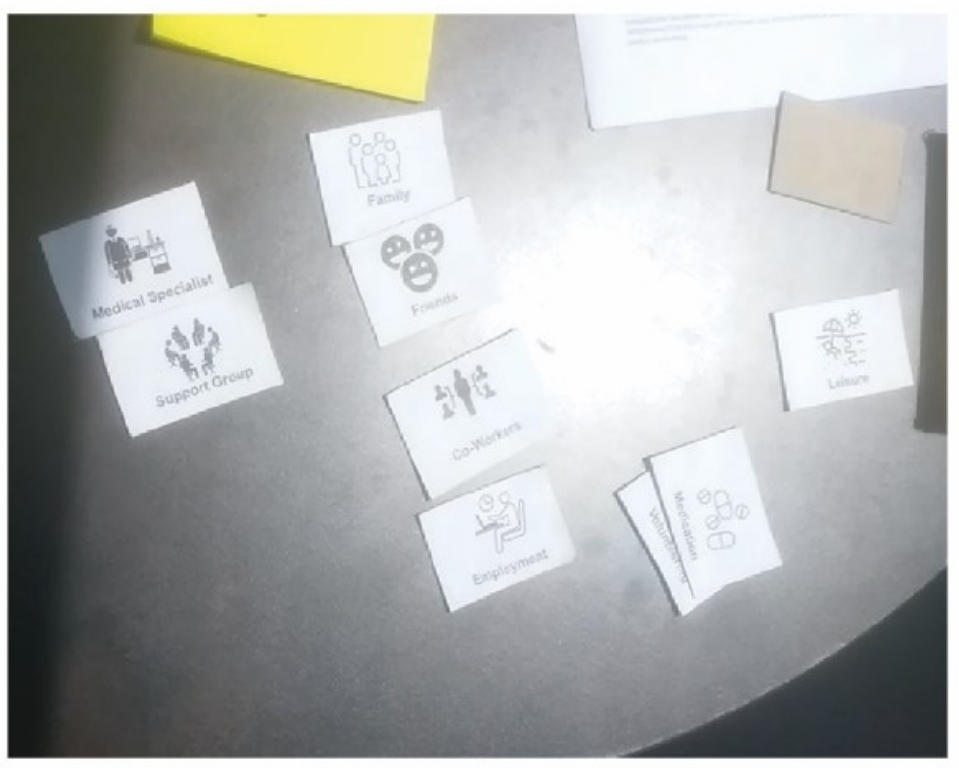

P4- SERVICE USER

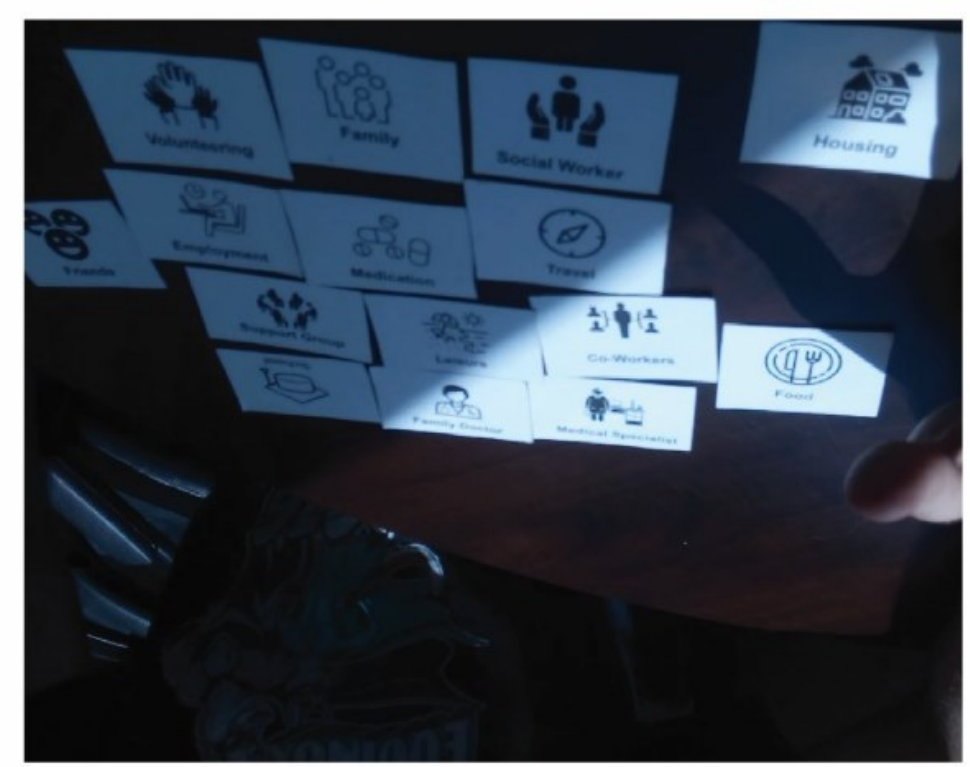

P5- SERVICE USER

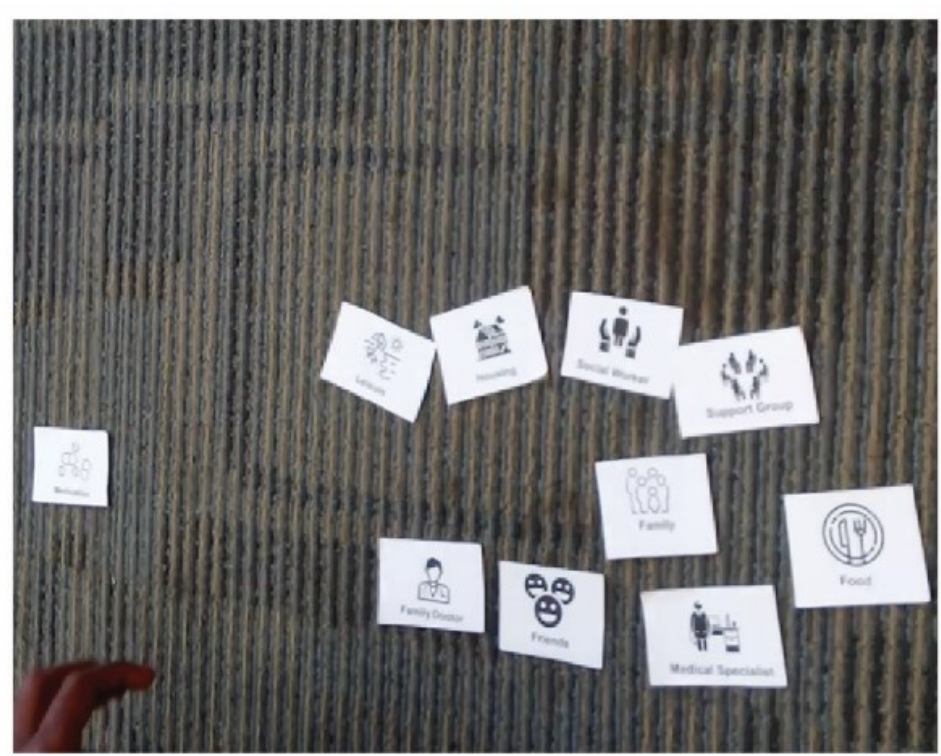

P6- SERVICE USER

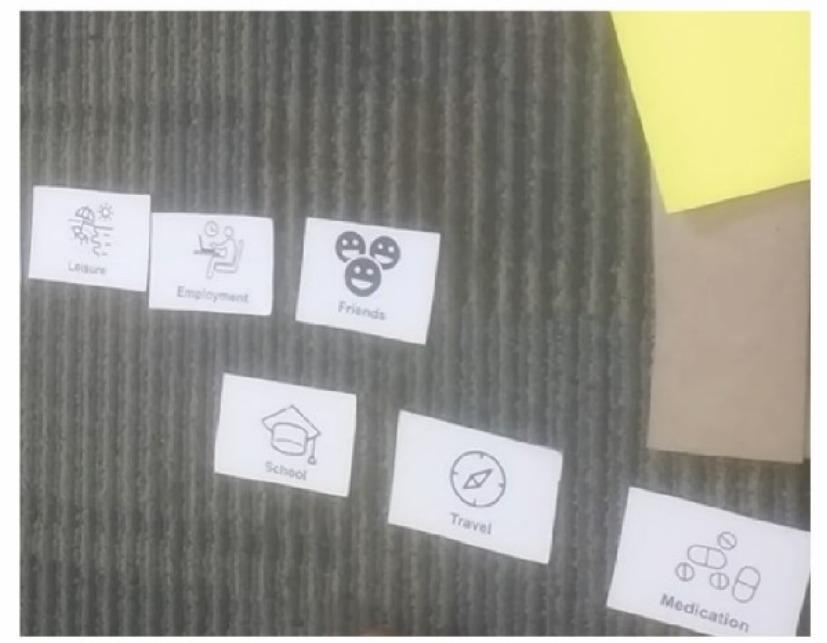




\section{References}

Ahmed, M.M. (2018) Minorities Designers - Lead the change towards social responsibility. In Citizen Designer: Perspectives on Design Responsibility edited by Heller, S. and Vienne, V. $2^{\text {nd }}$ edition. Allworth.

Anderson, D. (2014) Graphic Design and Entrepreneurship: The Rise of the Design Entrepreneur. Creativity and Society 22.

Alvord, S. H, Brown, L., D. W. Letts, C. (2004) "Social Entrepreneurship and Societal Transformation: An Exploratory Study." The Journal of Applied Behavioral Science. Vol 40, Issue 3, pp. 260 - 282. https://doi.org/10.1177/002188630426684

Armstrong, H. (2009) Graphic Design Theory Readings from The Field. Princeton Architectural Press New York.

Aronoff, U., \& Gilboa, A. (2015) Music and the closet: The roles music plays for gay men in the "coming out" process. Psychology of Music, 43(3), 423-437. https://doi.org/10.1177/0305735613515943

Austin, J., Stevenson, H., \& Wei-Skillern, J. (2006). Social and Commercial Entrepreneurship: Same, Different, or Both? Entrepreneurship Theory and Practice, 30(1), 1-22. https://doi.org/10.1111/i.1540-6520.2006.00107.x

Barclay, L.A., Markel, K.S., Goldberg, C., and Simon, M. (2014) Persons with Disabilities and Entrepreneurship: Barriers and Opportunities Academy of Management Proceedings, 2014:1

Balsam, K. F., Molina, Y., Beadnell, B., Simoni, J., \& Walters, K. (2011). Measuring multiple minority stress: the LGBT People of Color Microaggressions Scale. Cultural diversity \& ethnic minority psychology, 17(2), 163-174. doi:10.1037/a0023244

Bardhoshi, G., Grieve, K., Swanston, J., Suing, M. and Booth, J. (2018), Illuminating the On-Campus Experience of LGBTQ Students Through Photovoice. Journal of College Counseling, 21: 194-209. doi:10.1002/jocc.12103

Binnie, J (2014) Relational Comparisons and LGBTQ Activism in European Cities. International Journal of Urban and Reginal Research. Volume 38.3

Block, G. (2019). Changing disability definition, a dangerous mistake that will harm thousands. The Star News. Retrieved from https://www.thestar.com/opinion/contributors/2019/04/08/changing-disabilitydefinition-a-dangerous-mistake-that-will-harm-thousands.html 
Boisvert, N and Xing, L. (2018). Ontario PCs to update disability requirements, allow welfare recipients to earn more money. CBC News. Retrieved from: https://www.cbc.ca/news/canada/toronto/doug-ford-social-assistance-plan-1.4915315

Bonsiepe, G. (2006) Design and Democracy. Design Issues Volume 22, Number 2.

Branchik, B. J. (2002). Out in the Market: A History of the Gay Market Segment in the United States. Journal of Macromarketing, 22(1), 86-

97. https://doi.org/10.1177/027467022001008

Branchik, B. J (2007) Queer Ads: Gay Male Imagery in American Advertising, Consumption Markets \& Culture, 10:2, 147-158, DOI: $\underline{10.1080 / 10253860701256232}$

Brotman, S., Ferrer, I., Sussman, T., Ryan, B., \& Richard, B. (2015). Access and equity in the design and delivery of health and social care to LGBTQ older adults: A Canadian perspective. In N. A. Orel \& C. A. Fruhauf (Eds.), The lives of LGBT older adults:

Understanding challenges and resilience (pp. 111-140). Washington, DC, US: American Psychological Association. http://dx.doi.org/10.1037/14436-006

Brown, T. (2009) Change by Design How Design Thinking Transforms Organizations and Inspires Innovation. Harper Business.

Buck-Coleman, A., McDonald, A., \& Bidle, M. (2011) Bridging Diversity: Ethical Considerations in Design Education, Iridescent, 1:1, 12-

21, DOI: $10.1080 / 19235003.2011 .11782238$

Buchanan, R (1992) Wicked Problems in Design Thinking Source. Design Issues, Vol. 8, No. 2, (pp. 5-21)

Butcher, J, Dobson, C, Atkinson, A, Clayton, A \& Couzins-Scott, E. (2016) Sex and Cancer: opening difficult therapeutic conversations and challenging assumptions using Design Activism-language outside the comfort zone, Sexual and Relationship

Therapy, 31:4, 493-509, DOI: 10.1080/14681994.2016.1190815

Cairns, G. (2017) Can Design Inform Effective Social Innovation? The Design Journal, 20:6, 725-734, DOI: 10.1080/14606925.2017.1370658

Canadian HIV/AIDS Legal Network (2019) THE CRIMINALIZATION OF HIV NON-

DISCLOSURE IN CANADA: CURRENT STATUS AND THE NEED FOR CHANGE. AIDS LaW, Retrieved from: http://www.aidslaw.ca/site/the-criminalization-of-hiv-non-disclosurein-canada-report/?lang=en 
Canadian Press, the (2018) Modern sex-ed curriculum has huge support among Ontario parents and students, consultations show. CBC News. Retrieved from:

https://www.cbc.ca/news/canada/toronto/ontario-sex-ed-consultations-1.4949832

CATIE.ca, Canadian AIDS Treatment Information Exchange. (2016) The Basics. Retrieved from: https://www.catie.ca/en/basics Revised 2019.

Çeviker-Çınar, G., Mura, G., \& Demirbağ-Kaplan, M. (2017) Design Thinking: A New Road Map in Business Education, The Design Journal, 20:sup1, S977-S987, DOI:

10.1080/14606925.2017.1353042

Çetin, Ö. \& Aryana, B. (2015) An Analysis of The Influence of Design Activism and Socially Responsible Design Approaches on Design Education. 10.13140/RG.2.1.1870.4809.

Chamberlain, P. \& Partridge, R. (2017) Co-designing co-design. Shifting the culture of practice in healthcare, The Design Journal, 20:sup1, S2010-S2021, DOI:

10.1080/14606925.2017.1352720

Colpitts, E., \& Gahagan, J. (2016). The utility of resilience as a conceptual framework for understanding and measuring LGBTQ health. International Journal for Equity in Health, 15, 60. http://doi.org/10.1186/s12939-016-0349-1

Cooper, R. (2005) Ethics and Altruism: What Constitutes Socially Responsible Design? Design Management Review.

Clare, E. (2015) Exile and Pride: Disability, Queerness, and Liberation, Duke University Press

Clarke, A.J. (2013) “Actions Speak Louder", Design and Culture, 5:2, 151168, DOI: $\underline{10.2752 / 175470813 \times 13638640370698}$

Crawley, M. (2019) Scrapping sex-ed curriculum doesn't violate Constitution, Ford government argues. CBC News. Retrieved from:

https://www.cbc.ca/news/canada/toronto/doug-ford-social-assistance-plan-1.4915315

Crisp, D.G. (2009) Discourse This! Designers and Alternative Critical Writing, Design and Culture, 1:1,105-120, DOI: 10.2752/175470709787375850

Cross, N. (2006). Designer-ly Ways of Knowing. Springer. 10.1007/1-84628-301-9. 
Daley, A. E., \& MacDonnell, J. A. (2011). Gender, sexuality and the discursive representation of access and equity in health services literature: implications for LGBT communities. International Journal for Equity in Health, 10, 40.

http://doi.org/10.1186/1475-9276-10-40

Denison, P. (2011). Design futuring: Sustainability, ethics and new Practice/Design activism: Beautiful strangeness for a sustainable world. Journal of Design History, 24(4), 397-400. doi: http://dx.doi.org.proxy.library.carleton.ca/10.1093/jdh/epr032

Dhaenens, F., \& Burgess, J. (2018). 'Press play for pride': The cultural logics of LGBTQthemed playlists on Spotify. New Media \& Society. https://doi.org/10.1177/1461444818808094

DeVita, T., Bishop, C., \& Plankey, M. (2018) Queering medical education: systematically assessing LGBTQI health competency and implementing reform, Medical Education Online, 23:1, DOI: $\underline{10.1080 / 10872981.2018 .1510703}$

Dimarco, P. (2011) Issue-Based Design Education: Changing the way future communication designers are taught in a global society, Iridescent, 1:1, 7281, DOI: 10.1080/19235003.2011.11782245

Dong, H. (2010) Strategies for teaching inclusive design, Journal of Engineering Design, 21:2-3, 237-251, DOI: 10.1080/09544820903262330

Song, D, and Lou, Y. (2016) Design Activism: Action research as an approach when design meets social innovation, In: Wong, Wendy Siuyi; Kikuchi, Yuko \& Lin, Tingyi (Eds.). Making Trans/National Contemporary Design History [=ICDHS 2016 - 10th Conference of the International Committee for Design History \& Design Studies]. São Paulo: Blucher, 2016. ISSN 2318-6968, DOI 10.5151/despro-icdhs2016-03_018p. 284-290.

Duke, T.S (2011) "Lesbian, Gay, Bisexual, and Transgender Youth with Disabilities: A Meta-Synthesis" Journal of LGBT Youth Vol. 8, Iss. 1 https://doi.org/10.1080/19361653.2011.519181

Eckhert, E. (2016). A Case for the Demedicalization of Queer Bodies. The Yale Journal of Biology and Medicine, 89(2), 239-246.

EDEN Canada (n.d) About episodic disabilities Retrieved from: http://edencanada.ca/en/episodicdisabilities Episodic Disability Network (2015) Episodic Disability Tool Kit of Resources. Elbaz, G. (1995) Beyond anger: The activist construction of the AIDS crisis. Social Justice, 22(4), 43. 
Elsharkway, A. E. (2017) What is Critical Discourse Analysis (CDA)? The Second Literary Linguistics Conference (4-6 October 2017, Johannes Gutenberg-Universität Mainz)

Escobar, A. (2015) Transiciones: a space for research and design for transitions to the pluriverse, Design Philosophy Papers, 13:1, 13-

23, DOI: $10.1080 / 14487136.2015 .1085690$

Fassi, D. \& Meroni, A. \& Simeone, G. (2013). Design for Social Innovation as a form of Design Activism: An action format. Social Frontiers: The Next Edge of Social Innovation Research London

Featherman, C. (2016) Disability and discourse analysis, Critical Discourse Studies, 13:1, 137-139, DOI: 10.1080/17405904.2015.1105419

Fillis, I. \& Rentschler, R. (2010). The Role of Creativity in Entrepreneurship. Journal of Enterprising Culture (JEC). 18. 49-81. 10.1142/S0218495810000501.

Fischer, H. (1977) Gay Semiotics. Queer Cultural Centre. Retrieved from: http://www.queerculturalcenter.org/Pages/HalPages/GaySempg5.html

Fredriksen-Goldsen K. I. (2016). The Future of LGBT+ Aging: A Blueprint for Action in Services, Policies, and Research. Generations (San Francisco, Calif.), 40(2), 6-15.

Fritsch, K. (2016) Accessible. In Keywords for Radicals, The Contested Vocabulary of Late-Capitalists Struggle. 23-28. Edited by Fritsch, K., O'Connor, C., and Thompson, A.K. AK Press.

Fry, T. (2009) Design Futuring: Sustainability, Ethics and New Practice. Berg. Oxford International Publishing

Fuad-Luke, A. (2009) Design Activism: beautiful strangeness for a sustainable world. Earthscan from Routledge.

Foucault, M. (1977) Discipline and Punish: The Birth of the Prison. New York Vintage.

Foucault, M. (1978) The history of sexuality, volume 1: An introduction (R, Hurley Trans.) New York Vintage.

Foucault, M. (2003) Society Must be defended: Lectures at the College de France,19751976. New York, NY Picador.

Gheorghe, R. I, (2016) Public Awareness Campaigns: A Consumer-Orientated Perspective Regarding the Advertising on LGBTQ Minorities. $5^{\text {th }}$ World Conference on Business, Economics, Management, WCBEM. 
Ginder, W. and Byun, S. (2015), Past, Present, and Future of Gay and Lesbian Consumer Research: Critical Review of the Quest for the Queer Dollar. Psychol. Mark., 32: 821-841. doi: $10.1002 /$ mar.20821

Giwa S, and Greensmith C. J (2012) Race relations and racism in the LGBTQ community of Toronto: perceptions of gay and queer social service providers of color. Homosexual. 2012;59(2):149-85. doi: 10.1080/00918369.2012.648877.

GLSEN. (2018). Laws Prohibiting "Promotion of Homosexuality" in Schools: Impacts and Implications (Research Brief). New York: GLSEN.

Gould, D. (2009) The Pleasures and Intensities of Activism; in Moving Politics: Emotion and ACT UP's Fight Against AIDS (Chicago: University of Chicago Press, 181-212

Government of Canada (2019) Social Determinates of Health and Health Inequalities. Retrieved from: https://www.canada.ca/en/public-health/services/healthpromotion/population-health/what-determines-health.html

Greene, S., Ion, A., Kwaramba, G., Mwalwanda, M. , Caswell, J., Guzha, E., and Carvalhal, A., (2015) "Trust Me, It's Different": Experiences of Peer Case Management of Women Living with HIV in Ontario, Canada Volume 32, N. 1, pp. 5-210, Canadian Social Work Review Online. DOI https://doi.org/10.7202/1034144

Groot, A., \& Dankbaar, B. (2014). Does Social Innovation Require Social Entrepreneurship? Technology Innovation Management Review, 4(12).

Guffey, E. (2015) The Scandinavian Roots of the International Symbol of Access, Design and Culture, 7:3, 357-376, DOI: 10.1080/17547075.2015.1105527

Gray,M.L. (2009) Negotiating Identities/Queering Desires: Coming out Online and the Remediation of the Coming-out Story, Journal of Computer-Mediated Communication, Volume 14, Issue 4, 1 July 1162-1189, https://doi.org/10.1111/j.1083$\underline{6101.2009 .01485 . x}$

Greenspan, N.R, (2018) Evaluation Policy at AIDS Service Organizations: Managing Multiple Accountabilities. In, Seeing Red: HIV AIDS and Public Policy in Canada. Edited by Hindmarch, S., Orsini, M., and Gagnon, M. (2018). University of Toronto Press.

Grudin, R. (2010) Design and Truth. Yale University Press. Hamraie, A. (2016) Universal Design and the Problem of "Post-Disability" Ideology, Design and Culture, 8:3, 285-309, DOI: 10.1080/17547075.2016.1218714 
Hamraie, A., \& Fritsch, K. (2019). Crip technoscience manifesto. Catalyst: Feminism, Theory, Technoscience, 5(1), 1-34

Harris, S.P., Renko, M., and Caldwell, K. (2014) Social entrepreneurship as an employment pathway for people with disabilities: exploring political-economic and socio-cultural factors, Disability \& Society, 29:8, 1275-1290, DOI:

10.1080/09687599.2014.924904

Hackney, F. (2013) Quiet Activism and the New Amateur, Design and Culture, 5:2, 169193, DOI: $10.2752 / 175470813 \times 13638640370733$

Haddad, N., Li, J.S., Totten, S., McGuire, M. (2017) HIV in Canada-Surveillance Report, Can Commun Dis Rep 2018;44(12):324-32. https://doi.org/10.14745/ccdr.v44i12a03

Halberstam, J. (2011) The Queer Art of Failure. DukePress

Harper, G. W., Serrano, P. A., Bruce, D., \& Bauermeister, J. A. (2016). The Internet's Multiple Roles in Facilitating the Sexual Orientation Identity Development of Gay and Bisexual Male Adolescents. American journal of men's health, 10(5), 359-376.

doi:10.1177/1557988314566227

Health Canada (2009) HIV/AIDS and Disability: Final Report of the 4th International Policy Dialogue. Mister of Health. Retrieved from http://data.unaids.org/pub/report/2009/20091111 hiv and disability en.pdf

Heidaripour, M \& Forlano, L. (2018). Formgiving to Feminist Futures as Design Activism. $10.21606 /$ drs.2018.464.

Heller, S. (2013) Branding AIDS, In Design Cult, 25 Essay on Graphic Design Culture. DesignFile. Location 1053 - 1108, Kindle Edition.

Heller, S. and Vienne, V. (2003) Citizen Designer: Perspectives on Design Responsibility, $1^{\text {st }}$ edition. Allworth.

Heller, S. and Vienne, V. (2018) Citizen Designer: Perspectives on Design Responsibility, $2^{\text {nd }}$ edition. Allworth.

Hindmarch, S., Orsini, M., and Gagnon, M. (2018) Seeing Red: HIV AIDS and Public Policy in Canada. University of Toronto Press.

Hoen, E., Berger, J., Calmy, A., \& Moon, S. (2011). Driving a decade of change: HIV/AIDS, patents and access to medicines for all. Journal of the International AIDS Society, 14, 15. doi:10.1186/1758-2652-14- 
Hollibaugh, A and Weiss, M. (2015) Queer Precarity and the Myth of Gay Affluence New Labor Forum Vol 24, Issue 3, pp. 18 - 27, https://doi.org/10.1177/1095796015599414

Holtby, A., Klein, K., Cook, K., \& Travers, R. (2015). To be seen or not to be seen: Photovoice, queer and trans youth, and the dilemma of representation. Action Research, 13(4), 317-335. https://doi.org/10.1177/1476750314566414

Imrie, R and Luck, R. (2014) Designing inclusive environments: rehabilitating the body and the relevance of universal design, Disability and Rehabilitation, 36:16, 13151319, DOI: $10.3109 / 09638288.2014 .936191$

Income Security Advocacy Centre. (2019) Changes Ontario is Proposing to OW and ODSP. Retrieved from: http://incomesecurity.org/policy-advocacy/changes-ontario-isproposing-to-ow-and-odsp/

Institute of Medicine. (2011). The health of lesbian, gay, bisexual, and transgender (LGBT) people: Building a foundation for better understanding. Retrieved from http://www.iom.edu/

INTO (2018) Brand Innovators Research Finds Marketers, Agencies Too Often Miss Key Opportunities to Authentically Engage with LGBTQ Consumers Retrieved from https://www.prnewswire.com/news-releases/into-brand-innovators-research-findsmarketers-agencies-too-often-miss-key-opportunities-to-authentically-engage-withlgbtq-consumers-300614115.html

Julier, G. and Kaygan, H. (2013) Global Design Activism Survey, Design and Culture, 5:2,237-252, DOI: 10.2752/175470813X13638640370850

Julier, G (2013) From Design Culture to Design Activism, Design and Culture, 5:2, 215236, DOI: 10.2752/175470813X13638640370814

Kafer, A. (2013) Feminist, Queer, Crip. Indiana University Press; 1 edition

Kalle, L. (2006) Design Anarchy, In Graphic Design Theory Readings from The Field. Edited by Armstrong, H. Princeton Architectural Press New York., pg. 107.

Kattari, S.K, Walls, N.E., Whitfield, D.L., \& Magruder, L.L., (2017) Racial and Ethnic Differences in Experiences of Discrimination in Accessing Social Services Among Transgender/Gender-Nonconforming People, Journal of Ethnic \& Cultural Diversity in Social Work, 26:3, 217-235, DOI: 10.1080/15313204.2016.1242102

Kelleher, C. (2009) Minority stress and health: Implications for lesbian, gay, bisexual, transgender, and questioning (LGBTQ) young people, Counselling Psychology 
Quarterly, 22:4, 373-379, DOI: 10.1080/09515070903334995

Kimbell, L. (2009). The turn to Service Design, in Gulier, J. and Moor, L. (eds.), Design and Creativity: Policy, Management and Practice, Oxford: Berg.

Kimbell, L. (2012) Rethinking Design Thinking: Part II, Design and Culture, 4:2, 129-148, DOI: 10.2752/175470812X13281948975413

Knight, R. E., Shoveller, J. A., Carson, A. M. and Contreras-Whitney, J. G., (2014) Examining clinicians' experiences providing sexual health services for LGBTQ youth: considering social and structural determinants of health in clinical practice. HEALTH EDUCATION RESEARCH Vol.29 no.4 2014 Pages 662-670

Latour, B \& Woolgar, S. (1986) Laboratory Life - The Construction of Scientific Facts. Princeton University Press. $2^{\text {nd }}$ Edition.

Lavin, M. (2003) Who Gets to Say What to Whom; in Citizen Designer: Perspectives on Design Responsibility, edited by Heller, S. and Vienne, V. $1^{\text {st }}$ edition. Allworth.

Lie, I.K. (2016) 'Make Us More Useful to Society!': The Scandinavian Design Students' Organization (SDO) and Socially Responsible Design, 1967-1973, Design and Culture, 8:3, 327-361,DOI: 10.1080/17547075.2016.1223363

Liedtka, J., Salzman, R., \& Azer, D., (2017) Design Thinking for the Greater Good Innovation in the Social Sector. Columbia Business School Publishing.

Logie, C.H. and Lys, C., (2015) The process of developing a community-based research agenda with lesbian, gay, bisexual, transgender and queer youth in the Northwest Territories, Canada, International Journal of Circumpolar Health, 74:1, DOI: 10.3402/ijch.v74.28188

Logie, CH. \& Rwigema, M.J (2014) "The Normative Idea of Queer is a White Person": Understanding Perceptions of White Privilege Among Lesbian, Bisexual, and Queer Women of Color in Toronto, Canada, Journal of Lesbian Studies, 18:2, 174191, DOI: $\underline{10.1080 / 10894160.2014 .849165}$

MacLeod, M. and Macdonald, I. (2016) Developing intercultural competencies and new perspectives on graphic design pedagogy through international volunteering. In Innovations in Learning and Teaching. Merchiston Press, Edinburgh, pp. 185-197. ISBN 978-0-9576882-8-5 
Manzini E. (2007) Design Research for Sustainable Social Innovation. In: Michel R. (eds) Design Research Now. Board of International Research in Design. Birkhäuser Basel

Maritz, A., \& Laferriere, R. (2016). Entrepreneurship and self-employment for people with disabilities. Australian Journal of Career Development, 25(2), 45-

54. https://doi.org/10.1177/1038416216658044

Martin, R. (2009) The Design of Business Why Design Thinking Is the Next Competitive Advantage Harvard Business Press.

Martos, A.J. Wilson, P.A. Meyer, I.H. (2017) Lesbian, gay, bisexual, and transgender (LGBT) health services in the United States: Origins, evolution, and contemporary landscape. PLoS ONE 12(7): e0180544. https://doi.org/10.1371/journal.pone.0180544

Matthews, J, H. (2009) Relationships between entrepreneurship, creativity and design: implications for entrepreneurship theory and practice. In Australian Graduate School of Entrepreneurship 6th International Entrepreneurship Research Exchange, 3-6 February, University of Adelaide, Adelaide

Markussen, T. (2013). The Disruptive Aesthetics of Design Activism: Enacting Design Between Art and Politics. Design Issues, 29(1), 38-50. Retrieved from http://www.jstor.org.proxy.library.carleton.ca/stable/24267101

Marshall-Baker, A. (2011) Design Futuring: Sustainability, Ethics and New Practice, by Tony Fry, Interiors, 2:1,138-140, DOI: 10.2752/204191211X12980384100355

Marttila, T., (2011) Designing Anti-Activism: Apocalypse faster! Nordic Design Research

Matyók, T., Mendoza, H.R., \& Schmitz, C. L. (2014). Deep Analysis: Designing Complexity into Our Understanding of Conflict. InterAgency Journal (5)2, 14-24

Maun, I. and Myhill, D. (2005), Text as design, writers as designers. English in Education, 39: 5-21. doi:10.1111/j.1754-8845.2005.tb00614.x

Mcnamara, T. and Descubes, I. (2016) Targeted Marketing to the Lesbian, Gay, Bisexual and Transgender (LGBT) Community: A primer. Rennes School of Business. DOI: 10.13140/RG.2.1.3957.6728 The ESC

Mendoza, H. R. and Matyók, T. (2013) Designing Student Citizenship: Internationalized Education in Transformative Disciplines. International Journal of Art \& Design Education, $32,215-225$ 
McRuer, R. (2005). Crip Eye for the Normative Guy: Queer Theory and the Disciplining of Disability Studies. PMLA, 120(2), 586-592. Retrieved from http://www.jstor.org/stable/25486189

McRuer, R. (2006). Crip Theory. Cultural Signs of Queerness and Disability. New York University Press.

McWilliams, J. (2016) Queering Participatory Design Research, Cognition and Instruction, 34:3, 259-274, DOI: 10.1080/07370008.2016.1172436

Meyer I. H. (2016). The Elusive Promise of LGBT Equality. American journal of public health, 106(8), 1356-8.

Middaugh, E., Bowyer, B., \& Kahne, J. (2017). U Suk! Participatory Media and Youth Experiences with Political Discourse. Youth \& Society, 49(7), 902-922.

https://doi.org/10.1177/0044118X16655246

Miller, R. A. (2017). "My Voice Is Definitely Strongest in Online Communities": Students Using Social Media for Queer and Disability Identity-Making. Journal of College Student Development 58(4), 509-525. Johns Hopkins University Press.

Mitchell, C., De Lange, N., \& Nguyen, X. T. (2016). The participation of girls with disabilities in Vietnam in a photovoice project. In J. Coffey, S. Budgeon, \& H. Cahill (Eds). Learning bodies- the body in youth and childhood studies (pp. 241-257). Singapore:

Springer

Mulé, N. J., Ross, L. E., Deeprose, B., Jackson, B. E., Daley, A., Travers, A., \& Moore, D. (2009). Promoting LGBT health and wellbeing through inclusive policy development. International Journal for Equity in Health, 8, 18. http://doi.org/10.1186/1475-9276-8-18

Mulé, N. J. and Smith, M. (2014), Invisible Populations. Can Public Admin, 57: 234-255. doi: $10.1111 /$ capa.12066

Mulgan, G. (2006). The process of social innovation. Innovations: Technology, Governance, Globalization, 1, 145-162

Muñoz, J.E., (2009) Cruising Utopia: The Then and There of Queer Futurity. NYU Press.

Murray, J. B., Evers, D. J., \& Janda, S. (1995). Marketing, Theory Borrowing, and Critical Reflection. Journal of Macromarketing, 15(2), 92-

106. https://doi.org/10.1177/027614679501500207 
Nandan, M, London, M \& Bent-Goodley, T. (2015) Social Workers as Social Change Agents: Social Innovation, Social Intrapreneurship, and Social Entrepreneurship, Human

Service Organizations: Management, Leadership \& Governance, 39:1, 38-

56, DOI: $10.1080 / 23303131.2014 .955236$

Nicholls, A. \& Murdock, A. (2012) Social Innovation Blurring Boundaries to Reconfigure Markets. New York: Pulgrave Macmillan.

Northridge, M.E., Mcgrath, B.P., Quan Krueger, S. (2007) Using Community-Based Participatory Research to Understand and Eliminate Social Disparities in Health for Lesbian, Gay, Bisexual, and Transgender Populations. The Health of Sexual Minorities. DOI: 10.1007/978-0-387-31334-4_18

Nielsen, L.M, Wikeström, A., and Tollestrup, C. (2013) Design based entrepreneurship, International Conference on Engineering Design, ICED13, Sungyunkwan University, Seoul, Korea.

Ontario Human Right Commission (1996) [Revised in 2008] Policy on HIV/AIDS related discrimination. Retrieved from: http://www.ohrc.on.ca/sites/default/files/policy\%20on\%20hiv\%20aids\%20related\%20di scrimination.pdf

Papanek, V. (1971) Design for the Real World. Human Ecology and Social Change. Academy Chicago Publisher.

Papanek, V. (1984) Design for Human Scale. Van Nostrand Reinhold Company.

PFK Consulting. (2009) Ontario Major Festivals and Events Attraction Research Study. Ministry of Tourism. Retrieved

from: http://www.mtc.gov.on.ca/en/publications/Ontario Major Festivals and Events Attraction.pdf

Perceptive Analytics (2019) Exploratory Factor Analysis in R. Contributed by Modi,M., Sai Ram, P., Veetil, S. and Sagar, C. Retrieved from: https://www.r-

bloggers.com/exploratory-factor-analysis-in-r/

Phillips, W., Lee, H., Ghobadian, A., O’Regan, N., \& James, P. (2015). Social innovation and social entrepreneurship: A systematic review. Group \& Organization Management, 40(3), 428-461.

Piepzna-Samarasinha, L. L. (2018) Care Work: Dreaming Disability Justice. Arsenal Pulp Press 
Pol, E., \& Ville, S. (2009). Social innovation: Buzz word or enduring term. Journal of Socioeconomics, $38,878-885$

Porch, W. and Yates, T. C (2018) Charting the Course: Exploring HIV, Employment and Income Security through an Episodic Disability Lens.pg.181 to 191. In, Seeing Red: HIV AIDS and Public Policy in Canada. edited by Hindmarch, S., Orsini, M., and Gagnon, M. (2018) University of Toronto Press.

Prevention Access Campaign. (2018) Consensus Statement. Retrieved from https://www.preventionaccess.org/consensus

Raley, BA \& J Lucas, J.L (2006) Stereotype or Success?, Journal of Homosexuality, 51:2, 19-38, DOI: 10.1300/J082v51n02 02

Rezai, M. \& Khazaei, M. (2017) THE CHALLENGE OF BEING ACTIVIST-DESIGNER. An attempt to understand the New Role of Designer in the Social change based on current experiences, The Design Journal, 20:sup1, S3516-S3535, DOI:

10.1080/14606925.2017.1352855

Riger S. (2002) What's Wrong with Empowerment. In Revenson, T.A. et al. (eds) A Quarter Century of Community Psychology. Springer, Boston, MA

RHO (2012) COMMUNITY-BASED RESEARCH WITH LGBTQ COMMUNITIES. Contributed by Rainbow Health Ontario's Travers, R. Retrieved from:

https://www.rainbowhealthontario.ca/wpcontent/uploads/woocommerce uploads/2012/10/COMMUNITYBASEDRESEARCHWITHLGBTCOMMUNITIES E.pdf

Saewyc, E. M., Konishi, C., Rose, H. A., \& Homma, Y. (2014). School-Based Strategies to Reduce Suicidal Ideation, Suicide Attempts, and Discrimination among Sexual Minority and Heterosexual Adolescents in Western Canada. International journal of child, youth \& family studies: IJCYFS, 5(1), 89-

112. DOI: $\underline{\text { https://doi.org/10.18357/ijcyfs.saewyce.512014 }}$

Sanders, E.B.N \& Stappers, P.J., (2008) Co-creation and the new landscapes of design, CoDesign, 4:1, 5-18, DOI: 10.1080/15710880701875068

Shaheen, G, E. (2016) “Inclusive Entrepreneurship": A Process for Improving SelfEmployment for People with Disabilities, Journal of Policy Practice, 15:1-2, 5881, DOI: $10.1080 / 15588742.2016 .1109963$

Schultz, T., Abdulla, D., Ansari, A., Canlı E., Keshavarz, M., Kiem, M., Prado de O. Martins, L. \& Vieira de Oliveira, P.J.S. (2018) What Is at Stake with Decolonizing Design? A 
Roundtable, Design and Culture, 10:1, 81-101, DOI: $\underline{10.1080 / 17547075.2018 .1434368}$

Shea, A. 2012. Designing for Social Change, strategies for community-based graphic design. New York, New York: Princeton Architectural Press.

Selloni, D. \& Corubolo, M. (2017) Design for Social Enterprises: How Design Thinking Can Support Social Innovation within Social Enterprises, The Design Journal, 20:6, 775 794, DOI: $\underline{10.1080 / 14606925.2017 .1372931}$

Shanon K. Phelan, Virginia Wright \& Barbara E. Gibson (2014) Representations of disability and normality in rehabilitation technology promotional materials, Disability and Rehabilitation, 36:24, 2072-2079, DOI: 10.3109/09638288.2014.891055

Verganti, R. (2009) Design Driven Innovation: Changing the Rules of Competition by Radically Innovating What Things Mean. Harvard Business Review Press.

Vick, A. (2012). THEORIZING EPISODIC DISABILITIES: The Case for an Embodied Politics. Canadian Social Work Review / Revue Canadienne De Service Social, 29(1), 4160. Retrieved from http://www.jstor.org/stable/43486268

Tiwari, M. (2017) Exploring the Role of the Capability Approach in Social Innovation, Journal of Human Development and Capabilities, 18:2, 181196, DOI: $\underline{10.1080 / 19452829.2016 .1271312}$

Thorpe, A. (2011). Defining design as activism, Retrieved from https://designactivism.net/wp-content/uploads/2011/05/Thorpedefiningdesignactivism.pdf

Tsai, W. H.S (2004) Gay Advertising as Negotiations: Representations of Homosexual, Bisexual and Transgender People in Mainstream Commercials. - Gender and Consumer Behavior Volume 7, Association for Consumer Research.

Vagias, W. M. (2006). "Likert-type scale response anchors. Clemson International Institute for Tourism \& Research Development, Department of Parks, Recreation and Tourism Management. Clemson University

von Jacobi, N., Edmiston, D. \& Ziegler, R. (2017) Tackling Marginalization through Social Innovation? Examining the EU Social Innovation Policy Agenda from a Capabilities Perspective, Journal of Human Development and Capabilities, 18:2, 148-

162,DOI: $10.1080 / 19452829.2016 .1256277$

von Jacobi, N., Nicholls, A. \& Chiappero-Martinetti, E. (2017) Theorizing Social Innovation to Address Marginalization, Journal of Social Entrepreneurship, 8:3, 265270, DOI: $\underline{10.1080 / 19420676.2017 .1380340}$ 
Warner, D.N. (2004) Towards a queer research methodology, Qualitative Research in Psychology,1:4, 321-337, DOI: 10.1191/1478088704qp021oa

Webster, J.R., Adams, G.A, Maranto, C.L., Sawyer, K., Thoroughgood C. (2018) Workplace contextual supports for LGBT employees: A review, meta-analysis, and agenda for future research. Hum Resour Manage. ; 57:193210. https://doi.org/10.1002/hrm.21873

Willis, P. (2010) Laboring in Silence: Young Lesbian, Gay, Bisexual, and Queer-Identifying Workers' Negotiations of the Workplace Closet in Australian Organizations. Youth \& Society. Vol 43, Issue 3, pp. 957981 https://doi.org/10.1177/0044118X10377650

Bryan-Wilson, J. (2015) Gay Semiotics Revisited. Aperture.org Retrieved from: https://aperture.org/blog/gay-semiotics-revisited/

Winterbauer, N. L., Bekemeier, B., VanRaemdonck, L., \& Hoover, A. G. (2016). Applying Community-Based Participatory Research Partnership Principles to Public Health Practice-Based Research Networks. SAGE Open. https://doi.org/10.1177/2158244016679211

Witeck Communications (2013) America's LGBT 2013 Buying Power Estimated at $\$ 830$ Billion Retrieved from http://www.witeck.com/pressreleases/lgbt-2013-buying-power/

Witter-Merithew (n.d) Retrieved from: https://www.unco.edu/cebs/aslinterpreting/pdf/library/content-mapping.pdf

Yin, L. Fassi, D. Cheng, H. Han, H. \& He, H. (2017) Health Co-Creation in Social Innovation: Design Service for Health-Empowered Society in China, The Design Journal, 20:sup1,S2293-S2303, DOI: 10.1080/14606925.2017.1352745

Young, S. and TEDxSydney. (2014) I am not your inspiration, thank you very much. Retrieved

from: https://www.ted.com/talks/stella young i m not your inspiration thank you very much

Zajzon, N., Bohemia, E., and Prendville, S., (2017). Exploring articulations of design activism. IN: Bohemia, E., de Bont, C. and Holm, L.S. (eds.) Conference Proceedings of the Design Management Academy 2017: Research Perspectives on Creative Intersections, Hong Kong, 7-9th June 2017. London: Design Management Academy, vol. 4, pp. 843-864. 